\title{
HOMOGENEOUS CHAOS, p-FORMS, SCALING AND THE FEYNMAN INTEGRAL
}

\author{
G. W. JOHNSON AND G. KALLIANPUR \\ Dedicated to the memory of Robert H. Cameron
}

\begin{abstract}
In a largely heuristic but fascinating recent paper, Hu and Meyer have given a "formula" for the Feynman integral of a random variable $f$ on Wiener space in terms of the expansion of $f$ in Wiener chaos. The surprising properties of scaling in Wiener space make the problem of rigorously connecting this formula with the usual definition of the analytic Feynman integral a subtle one. One of the main tools in carrying this out is our definition of the "natural extension' of $p$ th homogeneous chaos in terms of the 'scale-invariant lifting' of $p$-forms on the white noise space $L^{2}\left(\mathbb{R}_{+}\right)$connected with Wiener space. The key result in our development says that if $f_{p}$ is a symmetric function in $L^{2}\left(\mathbb{R}_{+}^{p}\right)$ and $\psi_{p}\left(f_{p}\right)$ is the associated $p$-form on $L^{2}\left(\mathbb{R}_{+}\right)$, then $\psi_{p}\left(f_{p}\right)$ has a scaled $L^{2}$-lifting if and only if the ' $k$ th limiting trace' of $f_{p}$ exists for $k=0,1, \ldots,[p / 2]$. This necessary and sufficient condition for the lifting of a $p$-form on white noise space to a random variable on Wiener space is a worthwhile contribution to white noise theory apart from any connection with the Feynman integral since $p$-forms play a role in white noise calculus analogous to the role played by $p$ th homogeneous chaos in Wiener calculus.

Various $k$-traces arise naturally in this subject; we study some of their properties and relationships. The limiting $k$-trace plays the most essential role for us.
\end{abstract}

\section{INTRODUCTION}

Interesting questions concerning homogeneous chaos, scaling, $k$-traces, and the Feynman integral have been brought to light in a recent largely heuristic but fascinating paper of $\mathrm{Hu}$ and Meyer [5]. Our purpose here is to indicate a way of resolving these questions as well as several others which have arisen in the course of our research.

Let $\mathbb{R}_{+}$denote the nonnegative real numbers and let $\mathscr{C}_{0}=\mathscr{C}_{0}\left(\mathbb{R}_{+}\right)$be the space of continuous functions $x$ on $\mathbb{R}_{+}$such that $x(0)=0 . P_{1}$ will denote the standard Wiener measure on $\mathscr{C}_{0}\left(\mathbb{R}_{+}\right)$. Every $f \in L^{2}\left(\mathscr{C}_{0}\left(\mathbb{R}_{+}\right), P_{1}\right)$ has an

Received by the editors May 15, 1990 and, in revised form, June 8, 1991 .

1991 Mathematics Subject Classification. Primary 60G15, 60H05, 60H30, 60J65; Secondary 28C20, 81Q30, 49C99.

Key words and phrases. Homogeneous chaos, multiple Wiener-Itô integral, scaling on Wiener space, $p$-forms on Hilbert space, liftings, scale invariant liftings, scale invariant $\mathscr{L}^{2}$ liftings, $k$ trace, limiting $k$-trace, iterated $k$-trace, white noise, canonical Gauss measure, accessible random variables, natural extension of a multiple Wiener-Itô integral, Feynman integral, abstract Wiener space. 
expansion in Wiener chaos

$$
f=\sum_{p \geq 0} \frac{1}{p !} I_{p}\left(f_{p}\right)
$$

where $f_{p} \in L_{s}^{2}\left(\mathbb{R}_{+}^{p}\right)$, the symmetric functions which are square integrable over $\mathbb{R}_{+}^{p}$, and where $I_{p}$ denotes the $p$-fold multiple Wiener-Itô integral.

$\mathrm{Hu}$ and Meyer offer the following "formula" in terms of the expansion (1.1),

$$
E_{\sigma}(f)=\sum_{k} \frac{\left(\sigma^{2}-1\right)^{k}}{2^{k} k !} \operatorname{Tr}^{k}\left(f_{2 k}\right) .
$$

The formula (1.2) is to give the "Feynman integral" of the random variable $f$ when $\sigma^{2}$ is purely imaginary and when the right-hand side of (1.2) makes sense.

The first problem coming from the Hu-Meyer paper is that of giving a rigorous treatment of the $k$-trace, $\operatorname{Tr}^{k} f_{p}$, of $f_{p}$ where $k=0,1, \ldots,[p / 2]$ and $[p / 2]$ denotes the greatest integer in $p / 2$. We will do this in $\S \S 3$ and 9 , but, for the purpose of this introduction, the reader may think of $\operatorname{Tr}^{k} f_{p}$ as given by the (oversimplified) formula

$$
\begin{aligned}
& \left(\operatorname{Tr}^{k} f_{p}\right)\left(s_{2 k+1}, \ldots, s_{p}\right) \\
& \quad=\int_{\mathbb{R}_{+}^{k}} f_{p}\left(s_{1}, s_{1}, \ldots, s_{k}, s_{k}, s_{2 k+1}, \ldots, s_{p}\right) d s_{1} \cdots d s_{k} .
\end{aligned}
$$

The difficulties with (1.3) are clear since $f_{p}$ is defined only up to sets of Lebesgue measure 0 .

One would like to connect formula (1.2) with the usual notion of the scalarvalued analytic Feynman integral of $f$ obtained by starting with the Wiener integral

$$
\int_{\mathscr{C}_{0}} f(\sigma x) d P_{1}(x)
$$

for $\sigma>0$ and analytically continuing to $\sigma^{2}$ purely imaginary. Comparing (1.1) and (1.2), the naive hope would be that for $\sigma>0$,

$$
\int_{\mathscr{C}_{0}} \frac{1}{p !} I_{p}\left(f_{p}\right)(\sigma x) d P_{1}(x)= \begin{cases}\frac{\left(\sigma^{2}-1\right)^{k}}{2^{k} k !} \operatorname{Tr}^{k}\left(f_{2 k}\right) & \text { if } p=2 k \text { is even } \\ 0 & \text { if } p \text { is odd }\end{cases}
$$

However, (1.5) is too naive since, for $\sigma \neq 1, I_{p}\left(f_{p}\right)(\sigma x)$ is defined only on a set of $P_{1}$-measure 0 . (More will be said about this in $\S 2 \mathrm{~A}$.) In order to obtain a correct version of (1.5), the function $I_{p}\left(f_{p}\right)$ needs to be extended. Perhaps the first thing that comes to mind is to replace $I_{p}\left(f_{p}\right)$ in (1.5) with $I_{p}^{\sigma}\left(f_{p}\right)$ where $I_{p}^{\sigma}\left(f_{p}\right)$ is the Wiener-Itô integral corresponding to the variance parameter $\sigma^{2}$. This does not produce the desired result however because, even though the integral makes sense, we obtain

$$
\int_{\mathscr{C}_{0}} \frac{1}{p !} I_{p}^{\sigma}\left(f_{p}\right)(\sigma x) d P_{1}(x)=0
$$


We will show in this paper how to define $N\left[I_{p}\left(f_{p}\right)\right]$ which we will call the natural extension of the random variable $I_{p}\left(f_{p}\right)$, and we will obtain the desired formula

$$
\begin{aligned}
\int_{\mathscr{C}_{0}} \frac{1}{p !} N\left[I_{p}\left(f_{p}\right)\right](\sigma x) d P_{1}(x) \\
\quad= \begin{cases}\frac{\left(\sigma^{2}-1\right)^{k}}{2^{k} k !} \overrightarrow{\operatorname{Tr}}^{k}\left(f_{2 k}\right) & \text { if } p=2 k \text { is even, } \\
0 & \text { if } p \text { is odd. }\end{cases}
\end{aligned}
$$

$N\left[I_{p}\left(f_{p}\right)\right]$ will be defined in terms of the "scale-invariant $\mathscr{L}^{2}$-lifting" (to be defined in $\S 2 \mathrm{C})$ to random variables on $\mathscr{C}_{0}\left(\mathbb{R}_{+}\right)$of certain $(p-2 k)$-forms $(k=$ $0,1, \ldots,[p / 2])$ on the Hilbert space $L^{2}\left(\mathbb{R}_{+}\right)$.

$\mathrm{Hu}$ and Meyer have made the suggestion that in studying the problem of extension of $I_{p}\left(f_{p}\right)$ it might be more natural and basic to start not with Wiener space but with the Cameron-Martin space $\mathscr{H}$ on which the $p$ th order "multiple Wiener integral" is nothing but a homogeneous $p$-linear form $\psi_{p}$ defined on $\mathscr{H}$. Since $P_{\sigma}(\mathscr{H})=0$ for all $\sigma>0, \psi_{p}$ is obviously not a random variable in the usual sense. A theory of "accessible" random variables on a Hilbert space regarded as a finitely additive Gauss probability space has been developed and applied to problems of nonlinear prediction and filtering theory in the recent book by Kallianpur and Karandikar [13]. It turns out that this theory is the appropriate setting for the development of $\mathrm{Hu}$ and Meyer's ideas. A key concept is the notion of a lifting map to a suitable representation space, an idea that goes back to I. E. Segal (see the references in [13]). These questions will be discussed somewhat in $\S 2 \mathrm{C}$.

We remark that by taking a different choice of representation space, for example, an abstract Wiener space or the white noise space $\left(\mathscr{S}^{\prime}, \mu\right)$ where $\mu$ is the countably additive Gaussian white noise measure on the space $\mathscr{S}^{\prime}\left(\mathbb{R}^{d}\right)$ of Schwarz distributions on $\mathbb{R}^{d}$, one can obtain extensions of our main results to these spaces. Brief comments on the extension to abstract Wiener space are given in $\S 10$.

Theorem 5.1 is a key result in our development. It asserts that the $p$-form $\psi_{p}\left(f_{p}\right)$ on $L^{2}\left(\mathbb{R}_{+}\right)$associated with $f_{p} \in L_{s}^{2}\left(\mathbb{R}_{+}^{p}\right)$ has a scale-invariant $\mathscr{L}^{2}$ lifting if and only if the limiting trace, $\overrightarrow{\operatorname{Tr}}^{k} f_{p}$, exists for $k=0,1, \ldots,[p / 2]$. Further, it expresses this lifting as a finite sum of multiple Wiener-Itô integrals. The fact that the trace conditions are shown to be necessary as well as sufficient is connected with the definition of the limiting trace, $\overrightarrow{\operatorname{Tr}}^{k} f_{p}$ (see $\S 3$ ). We were led to Theorem 5.1 largely by questions that were raised in [5]. However, this theorem stands on its own as a worthwhile contribution to the white noise theory developed in [13]. Further comments related to this point can be found in $\S 5$ below.

The Feynman integral provided the initial motivation for the present work, and it, in conjunction with this paper and the paper of $\mathrm{Hu}$ and Meyer [5], suggests several further questions. However, the discussion of the Feynman integral below is limited to issues closely related to those already raised in this introduction.

We finish this introduction by outlining the contents of the paper. Section 2 deals with preliminaries most of which are known or essentially known. Section 
3 is crucial; here the limiting $k$-trace, $\overrightarrow{\operatorname{Tr}}^{k} f_{p}$, is introduced and studied. Section 4 contains two lemmas which give the results of $\S \S 5$ and 6 in the special case where $f_{p} \in L_{s}^{2}\left(\mathbb{R}_{+}^{p}\right)$ has a finite expansion in terms of a tensorial Hilbert basis $\left(\phi_{i_{1}} \otimes \cdots \otimes \phi_{i_{p}}\right)$ for $L^{2}\left(\mathbb{R}_{+}^{p}\right)$

The key result, Theorem 5.1, already mentioned above, which expresses the lifting of a $p$-form on $L^{2}\left(\mathbb{R}_{+}\right)$as a finite sum of multiple Wiener-Itô integrals or, alternatively, as a $p$-form on Wiener space, is given in $\S 5$. The rest of $\S 5$ as well as $\S \S 6$ through 8 present various consequences of Theorem 5.1. Except for $\S 8$, these results are of interest apart from any connection with the Feynman integral. In $\S 6$, the point of view of Theorem 5.1 is reversed and multiple Wiener-Itô integrals are written as finite sums of liftings of $p$-forms on $L^{2}\left(\mathbb{R}_{+}\right)$.

The natural extension $N\left[I_{p}\left(f_{p}\right)\right]$ of $I_{p}\left(f_{p}\right)$ is defined in $\S 7$ and we show how to write $N\left[I_{p}\left(f_{p}\right)\right]$ as a finite sum of multiple Wiener-Itô integrals. At this point it is easy to rigorously establish the connection between formula (1.2) of $\mathrm{Hu}$ and Meyer and the usual definition of the scalar-valued analytic Feynman integral. This is carried out in $\S 8$.

Section 9 is devoted to a rather detailed study of the various types of traces that occur naturally in the context of these problems. The evidence seems to be that the concept of " $k$-trace" will be of continuing interest; the results of $\S \S 3$ and 9 contribute to the understanding of these somewhat elusive objects.

During the course of writing this paper, the work of H. Sugita [19] was brought to our attention by S. Watanabe. Sugita's work and this paper have rather different goals but, among the concerns of [19], are questions similar to the results of $\S 10$ of our paper.

\section{PRELIMINARIES}

A. Scaling in Wiener space. A rather detailed treatment of this topic and its relationship with the Feynman integral and other matters as well as references to the earlier literature can be found in the paper [9] of the first author and Skoug. Here we need the basic facts in the setting of Wiener space on the infinite interval $\mathbb{R}_{+}$. This is the setting of the paper of Hu and Meyer and we follow their discussion for a while.

Given any $\sigma>0$, let

$$
\Omega_{\sigma}:=\left\{x \in \mathscr{C}_{0}:[x, x]_{t}=\sigma^{2} t \text { for all dyadic } t, t>0\right\}
$$

where

$$
[x, x]_{t}:=\lim _{n} \sum_{k=0}^{2^{n}-1}\left[x\left(\frac{(k+1) t}{2^{n}}\right)-x\left(\frac{k t}{2^{n}}\right)\right]^{2} .
$$

It is known $[5,9]$ that Wiener measure $P_{1}$ on $\mathscr{C}_{0}\left(\mathbb{R}_{+}\right)$is carried by $\Omega_{1}$ and that the scaled measure $P_{\sigma}:=P_{1} \circ \sigma^{-1}$ corresponding to the Wiener process with variance parameter $\sigma^{2}$ is carried by $\Omega_{\sigma}$. Clearly, $\Omega_{\sigma_{1}} \cap \Omega_{\sigma_{2}}=\varnothing$ if $\sigma_{1} \neq \sigma_{2}$ and so $P_{\sigma_{1}}$ and $P_{\sigma_{2}}$ are mutually singular. Note: When we say that $P_{\sigma}$ is carried by $\Omega_{\sigma}$ we mean that $P_{\sigma}\left(\Omega_{\sigma}\right)=1$ and not that $\Omega_{\sigma}$ is the topological support of $P_{\sigma}$; indeed, the topological support of $P_{\sigma}$ is $\mathscr{C}_{0}$ for each $\sigma>0$.

Clearly a function $F$ is defined $P_{\sigma}$-almost surely $\left(P_{\sigma}\right.$-a.s. $)$ on $\mathscr{C}_{0}$ if and only if it is defined $P_{\sigma}$-a.s. on $\Omega_{\sigma}$. Since $\Omega_{\sigma}=\sigma \Omega_{1}$ and $P_{\sigma}=P_{1} \circ \sigma^{-1}, F$ is defined $P_{\sigma}$-a.s. if and only if $F \circ \sigma$ is defined $P_{1}$-a.s. 
Next, for the convenience of the reader, we state some definitions and results that are given in [9]. Let $\mathscr{B}=\mathscr{B}\left(\mathscr{C}_{0}\left(\mathbb{R}_{+}\right)\right)$denote the $\sigma$-algebra of Borel subsets of $\mathscr{C}_{0}\left(\mathbb{R}_{+}\right)$. When $\left(\mathscr{C}_{0}, \mathscr{B}, P_{\sigma}\right)$ is completed, let $\mathscr{S}_{\sigma}$ be the resulting $\sigma$-algebra of $P_{\sigma}$-measurable sets and let $\mathscr{N}_{\sigma}$ be the collection of $P_{\sigma}$-null sets. A subset $A$ of $\mathscr{C}_{0}$ is said to be scale-invariant measurable provided that $\sigma A \in \mathscr{S}_{1}$ for all $\sigma>0$. Such a set $N$ is said to be scale-invariant null provided $P_{1}(\sigma N)=0$ for every $\sigma>0$. A property which holds except on a scale-invariant null set will be said to hold scale-invariant almost surely or s-a.s. The collection of scaleinvariant measurable (respectively, scale-invariant null) sets will be denoted $\mathscr{S}$ (resp., $\mathscr{N}$ ). It is easy to show [9, Proposition 3] that $\mathscr{S}=\bigcap_{\sigma>0} \mathscr{S}_{\sigma}$ and $\mathscr{N}=\bigcap_{\sigma>0} \mathscr{N}_{\sigma}$, and, further [9, Proposition 4], $A \in \mathscr{S}$ (resp., $A \in \mathscr{N}$ ) if and only if $A \cap \Omega_{\sigma} \in \mathscr{S}_{\sigma}$ (resp., $A \cap \Omega_{\sigma} \in \mathscr{N}_{\sigma}$ ) for every $\sigma>0$. Theorem 5 of [9] gives a simple characterization of $\mathscr{S}$ and $\mathscr{N}$ which the reader might find helpful.

A function $F: \mathscr{C}_{0}\left(\mathbb{R}_{+}\right) \rightarrow \mathbb{R}$ is said to be scale-invariant measurable provided that it is measurable with respect to the $\sigma$-algebra $\mathscr{S}$. Every Borel measurable function and so certainly every continuous function is scale-invariant measurable. Let $F: \mathscr{C}_{0} \rightarrow \mathbb{R}$ have domain $D$. It is not hard to show [9, Theorem 19] that $F$ is $s$-a.s. defined and scale-invariant measurable if and only if, for each $\sigma>0$, the restriction of $F$ to $\Omega_{\sigma}$ is $P_{\sigma}$-a.s. defined and $P_{\sigma}$-measurable. Functions $F$ and $G$ from $\mathscr{C}_{0}\left(\mathbb{R}_{+}\right)$to $\mathbb{R}$ are said to be equivalent $(F \sim G)$ if and only if they are equal $s$-a.s. This is much more refined than the usual equivalence relation which requires only that $F$ and $G$ be equal $P_{1}$-a.s. If the function $G$ is identically 0 on $\mathscr{C}_{0}$, then it is not surprising that its Feynman integral is 0 . It is possible to have a function $F$ such that $F=G P_{1}$-a.s. but the Feynman integral of $F$ fails to exist (or, alternately, exists but is not $0)$. Such examples and others from [9] show the necessity of using the refined equivalence relation in connection with the Feynman integral. On the positive side, if $G$ has an analytic Feynman integral and $F=G s$-a.s., then $F$ has the same analytic Feynman integral.

B. Multiple Wiener-Itô integrals. A discussion of this topic can be found in several places including the book of the second author [11, pp. 136-138]. We state the basic facts for the Wiener process with variance parameter $\sigma^{2}$.

Let $p$ be a positive integer. The multiple Wiener-Itô integral $I_{p}^{\sigma}(f), f \in$ $L^{2}\left(\mathbb{R}_{+}^{p}\right)$, is a random variable which is defined $P_{\sigma}$-a.s. on $\mathscr{C}_{0}\left(\mathbb{R}_{+}\right)$or, to be more precise, $P_{\sigma}$-a.s. on $\Omega_{\sigma}$. For $f, g \in L^{2}\left(\mathbb{R}_{+}^{p}\right)$ and $c \in \mathbb{R}$, we have (2.1)

$$
\begin{gathered}
I_{p}^{\sigma}(c f)=c I_{p}^{\sigma}(f) \quad\left(P_{\sigma} \text {-a.s. }\right) \\
I_{p}^{\sigma}(f+g)=I_{p}^{\sigma}(f)+I_{p}^{\sigma}(g) \quad\left(P_{\sigma} \text {-a.s. }\right)
\end{gathered}
$$

(iii) $I_{p}^{\sigma}(f)=I_{p}^{\sigma}(\tilde{f}) \quad\left(P_{\sigma}\right.$-a.s. $)$ where $\tilde{f}$ denotes the symmetrization of $f$;

$$
\begin{gathered}
I_{p}^{\sigma}(f)(\sigma x)=\sigma^{p} I_{p}^{1}(f)(x) \quad\left(P_{1} \text {-a.s. }\right) \\
E_{P_{\sigma}}\left[I_{p}^{\sigma}(f)\right]:=\int_{\mathscr{C}_{0}\left(\mathbb{R}_{+}\right)} I_{p}^{\sigma}(f)(x) d P_{\sigma}(x)=0 \\
E_{P_{\sigma}}\left[\left|I_{p}^{\sigma}(f)\right|^{2}\right]=E_{P_{\sigma}}\left[\left|I_{p}^{\sigma}(\tilde{f})\right|^{2}\right]=\sigma^{2 p} p !\|\tilde{f}\|_{2}^{2} \leq \sigma^{2 p} p !\|f\|_{2}^{2} \\
E_{P_{\sigma}}\left[I_{p}^{\sigma}(f) I_{p}^{\sigma}(g)\right]=E_{P_{\sigma}}\left[I_{p}^{\sigma}(\tilde{f}) I_{p}^{\sigma}(\tilde{g})\right]=\sigma^{2 p} p !(\tilde{f}, \tilde{g})_{L^{2}\left(\mathbb{R}_{+}^{p}\right)} .
\end{gathered}
$$


Further, if $q$ is an integer distinct from $p$ and $g \in L^{2}\left(\mathbb{R}_{+}^{q}\right)$, then (viii)

$$
E_{P_{\sigma}}\left[I_{p}^{\sigma}(f) I_{q}^{\sigma}(g)\right]=0 .
$$

Note that for $\sigma \neq 1$, one side of (iv) involves $I_{p}^{\sigma}(f)$ whereas the other does not. It is not true that $I_{p}^{1}(f)(\sigma x)=\sigma^{p} I_{p}^{1}(f)(x) \quad P_{1}$-a.s. In fact, $I_{p}^{1}(f)(\sigma x)$ is not even defined $P_{1}$-a.s. as $\sigma x \in \Omega_{\sigma} \quad P_{1}$-a.s. but $I_{p}^{1}(f)(\cdot)$ is only defined $P_{1}$-a.s. on $\Omega_{1}$.

Normalized Brownian motion, $\sigma=1$, is most often discussed by itself; then one writes $I_{p}(f)$ rather than $I_{p}^{1}(f)$. With this notation, (iv) of (2.1) asserts that for every $f \in L^{2}\left(\mathbb{R}_{+}^{p}\right)$ and $\sigma>0$,

$$
I_{p}^{\sigma}(f)(\sigma x)=\sigma^{p} I_{p}(f)(x) \quad\left(P_{1} \text {-a.s. }\right) .
$$

The collection of all the functions $I_{p}^{\sigma}(f): \Omega_{\sigma} \rightarrow \mathbb{R}$, provides an extension of $I_{p}(f)=I_{p}^{1}(f)$ from $\Omega_{1}$ to $\bigcup_{\sigma>0} \Omega_{\sigma}$. The extension is, in the language of the previous subsection, $s$-a.s. defined and scale-invariant measurable. When $p=1$, the extension just mentioned is the only one which will interest us, and we will simply use $I_{1}(f)$ to denote the extended function. In contrast, for $p \geq 2$, there is another extension of $I_{p}(f) \quad\left(=I_{p}^{1}(f)\right)$, the "natural extension $N\left[I_{p}(f)\right]$ " which will be among our main concerns. Because of this and because of its usefulness in later theorems, we will retain the $\sigma$ 's in the notation $I_{p}^{\sigma}(f)$ when $p \geq 2$.

Remark 2.1. Let $f \in L^{2}\left(\mathbb{R}_{+}\right)$. If desired, one can choose a representative in such a way that $I_{1}(f)$ is everywhere defined on $\mathscr{C}_{0}\left(\mathbb{R}_{+}\right)$and satisfies (iv) of (2.1) in the $p=1$ case for all $\sigma>0$ and $x \in \mathscr{C}_{0}\left(\mathbb{R}_{+}\right)$. Simply take $I_{1}(f)$ to be 0 off of $\bigcup_{\sigma>0} \Omega_{\sigma}$ and on those $x \in \Omega_{1}$ for which $I_{1}(f)$ is not already defined; finally, for $\sigma x \in \Omega_{\sigma}=\sigma \Omega_{1}$, take $I_{1}(f)(\sigma x)$ to be $\sigma I_{1}(f)(x)$. Formula (iv) of (2.1) shows that this everywhere defined function and the original function agree $s$-a.s. We can employ essentially the same device for $p \geq 2$ and choose a representative which is defined on all of $\mathscr{C}_{0}\left(\mathbb{R}_{+}\right)$and satisfies (iv) of (2.1) for every $\sigma>0$ and $x \in \mathscr{C}_{0}\left(\mathbb{R}_{+}\right)$.

C. Lifting and scale-invariant lifting. An extensive discussion of the concept of lifting and its applications to filtering theory along with references to the literature can be found in the book of Kallianpur and Karandikar [13]. We begin by recalling some facts connected with the canonical Gauss measure on a separable Hilbert space $H$ over $\mathbb{R}$. Let $\mathscr{P}$ denote the class of orthogonal projections on $H$ with finite dimensional range. For $\pi \in \mathscr{P}$, let

$$
\mathscr{C}_{\pi}:=\left\{\pi^{-1}(B): B \in \mathscr{B}(\pi(H)) \text {, the Borel class of the range of } \pi\right\} .
$$

$\mathscr{C}_{\pi}$ is a $\sigma$-field for each $\pi$ and $\mathscr{C}:=\bigcup_{\pi \in \mathscr{D}} \mathscr{C}_{\pi}$ is a field of subsets of $H . \mu$ will denote the finitely additive canonical Gauss measure on $H$ [13, p. 62]. $(H, \mathscr{C}, \mu)$ is called a finitely additive canonical Hilbert space.

A representation of $\mu$ is a pair $(L, P)$ where $P$ is a countably additive probability measure on some measurable space $(\Omega, \mathscr{A})$ and $L$ is a mapping (actually, an equivalence class of mappings [13, p. 81]) from $H$ into the space of $\mathbb{R}$-valued random variables on $(\Omega, \mathscr{A}, P)$ such that $L$ is linear in an appropriate sense and such that for all $C \in \mathscr{C}$,

$$
\mu(C)=P\left\{\omega \in \Omega:\left(L\left(h_{1}\right)(\omega), \ldots, L\left(h_{j}\right)(\omega)\right) \in B\right\}
$$


where $C=\left\{h \in H:\left(\left(h, h_{1}\right), \ldots,\left(h, h_{j}\right)\right) \in B\right\}$ with $h_{1}, \ldots, h_{j}$ in $H$ and $B$ a Borel subset of $\mathbb{R}^{j}$. A representation of $\mu$ always exists. In the main body of this paper, we will take $(\Omega, \mathscr{A})$ to be $\left(\mathscr{C}_{0}\left(\mathbb{R}_{+}\right), \mathscr{B}\left(\mathscr{C}_{0}\left(\mathbb{R}_{+}\right)\right)\right.$and $P$ to be one of the scaled Wiener measures $P_{\sigma}, \sigma>0$. The representation $L$ will be chosen as

$$
L(\phi)(x)=I_{1}(\phi)(x)
$$

where $\phi \in H=L^{2}\left(\mathbb{R}_{+}\right)$and $x \in \mathscr{C}_{0}\left(\mathbb{R}_{+}\right)$.

A function $f: H \rightarrow \mathbb{R}$ is a Borel cylinder function if and only if it can be written as

$$
f(h)=g\left(\left(h, h_{1}\right), \ldots,\left(h, h_{k}\right)\right)
$$

for some $k \geq 1$ and $h_{1}, \ldots, h_{k}$ in $H$ where $g: \mathbb{R}^{k} \rightarrow \mathbb{R}$ is Borel measurable. We define $R f$, the lifting of $f$, to be

$$
R(f)(\cdot):=g\left(I_{1}\left(h_{1}\right)(\cdot), \ldots, I_{1}\left(h_{k}\right)(\cdot)\right) .
$$

The discussion in $\S 2 \mathrm{~B}$ ( $p=1$ case) shows us that $R f$ is defined $s$-a.s.

Let $\sigma>0 . \mathscr{L}_{\sigma}^{0}(H, \mu)$ will denote the class of functions $f: H \rightarrow \mathbb{R}$ satisfying: For all $\pi \in \mathscr{P}$, the function $f \circ \pi(h):=f(\pi h)$ is $\mathscr{C}_{\pi}$-measurable and for all sequences $\left\{\pi_{N}\right\}$ from $\mathscr{P}$ converging strongly to the identity $\left(\pi_{N} \rightarrow I\right)$, the sequence $\left\{R\left(f \circ \pi_{N}\right)\right\}$ is Cauchy in $P_{\sigma}$-probability. One can show that all these sequences converge in $P_{\sigma}$-probability to the same limit $R_{\sigma}(f)$, called the $\sigma$-lifting of $f . R_{\sigma}(f)$ is defined $P_{\sigma}$-a.s. An $f$ in $\mathscr{L}_{\sigma}^{0}(H, \mu)$ will be called a $\sigma$-accessible random variable. The lifting usually discussed is the 1-lifting.

If $f$ has a $\sigma$-lifting for all $\sigma>0$, we let $R f=R_{\sigma} f$ on $\Omega_{\sigma}$ and we call $R f$ the scale-invariant lifting (or s-lifting) of $f$. In this case, for every $\sigma>0, R f$ is defined $P_{\sigma}$-a.s. Thus $R f$ is $s$-a.s. defined and scale-invariant measurable. A function $f$ which belongs to $\mathscr{L}_{\sigma}^{0}(H, \mu)$ for every $\sigma>0$ will be called an $s$-accessible random variable.

For any $\sigma>0$, we let $\mathscr{L}_{\sigma}^{2}(H, \mu)$ denote the set of all $f \in \mathscr{L}_{\sigma}^{0}(H, \mu)$ such that for all sequences $\left\{\pi_{N}\right\}$ from $\mathscr{P}$ with $\pi_{N} \uparrow I$,

$$
\int_{\mathscr{C}_{0}}\left|R\left(f \circ \pi_{N}\right)-R\left(f \circ \pi_{N^{\prime}}\right)\right|^{2} d P_{\sigma} \rightarrow 0
$$

as $N, N^{\prime} \rightarrow \infty$. Note that if $f \in \mathscr{L}_{\sigma}^{2}(H, \mu)$, then

$$
\int_{\mathscr{C}_{0}}\left|R_{\sigma}(f)\right|^{2} d P_{\sigma}<\infty
$$

When $f \in \mathscr{L}_{\sigma}^{2}(H, \mu)$, we call $R_{\sigma}(f)$ a $\sigma-\mathscr{L}^{2}$ lifting. If $f$ belongs to $\mathscr{L}_{\sigma}^{2}(H, \mu)$ for all $\sigma>0$, we call $R f:=R_{\sigma} f$ on $\Omega_{\sigma}$, a scale-invariant $\mathscr{L}^{2}$ lifting. If $R f$ is a scale-invariant $\mathscr{L}^{2}$-lifting then, for every $\sigma>0, R f$ is defined $P_{\sigma}$-a.s. and belongs to the space $L^{2}\left(\mathscr{C}_{0}\left(\mathbb{R}_{+}\right), P_{\sigma}\right)$ which can be identified with $L^{2}\left(\Omega_{\sigma}, P_{\sigma}\right)$.

\section{THE Limiting $k$-TRACE}

There are various ways of defining the $k$-trace of a function $f_{p} \in L_{s}^{2}\left(\mathbb{R}_{+}^{p}\right)$. In this section we focus our attention primarily on the limiting $k$-trace, $\overrightarrow{\operatorname{Tr}}^{k} f_{p}$, since it will appear in all of our principal theorems in $\S \S 5-8$. Three other 
definitions of $k$-trace will also be given. A simple case of the first of these (see Definition 3.1) will be involved in the definition of the limiting trace. The other two will be introduced in $\S 9$ where it will be shown, among other things, that for a large class of functions, all four $k$-traces exist and agree. The results of $\S 9$, while not used directly in $\S \S 3$ and $5-8$, shed light on the earlier material in various ways. For example, they provide sufficient conditions for the existence of limiting $k$-traces.

Rosinski discussed a Hilbert space valued trace in [17]. The limiting trace will be defined as the limit of certain simple cases of these traces. We give the definition from [17] just in the setting which concerns us. A somewhat more detailed discussion can be found in our earlier paper [8] and, of course, in [17].

Definition 3.1. Let $f_{p} \in L_{s}^{2}\left(\mathbb{R}_{+}^{p}\right)$ and let $0 \leq k \leq[p / 2]$ where $[p / 2]$ denotes the greatest integer in $p / 2$. We take $\operatorname{Tr}^{0} f_{p}:=f_{p}$. For $1 \leq k \leq[p / 2]$, we say that $\operatorname{Tr}^{k} f_{p}$ exists and equals $h \in L_{s}^{2}\left(\mathbb{R}_{+}^{p-2 k}\right)$ if and only if for every CONS (i.e., complete orthonormal set) $\left(e_{j}\right)$ for $L^{2}\left(\mathbb{R}_{+}^{k}\right)$,

$$
\begin{aligned}
& \sum_{j=1}^{\infty} \int_{\mathbb{R}_{+}^{k} \times \mathbb{R}_{+}^{k}} f_{p}\left(s_{1}, \ldots, s_{k} ; s_{k+1}, \ldots, s_{2 k} ; \cdot, \ldots, \cdot\right) \\
& \quad \cdot e_{j}\left(s_{1}, \ldots, s_{k}\right) e_{j}\left(s_{k+1}, \ldots, s_{2 k}\right) d s_{1} \cdots d s_{k} d s_{k+1} \cdots d s_{2 k}=h(\cdot),
\end{aligned}
$$

where the series on the left-hand side of (3.1) converges to $h$ in the norm on $L^{2}\left(\mathbb{R}_{+}^{p-2 k}\right)$.

Until $\S 9$ we will need $\operatorname{Tr}^{k} f_{p}$ as just defined only for the special functions described in our first proposition. Let $\left(\phi_{i}\right)$ be a CONS for $L^{2}\left(\mathbb{R}_{+}\right)$so that $\left\{\phi_{i_{1}} \otimes \cdots \otimes \phi_{i_{p}}: i_{l}=1,2, \ldots, l=1, \ldots, p\right\}$ is a CONS for $L^{2}\left(\mathbb{R}_{+}^{p}\right)$.

Proposition 3.1. Suppose that $f_{p} \in L_{s}^{2}\left(\mathbb{R}_{+}^{p}\right)$ has an expansion of the following form in terms of the CONS $\left\{\phi_{i_{1}} \otimes \cdots \otimes \phi_{i_{p}}\right\}$ described above

$$
f_{p}=\sum_{i_{1}, \ldots, i_{p}=1}^{N} a_{i_{1}, \ldots, i_{p}} \phi_{i_{1}} \otimes \cdots \otimes \phi_{i_{p}} .
$$

Then $\operatorname{Tr}^{k} f_{p}$ exists for every $k, 0 \leq k \leq[p / 2]$, and we have

$$
\operatorname{Tr}^{k} f_{p}=\sum_{i_{2 k+1}, \ldots, i_{p}=1}^{N}\left(\sum_{j_{1}, \ldots, j_{k}=1}^{N} a_{j_{1}, j_{1}, \ldots, j_{k}, j_{k}, i_{2 k+1}, \cdots, i_{p}}\right) \phi_{i_{2 k+1}} \otimes \cdots \otimes \phi_{i_{p}} .
$$

Proof. Let $\left(e_{j}\right)$ be any CONS for $L^{2}\left(\mathbb{R}_{+}^{k}\right)$. By Definition 3.1 it suffices to show that the series

$$
\begin{aligned}
\sum_{j=1}^{\infty} \int_{R_{+}^{k} \times R_{+}^{k}}\left[\sum_{i_{1}, \ldots, i_{p}=1}^{N} a_{i_{1}, \ldots, i_{p}} \phi_{i_{1}}\left(s_{1}\right)\right. \\
\\
\left.\quad \ldots \phi_{i_{k}}\left(s_{k}\right) \phi_{i_{k+1}}\left(s_{k+1}\right) \cdots \phi_{i_{2 k}}\left(s_{2 k}\right) \cdot \phi_{i_{2 k+1}}\left(s_{2 k+1}\right) \cdots \phi_{i_{p}}\left(s_{p}\right)\right] \\
\quad e_{j}\left(s_{1}, \ldots, s_{k}\right) e_{j}\left(s_{k+1}, \ldots, s_{2 k}\right) d s_{1} \cdots \cdot d s_{k} \cdot d s_{k+1} \cdots \cdot d s_{2 k}
\end{aligned}
$$


converges to the right-hand side of (3.3) in the norm on $L^{2}\left(\mathbb{R}_{+}^{p-2 k}\right)$. But the series in (3.4) equals

$$
\begin{aligned}
\sum_{i_{1}, \ldots, i_{2 k}=1}^{N} \sum_{j=1}^{\infty}\left(\sum_{i_{2 k+1}, \ldots, i_{p}=1}^{N} a_{i_{1}, \ldots, i_{p}} \phi_{i_{2 k+1}} \otimes \cdots \otimes \phi_{i_{p}}\right) \\
\cdot\left(\phi_{i_{i}} \otimes \cdots \otimes \phi_{i_{k}}, e_{j}\right)_{L^{2}\left(\mathbf{R}_{+}^{k}\right)} \cdot\left(\phi_{i_{k+1}} \otimes \cdots \otimes \phi_{i_{2 k}}, e_{j}\right)_{L^{2}\left(\mathbf{R}_{+}^{k}\right)} \\
=\sum_{i_{1}, \ldots, i_{2 k}=1}^{N}\left(\sum_{i_{2 k+1}, \ldots, i_{p}=1}^{N} a_{i_{1}, \ldots, i_{p}} \phi_{i_{2 k+1}} \otimes \cdots \otimes \phi_{i_{p}}\right) \\
\quad \cdot\left(\sum_{j=1}^{\infty}\left(\phi_{i_{1}} \otimes \cdots \otimes \phi_{i_{k}}, e_{j}\right)\left(\phi_{i_{k+1}} \otimes \cdots \otimes \phi_{i_{2 k}}, e_{j}\right)\right) \\
=\sum_{i_{1}, \ldots, i_{2 k}=1}^{N}\left(\sum_{i_{2 k+1}, \ldots, i_{p}=1}^{N} a_{i_{1}, \ldots, i_{p}} \phi_{i_{2 k+1}} \otimes \cdots \otimes \phi_{i_{p}}\right) \\
\quad \cdot\left(\phi_{i_{1}} \otimes \cdots \otimes \phi_{i_{k}}, \phi_{i_{k+1}} \otimes \cdots \otimes \phi_{i_{2 k}}\right) \\
=\sum_{i_{2 k+1}, \ldots, i_{p}=1}^{N}\left(\sum_{j_{1}, \ldots, j_{k}=1}^{N} a_{j_{1}, j_{1} \ldots, j_{k}, j_{k}, i_{2 k+1}, \ldots, i_{p}}\right) \phi_{i_{2 k+1}} \otimes \cdots \otimes \phi_{i_{p}}
\end{aligned}
$$

with the series in (3.5) converging in the sense of the norm $\|\cdot\|_{L^{2}\left(\mathbb{R}_{+}^{p-2 k}\right)}$.

In Theorem 9.3 we will give a result which is more general than the one just proved. However, Proposition 3.1 is all that we require at present.

Next we take advantage of Proposition 3.1 and define $\overrightarrow{\operatorname{Tr}}^{k} f_{p}$.

Definition 3.2. Let $f_{p} \in L_{s}^{2}\left(\mathbb{R}_{+}^{p}\right)$. Given any CONS $\left(\phi_{i}\right)$ for $L^{2}\left(\mathbb{R}_{+}\right)$and any positive integer $N$, let

$$
f_{p,\left(\phi_{i}\right)}^{N}=\sum_{i_{1}, \ldots, i_{p}=1}^{N} a_{i_{1}, \ldots, i_{p}} \phi_{i_{1}} \otimes \cdots \otimes \phi_{i_{p}}
$$

where $a_{i_{1}, \ldots, i_{p}}=\left(f_{p}, \phi_{i_{1}} \otimes \cdots \otimes \phi_{i_{p}}\right)$. For $k=1, \ldots,[p / 2]$, the limiting $k$-trace, $\overrightarrow{\operatorname{Tr}}^{k} f_{p}$, exists provided there is a $g \in L^{2}\left(\mathbb{R}_{+}^{p-2 k}\right)$ such that

$$
\left\|\operatorname{Tr}^{k} f_{p,\left(\phi_{i}\right)}^{N}-g\right\| \rightarrow 0 \text { as } N \rightarrow \infty
$$

for every CONS $\left(\phi_{i}\right)$ for $L^{2}\left(\mathbb{R}_{+}\right)$. The function $g$ is, by definition, $\overrightarrow{\operatorname{Tr}}^{k} f_{p}$. We take $\overrightarrow{\operatorname{Tr}}^{0} f_{p}:=f_{p}$.

It is natural to ask if $\overrightarrow{\operatorname{Tr}}^{k} f_{p}$ exists and equals $\operatorname{Tr}^{k} f_{p}$ when $f_{p}$ has a finite expansion as in Proposition 3.1 above. The answer is in the affirmative.

Proposition 3.2. Let $\left(\psi_{i}\right)$ be a CONS for $L^{2}\left(\mathbb{R}_{+}\right)$and suppose that $f_{p} \in L_{s}^{2}\left(\mathbb{R}_{+}^{p}\right)$ has the expansion

$$
f_{p}=\sum_{i_{1}, \ldots, i_{p}=1}^{M} a_{i_{1}, \ldots, i_{p}} \psi_{i_{1}} \otimes \cdots \otimes \psi_{i_{p}} .
$$

Then $\overrightarrow{\operatorname{Tr}}^{k} f_{p}$ exists and

$$
\overrightarrow{\operatorname{Tr}}^{k} f_{p}=\operatorname{Tr}^{k} f_{p}
$$


Proof. Let $\left(\phi_{i}\right)$ be any CONS for $L^{2}\left(\mathbb{R}_{+}\right)$. We must show that

$$
\left\|\operatorname{Tr}^{k} f_{p,\left(\phi_{i}\right)}^{N}-\operatorname{Tr}^{k} f_{p}\right\|_{L^{2}\left(\mathbb{R}_{+}^{p-2 k}\right)} \rightarrow 0
$$

as $N \rightarrow \infty$. Let $b_{i_{1}, \ldots, i_{p}}=\left(f_{p}, \phi_{i_{1}} \otimes \cdots \otimes \phi_{i_{p}}\right)$. To show (3.10) it suffices, by Proposition 3.1, to show that

$$
\begin{aligned}
& \| \sum_{i_{2 k+1}, \ldots, i_{p}=1}^{N}\left(\sum_{j_{1}, \ldots, j_{k}=1}^{N} b_{j_{1}, j_{1}, \ldots, j_{k}, j_{k}, i_{2 k+1}, \ldots, i_{p}}\right) \phi_{i_{2 k+1}} \otimes \cdots \otimes \phi_{i_{p}} \\
& \quad-\sum_{i_{2 k+1}, \ldots, i_{p}=1}^{M}\left(\sum_{j_{1}, \ldots, j_{k}=1}^{M} a_{j_{1}, j_{1}, \ldots, j_{k}, j_{k}, i_{2 k+1}, \ldots, i_{p}}\right) \psi_{i_{2 k+1}} \otimes \cdots \otimes \psi_{i_{p}} \| \rightarrow 0
\end{aligned}
$$

as $N \rightarrow \infty$.

From the expansion (3.8) we see that

$$
b_{i_{1}, \ldots, i_{p}}=\sum_{u_{1}, \ldots, u_{p}=1}^{M} a_{u_{1}, \ldots, u_{p}}\left(\psi_{u_{1}}, \phi_{i_{1}}\right) \cdots\left(\psi_{u_{p}}, \phi_{i_{p}}\right) .
$$

Using (3.12) to justify the first equality we can write

$$
\begin{aligned}
& \sum_{i_{2 k+1}, \ldots, i_{p}=1}^{N}\left(\sum_{j_{1}, \ldots, j_{k}=1}^{N} b_{j_{1}, j_{1}, \ldots, j_{k}, j_{k}, i_{2 k+1}, \ldots, i_{p}}\right) \phi_{i_{2 k+1}} \otimes \cdots \otimes \phi_{i_{p}} \\
& =\sum_{i_{2 k+1}, \ldots, i_{p}=1}^{N}\left\{\sum _ { j _ { 1 } , \ldots , j _ { k } = 1 } ^ { N } \left[\sum_{u_{1}, \ldots, u_{p}=1}^{M} a_{u_{1}, \ldots, u_{p}}\left(\psi_{u_{1}}, \phi_{j_{1}}\right)\left(\psi_{u_{2}}, \phi_{j_{1}}\right) \cdot \ldots\right.\right. \\
& \left.\left.\cdot\left(\psi_{u_{2 k-1}}, \phi_{j_{k}}\right) \cdot\left(\psi_{u_{2 k}}, \phi_{j_{k}}\right) \cdot\left(\psi_{u_{2 k+1}}, \phi_{i_{2 k+1}}\right) \cdots \cdot\left(\psi_{u_{p}}, \phi_{i_{p}}\right)\right]\right\} \\
& \cdot\left(\sum_{v_{2 k+1}, \ldots, v_{p}=1}^{\infty}\left(\psi_{v_{2 k+1}} \otimes \cdots \otimes \psi_{v_{p}}, \phi_{i_{2 k+1}} \otimes \cdots \otimes \phi_{i_{p}}\right) \psi_{v_{2 k+1}} \otimes \cdots \otimes \psi_{v_{p}}\right) \\
& =\sum_{v_{2 k+1}, \ldots, v_{p}=1}^{\infty} \sum_{u_{1}, \ldots, u_{p}=1}^{M} a_{u_{1}, \ldots, u_{p}}\left(\sum_{j_{1}=1}^{N}\left(\psi_{u_{1}}, \phi_{j_{1}}\right)\left(\psi_{u_{2}}, \phi_{j_{1}}\right)\right) \ldots \\
& \left(\sum_{j_{k}=1}^{N}\left(\psi_{u_{2 k-1}}, \phi_{j_{k}}\right)\left(\psi_{u_{2 k}}, \phi_{j_{k}}\right)\right)\left(\sum_{i_{2 k+1}=1}^{N}\left(\psi_{u_{2 k+1}}, \phi_{i_{2 k+1}}\right) \cdot\left(\psi_{v_{2 k+1}}, \phi_{i_{2 k+1}}\right)\right) \ldots \\
& \cdot\left(\sum_{i_{p}=1}^{N}\left(\psi_{u_{p}}, \phi_{i_{p}}\right)\left(\psi_{v_{p}}, \phi_{i_{p}}\right)\right) \psi_{v_{2 k+1}} \otimes \cdots \otimes \psi_{v_{p}} \\
& =\sum_{v_{2 k+1}, \ldots, v_{p}=1}^{\infty} \sum_{u_{1}, \ldots, u_{p}=1}^{M} a_{u_{1}, \ldots, u_{p}}\left(\psi_{u_{1}}, P_{N} \psi_{u_{2}}\right) \cdots \cdots\left(\psi_{u_{2 k-1}}, P_{N} \psi_{u_{2 k}}\right) \\
& \cdot\left(\psi_{u_{2 k+1}}, P_{N} \psi_{v_{2 k+1}}\right) \cdots \cdot\left(\psi_{u_{p}}, P_{N} \psi_{v_{p}}\right) \psi_{v_{2 k+1}} \otimes \cdots \otimes \psi_{v_{p}} \\
& =\sum_{u_{1}, \ldots, u_{p}=1}^{M} a_{u_{1}, \ldots, u_{p}}\left(\psi_{u_{1}}, P_{N} \psi_{u_{2}}\right) \\
& \cdots \cdots\left(\psi_{u_{2 k-1}}, P_{N} \psi_{u_{2 k}}\right)\left(P_{N} \psi_{u_{2 k+1}}\right) \otimes \cdots \otimes\left(P_{N} \psi_{u_{p}}\right) \text {. }
\end{aligned}
$$


We now take the limit of the last expression obtaining

$$
\begin{gathered}
\lim _{N \rightarrow \infty} \sum_{i_{2 k+1}, \ldots, i_{p}=1}^{N}\left(\sum_{j_{1}, \ldots, j_{k}=1}^{N} b_{j_{1}, j_{1}, \ldots, j_{k}, j_{k}, i_{2 k+1}, \ldots, i_{p}}\right) \phi_{i_{2 k+1}} \otimes \cdots \otimes \phi_{i_{p}} \\
=\sum_{u_{1}, \ldots, u_{p}=1}^{M} a_{u_{1}, \ldots, u_{p}}\left\{\lim _{N \rightarrow \infty}\left(\psi_{u_{1}}, P_{N} \psi_{u_{2}}\right) \cdot \ldots\right. \\
\left.\cdot\left(\psi_{u_{2 k-1}}, P_{N} \psi_{u_{2 k}}\right)\left[P_{N} \psi_{u_{2 k+1}}\right] \otimes \cdots \otimes\left[P_{N} \psi_{u_{p}}\right]\right\} \\
=\sum_{u_{1}, \ldots, u_{p}=1}^{M} a_{u_{1}, \ldots, u_{p}}\left(\psi_{u_{1}}, \psi_{u_{2}}\right) \cdots \cdots\left(\psi_{u_{2 k-1}}, \psi_{u_{2 k}}\right) \psi_{u_{2 k+1}} \otimes \cdots \otimes \psi_{u_{p}} \\
=\sum_{i_{2 k+1}, \ldots, i_{p}=1}^{M}\left(\sum_{j_{1}, \ldots, j_{k}=1}^{M} a_{j_{1}, j_{1}, \ldots, j_{k}, j_{k}, i_{2 k+1}, \ldots, i_{p}}\right) \psi_{i_{2 k+1}} \otimes \cdots \otimes \psi_{i_{p}},
\end{gathered}
$$

and so (3.12) is established and the proof is complete.

Remark 3.1. For the finite sums (3.8), the oversimplified formula (1.3) does actually give $\operatorname{Tr}^{k} f_{p}$ which equals, by Proposition 3.2, $\overrightarrow{\operatorname{Tr}}^{k} f_{p}$.

It is desirable to have theorems insuring that $\overrightarrow{\operatorname{Tr}}^{k} f_{p}$ exists for a large class of $f_{p}$ 's; Theorem 3.1 is one such result.

Theorem 3.1. Let $f_{p} \in L_{s}^{2}\left(\mathbb{R}_{+}^{p}\right)$. Suppose that there exists a CONS $\left(\psi_{i}\right)$ for $L^{2}\left(\mathbb{R}_{+}\right)$such that, in the expansion

$$
f_{p}=\sum_{i_{1}, \ldots, i_{p}=1}^{\infty} a_{i_{1}, \ldots, i_{p}} \psi_{i_{1}} \otimes \cdots \otimes \psi_{i_{p}},
$$

the coefficients $\left(a_{i_{1}}, \ldots, i_{p}\right)$ are in $l_{1}$.

Then for every $k, 0 \leq k \leq[p / 2], \overrightarrow{\operatorname{Tr}}^{k} f_{p}$ exists and is given by

(3.16) $\overrightarrow{\operatorname{Tr}}^{k} f_{p}=\sum_{i_{2 k+1}, \ldots, i_{p}=1}^{\infty}\left(\sum_{j_{1}, \ldots, j_{k}=1}^{\infty} a_{j_{1}, j_{1}, \ldots, j_{k}, j_{k}, i_{2 k+1}, \ldots, i_{p}}\right) \psi_{i_{2 k+1}} \otimes \cdots \otimes \psi_{i_{p}}$.

Though an independent proof of this result can be given it seems convenient to deduce it as a corollary (Corollary 9.11) of a similar result on the existence of $\operatorname{Tr}^{k} f_{p}$ which will be proved in $\S 9$.

The next proposition gives some information about $f_{p}$ and $\overrightarrow{\operatorname{Tr}}^{k} f_{p}$ under the assumption that $\overrightarrow{\operatorname{Tr}}^{k} f_{p}$ exists.

Proposition 3.3. Let $f_{p} \in L_{s}^{2}\left(\mathbb{R}_{+}^{p}\right)$ and suppose that $\overrightarrow{\mathrm{Tr}}^{k} f_{p}$ exists. Then for any CONS $\left(\phi_{i}\right)$ for $L^{2}\left(\mathbb{R}_{+}\right)$and associated expansion

$$
f_{p}=\sum_{i_{1}, \ldots, i_{p}=1}^{\infty} a_{i_{1}, \ldots, i_{p}} \phi_{i_{1}} \otimes \cdots \otimes \phi_{i_{p}}
$$

for $f_{p}$, we have that the limit

$$
\lim _{N \rightarrow \infty} \sum_{j_{1}, \ldots, j_{k}=1}^{N} a_{j_{1}, j_{1}, \ldots, j_{k}, j_{k}, i_{2 k+1}, \ldots, i_{p}}
$$


exists for all $i_{2 k+1}, \ldots, i_{p}$, and that

$$
\sum_{i_{2 k+1}, \ldots, i_{p}=1}^{\infty}\left(\sum_{j_{1}, \ldots, j_{k}=1}^{\infty} a_{j_{1}, j_{1}, \ldots, j_{k}, j_{k}, i_{2 k+1}, \ldots, i_{p}}\right)^{2}<\infty
$$

where the inner sum in (3.19) is taken to mean the limit in (3.18). Further, we have the formula

$$
\text { (3.20) } \overrightarrow{\operatorname{Tr}}^{k} f_{p}=\sum_{i_{2 k+1}, \ldots, i_{p}=1}^{\infty}\left(\sum_{j_{1}, \ldots, j_{k}=1}^{\infty} a_{j_{1}, j_{1}, \ldots, j_{k}, j_{k}, i_{2 k+1}, \ldots, i_{p}}\right) \phi_{i_{2 k+1}} \otimes \cdots \otimes \phi_{i_{p}},
$$

where the inner sum in (3.20) is again interpreted as the limit in (3.18) and the outer sum is the limit in the $L^{2}\left(\mathbb{R}_{+}^{p-2 k}\right)$-norm.

Proof. By Definition 3.2 and Proposition 3.1,

$$
\begin{aligned}
\mathscr{A}_{N} & :=\sum_{i_{2 k+1}, \ldots, i_{p}=1}^{N}\left(\sum_{j_{1}, \ldots, j_{k}=1}^{N} a_{j_{1}, j_{1}, \ldots, j_{k}, j_{k}, i_{2 k+1}, \ldots, i_{p}}\right) \phi_{i_{2 k+1} \otimes \cdots \otimes} \phi_{i_{p}} \\
& \rightarrow \overrightarrow{\operatorname{Tr}}^{k} f_{p}
\end{aligned}
$$

in $L^{2}\left(\mathbb{R}_{+}^{p-2 k}\right)$-norm as $N \rightarrow \infty$. Hence for fixed $i_{2 k+1}^{\prime}, \ldots, i_{p}^{\prime}$,

$$
\left(\mathscr{A}_{N}, \phi_{i_{2 k+1}^{\prime}} \otimes \cdots \otimes \phi_{i_{p}^{\prime}}\right) \rightarrow\left(\overrightarrow{\operatorname{Tr}}^{k} f_{p}, \phi_{i_{2 k+1}^{\prime}} \otimes \cdots \otimes \phi_{i_{p}^{\prime}}\right)
$$

But for $N \geq \max \left\{i_{2 k+1}^{\prime}, \ldots, i_{p}^{\prime}\right\}$,

$$
\left(\mathscr{A}_{N}, \phi_{i_{2 k+1}^{\prime}} \otimes \cdots \otimes \phi_{i_{p}^{\prime}}\right)=\sum_{j_{1}, \ldots, j_{k}=1}^{N} a_{j_{1}, j_{1}, \ldots, j_{k}, j_{k}, i_{2 k+1}^{\prime}, \ldots, i_{p}^{\prime}} .
$$

Formula (3.23) and (3.22) show that the limit in (3.18) exists and equals the coefficient of $\phi_{i_{2 k+1}^{\prime}} \otimes \cdots \otimes \phi_{i_{p}^{\prime}}$ in the expansion of $\overrightarrow{\mathrm{Tr}}^{k} f_{p}$. The conclusion (3.19) and formula (3.20) then follow immediately.

Proposition 3.3 gives conditions on the coefficients $\left(a_{i_{1}}, \ldots, i_{p}\right)$ which are necessary for the existence of $\overrightarrow{\operatorname{Tr}}^{k} f_{p}$. Are these conditions also sufficient? Let us state this in more detail. Let $\left(a_{i_{1}}, \ldots, i_{p}\right)$ be a sequence in $l_{2}$ which satisfies (3.18) and (3.19) and let $f_{p}$ be given by (3.17). Does $\overrightarrow{\operatorname{Tr}}^{k} f_{p}$ necessarily exist? The answer is "No" as the following example shows.

Let $p=2$ and take

$$
a_{i_{1}, i_{2}}= \begin{cases}\frac{(-1)^{i+1}}{i}, & \text { if } i_{1}=i_{2}=i \\ 0, & \text { if } i_{1} \neq i_{2}\end{cases}
$$

Then $\left(a_{i_{1}, i_{2}}\right) \in l^{2}$ and

$$
\lim _{N \rightarrow \infty} \sum_{j_{1}=1}^{N} a_{j_{1}, j_{1}}=\lim _{N \rightarrow \infty}\left\{1-\frac{1}{2}+\frac{1}{3}-\frac{1}{4}+\cdots+\frac{(-1)^{N+1}}{N}\right\},
$$


a limit which exists and is the sum of the alternating harmonic series. The condition (3.19) does not enter into the picture here since $p=2$. Now let

$$
f_{2}=\sum_{i_{1}, i_{2}=1}^{\infty} a_{i_{1}, i_{2}} \phi_{i_{1}} \otimes \phi_{i_{2}}=\sum_{i=1}^{\infty} \frac{(-1)^{i+1}}{i} \phi_{i} \otimes \phi_{i} .
$$

To see that $\overrightarrow{\operatorname{Tr}}^{1} f_{2}$ does not exist, take a permutation of the positive integers such that the corresponding rearrangement of the alternating harmonic series has a different sum. Let $\left(\psi_{i}\right)$ be the CONS obtained by making the associated permutation of the $\phi_{i}$ 's. Let $f_{2}=\sum_{i_{1}, i_{2}=1}^{\infty} b_{i_{1}, i_{2}} \psi_{i_{1}} \otimes \psi_{i_{2}}$ be the expansion of $f_{2}$ in terms of the CONS $\left\{\psi_{i_{1}} \otimes \psi_{i_{2}}\right\}$. In order for $\overrightarrow{\operatorname{Tr}}^{1} f_{2}$ to exist, Proposition 3.3 requires that the equality

$$
\lim _{N \rightarrow \infty} \sum_{j_{1}=1}^{N} b_{j_{1}, j_{1}}=\lim _{N \rightarrow \infty} \sum_{j_{1}=1}^{N} a_{j_{1}, j_{1}}
$$

hold. However, this equality fails since $\sum_{j_{1}=1}^{\infty} b_{j_{1}, j_{1}}$ is a rearrangement of the alternating harmonic series converging to a different number.

Taking $p=2$ simplified the discussion of the example above but, in fact, one can also find such examples with $p>2$ where the condition (3.19) does come into consideration.

Next we obtain two results which involve iterated limiting traces.

Definition 3.3. Let $f_{p} \in L_{s}^{2}\left(\mathbb{R}_{+}^{p}\right)$. If $\overrightarrow{\operatorname{Tr}}^{k} f_{p}$ exists for $k=0,1, \ldots,[p / 2]$, we say that $f_{p}$ has all of its first order limiting traces. Whenever $\overrightarrow{\operatorname{Tr}}^{k} f_{p}$ exists, it belongs to $L_{s}^{2}\left(\mathbb{R}_{+}^{p-2 k}\right)$ and, for $\nu=0,1, \ldots,[(p-2 k) / 2]$, it may possess a $\nu$-trace $\overrightarrow{\operatorname{Tr}}^{\nu}\left[\overrightarrow{\operatorname{Tr}}^{k} f_{p}\right]$. If all of these traces exist, $k=0,1, \ldots,[p / 2], \nu=$ $0,1, \ldots,[(p-2 k) / 2]$, we say that $f_{p}$ has all of its second order limiting traces. These second order (or iterated) traces are said to be consistent with the first order traces provided that

$$
\overrightarrow{\operatorname{Tr}}^{\nu}\left[\overrightarrow{\operatorname{Tr}}^{k} f_{p}\right]=\overrightarrow{\operatorname{Tr}}^{\nu+k} f_{p}, \quad k=0,1, \ldots,[p / 2], \nu=0,1, \ldots,[(p-2 k) / 2] .
$$

We can, of course, consider third and higher order traces. However, the next simple proposition assures us that we get nothing new beyond the second order.

Proposition 3.4. If $f_{p} \in L_{s}^{2}\left(\mathbb{R}_{+}^{p}\right)$ has all its first and second order limiting traces and they are consistent, then all the third order limiting traces of $f_{p}$ also exist and are consistent; that is, if $0 \leq k \leq[p / 2], 0 \leq \nu \leq[(p-2 k) / 2]$ and $0 \leq l \leq[(p-2 k-2 \nu) / 2]$, then $\overrightarrow{\operatorname{Tr}}^{l}\left\{\overrightarrow{\operatorname{Tr}}^{\nu}\left[\overrightarrow{\operatorname{Tr}}^{k} f_{p}\right]\right\}$ exists and

$$
\overrightarrow{\operatorname{Tr}}^{l}\left\{\overrightarrow{\operatorname{Tr}}^{\nu}\left[\overrightarrow{\operatorname{Tr}}^{k} f_{p}\right]\right\}=\overrightarrow{\operatorname{Tr}}^{k+\nu+l}\left(f_{p}\right) \text {. }
$$

It may well be that even the second order limiting traces yield nothing new. At any rate, the following proposition is true.

Proposition 3.5. Let $f_{p} \in L_{s}^{2}\left(\mathbb{R}_{+}^{p}\right)$. Suppose that there exists a CONS $\left(\psi_{i}\right)$ for $L^{2}\left(\mathbb{R}_{+}\right)$such that, in the expansion

$$
f_{p}=\sum_{i_{1}, \ldots, i_{p}=1}^{\infty} a_{i_{1}, \ldots, i_{p}} \psi_{i_{1}} \otimes \cdots \otimes \psi_{i_{p}},
$$

the coefficients $\left(a_{i_{1}}, \ldots, i_{p}\right)$ are in $l_{1}$. 
Then $f_{p}$ has all its first and second order limiting traces and they are consistent.

This result is not difficult to prove. However, it is contained in Corollary 9.12 and so we omit the proof here.

\section{THE CASE OF FINITE EXPANSIONS}

Two of our main results relating multiple Wiener-Itô integrals and liftings of $p$-forms will be given in this section in the special case where the function $f_{p}$ involved has a finite tensorial expansion. These results will serve as key lemmas for the next two sections where the corresponding general results will be proved.

We begin by introducing some notation which will be useful in this section as well as further on. Given $\phi_{i} \in L^{2}\left(\mathbb{R}_{+}\right)$, let $\xi_{i}=I_{1}\left(\phi_{i}\right)$. From the discussion preceding Remark 2.1, we have that for every $\sigma>0$,

$$
\xi_{i}(\sigma x)=\sigma \xi_{i}(x) \quad P_{1} \text {-a.s. }
$$

Recalling Remark 2.1 , we can even regard $\xi_{i}$ as everywhere defined on $\mathscr{C}_{0}\left(\mathbb{R}_{+}\right)$ and satisfying $\xi_{i}(\sigma x)=\sigma \xi_{i}(x)$ for all $\sigma>0$ and $x \in \mathscr{C}_{0}\left(\mathbb{R}_{+}\right)$.

Given $g \in L^{2}\left(\mathbb{R}_{+}^{p}\right)$, there is an associated $p$-form $\psi_{p}(g)$ acting on $H=$ $L^{2}\left(\mathbb{R}_{+}\right)$:

$$
\psi_{p}(g)(h)=\left(g, h^{\otimes p}\right)_{L^{2}\left(\mathbb{R}_{+}^{p}\right)}
$$

where $h^{\otimes p}\left(s_{1}, \ldots, s_{p}\right)=h\left(s_{1}\right) \cdots h\left(s_{p}\right)$. Note that $\xi_{i}=I_{1}\left(\phi_{i}\right)$ is the lifting of the 1-form (and cylinder function) $\psi_{1}\left(\phi_{i}\right)$ defined by $\psi_{1}\left(\phi_{i}\right)(h)=\left(\phi_{i}, h\right)$. (See (2.4) and (2.5) in $\S 2 . C$.)

Let $\left(\phi_{i}\right)$ be a CONS for $L^{2}\left(\mathbb{R}_{+}\right)$so that $\left(\phi_{i_{1}} \otimes \cdots \otimes \phi_{i_{p}}\right)$ is a CONS for $L^{2}\left(\mathbb{R}_{+}^{p}\right)$. We work throughout the rest of this section with an $f_{p} \in L_{s}^{2}\left(\mathbb{R}_{+}^{p}\right)$ which has a finite expansion,

$$
f_{p}=\sum_{i_{1}, \ldots, i_{p}=1}^{N} a_{i_{1}, \ldots, i_{p}} \phi_{i_{1}} \otimes \cdots \otimes \phi_{i_{p}} .
$$

It will be convenient for us to explicitly state as a lemma a simple consequence of the Itô decomposition formula. Itô's formula, (4.5) below, can be deduced from assertion (3.4) of Theorem 2.2 of [6].

Lemma 4.1. Let $f_{p} \in L_{s}^{2}\left(\mathbb{R}_{+}^{p}\right)$ have the finite expansion (4.3). Then for any $\sigma>0$,

$$
\begin{aligned}
I_{p}^{\sigma}\left(f_{p}\right)= & \sum_{i_{1}, \ldots, i_{p}=1}^{N} a_{i_{1}, \ldots, i_{p}} I_{p}^{\sigma}\left(\phi_{i_{1}} \otimes \cdots \otimes \phi_{i_{p}}\right) \\
= & \sum_{i_{1}, \ldots, i_{p}=1}^{N} a_{i_{1}, \ldots, i_{p}} I_{p-1}^{\sigma}\left(\phi_{i_{1}} \otimes \cdots \otimes \phi_{i_{p-1}}\right) \xi_{i_{p}} \\
& -\sigma^{2}(p-1) \sum_{i_{1}, \ldots, i_{p-1}=1}^{N} a_{i_{1}, i_{1}, i_{2}, \ldots, i_{p-1}} I_{p-2}^{\sigma}\left(\phi_{i_{2}} \otimes \cdots \otimes \phi_{i_{p-1}}\right) .
\end{aligned}
$$


The first equality in (4.4) is an immediate consequence of the linearity property of $I_{p}^{\sigma}$ on $L^{2}\left(\mathbb{R}_{+}^{p}\right)$. The key to the second equality is the Ito decomposition formula mentioned above:

$$
\begin{aligned}
I_{p}^{\sigma}\left(\phi_{i_{1}} \otimes \cdots \otimes \phi_{i_{p}}\right)= & I_{p-1}^{\sigma}\left(\phi_{i_{1}} \otimes \cdots \otimes \phi_{i_{p-1}}\right) \xi_{i_{p}} \\
& -\sigma^{2} \sum_{l=1}^{p-1} I_{p-2}^{\sigma}\left(\phi_{i_{1}} \otimes \cdots \otimes \tilde{\phi}_{i_{l}} \otimes \cdots \otimes \phi_{i_{p-1}}\right)\left(\phi_{i_{l}}, \phi_{i_{p}}\right)
\end{aligned}
$$

where the symbol $\sim$ indicates that the $l$ th function $\phi_{i_{l}}$ is omitted leaving a $(p-2)$-fold tensor.

We are now ready for the first of the two lemmas mentioned in the opening paragraph of this section. The formula involved appears in a paper of Balakrishnan [2, p. 26]. Balakrishnan's result is for "band-limited white noise" in the case $\sigma=1$; nevertheless, the key ideas appear in [2].

Given a positive integer $p$ and a nonnegative integer $k$ such that $0 \leq k \leq$ $[p / 2]$, let

$$
C_{p, k}:=\frac{p !}{(p-2 k) ! 2^{k} k !}
$$

These constants will appear frequently throughout the rest of the paper.

Lemma 4.2. Let $f_{p} \in L_{s}^{2}\left(\mathbb{R}_{+}^{p}\right)$ be given by the finite expansion (4.3). Then, for any $\sigma>0$, we have

$$
\begin{aligned}
I_{p}^{\sigma}\left(f_{p}\right) & =\sum_{i_{1}, \ldots, i_{p}=1}^{N} a_{i_{1}, \ldots, i_{p}} I_{p}^{\sigma}\left(\phi_{i_{1}} \otimes \cdots \otimes \phi_{i_{p}}\right) \\
& =\sum_{k=0}^{[p / 2]}(-1)^{k} \sigma^{2 k} C_{F, k} \sum_{i_{2 k+1}, \ldots, i_{p}=1}^{N} \sum_{i_{1}, \ldots, i_{k}=1}^{N} a_{i_{1}, i_{1}, \ldots, i_{k}, i_{k}, i_{2 k+1}, \ldots, i_{p}} \cdot \xi_{i_{2 k+1}} \cdots \cdots \xi_{i_{p}} \\
& =\sum_{k=0}^{[p / 2]}(-1)^{k} \sigma^{2 k} C_{p, k} R\left[\psi_{p-2 k}\left(\operatorname{Tr}^{k} f_{p}\right)\right] .
\end{aligned}
$$

Proof. The subscripts on the $a$ 's range from 1 to $N$ throughout, but we will suppress this in the notation.

The first equality in (4.7) comes simply from (4.3) and the linearity of $I_{p}^{\sigma}$ acting on $L^{2}\left(\mathbb{R}_{+}^{p}\right)$. The third equality comes from formula (2.5) for lifting cylinder functions, (4.2) above and the fact (see Propositions 3.1 and 3.2) that

$$
\operatorname{Tr}^{k} f_{p}=\sum_{i_{2 k+1}, \ldots, i_{p}} \sum_{i_{1}, \ldots, i_{k}} a_{i_{1}, i_{1}, \ldots, i_{k}, i_{k}, i_{2 k+1}, \ldots, i_{p}} \phi_{i_{2 k+1}} \otimes \cdots \otimes \phi_{i_{p}} .
$$

Our main task then is to establish the second equality in (4.7).

We proceed by induction on $p$. When $p=1$, the sum over $k$ reduces to the $k=0$ term. Further, when $k=0$, the $a$ 's do not have any repeated indices. Thus we see that when $p=1$, the third expression in (4.7) is just another way of writing the second expression. 
The case $p=2$ is readily obtained from (4.4) of Lemma 4.1:

$$
\begin{aligned}
\sum_{i_{1}, i_{2}} a_{i_{1}, i_{2}} I_{2}^{\sigma}\left(\phi_{i_{1}} \otimes \phi_{i_{2}}\right) & =\sum_{i_{1}, i_{2}} a_{i_{1}, i_{2}} I_{1}^{\sigma}\left(\phi_{i_{1}}\right) \xi_{i_{2}}-\sigma^{2} \sum_{i_{1}} a_{i_{1}, i_{1}} \\
& =\sum_{i_{1}, i_{2}} a_{i_{1}, i_{2}} \xi_{i_{1}} \xi_{i_{2}}-\sigma^{2} \sum_{i_{1}} a_{i_{1}, i_{1}}
\end{aligned}
$$

where the second equality comes from the fact that $I_{1}^{\sigma}\left(\phi_{i_{1}}\right)=\xi_{i_{1}}$ for every $\sigma>0$.

The induction step also depends on Lemma 4.1. The argument is straightforward but somewhat tedious, and we omit it.

Remark 4.1. Lemma 4.2 makes the idea of the "natural extension" of $I_{p}\left(f_{p}\right)=$ $I_{p}^{1}\left(f_{p}\right)$ rather transparent in the case of $f_{p}$ 's with finite expansions. Hu and Meyer [5] sought to extend $I_{p}\left(f_{p}\right)$ in such a way as to preserve polynomials in first order stochastic integrals. That this is a reasonable strategy in connection with the Feynman integral was quite believable to the present authors since it is consistent with their earlier work, for example [7 or 12], relating the Fresnel integral of Albeverio and Hoegh-Krohn [1] which deals with certain functions on a Hilbert space $H$ to the Fresnel (or Feynman) integral of corresponding functions on the Wiener space $[3,10]$ or abstract Wiener space [12] associated with $H$.

Applying Lemma 4.2 with $\sigma=1$, we get the formulas

$$
\begin{aligned}
I_{p}\left(f_{p}\right) & =\sum_{k=0}^{[p / 2]}(-1)^{k} C_{p, k} \sum_{i_{2 k+1}, \ldots, i_{p}=1}^{N} \sum_{i_{1}, \ldots, i_{k}=1}^{N} a_{i_{1}, i_{1}, \ldots, i_{k}, i_{k}, i_{2 k+1}, \ldots, i_{p}} \cdot \xi_{i_{2 k+1}} \cdots \cdot \xi_{i_{p}} \\
& =\sum_{k=0}^{[p / 2]}(-1)^{k} C_{p,{ }_{k}} R\left[\psi_{p-2 k}\left(\operatorname{Tr}^{k} f_{p}\right)\right] .
\end{aligned}
$$

Since the natural extension $N\left[I_{p}\left(f_{p}\right)\right]$ is to preserve polynomials in first order stochastic integrals, that is, in the $\xi_{i}$ 's, it is clear how $N\left[I_{p}\left(f_{p}\right)\right]$ should be defined in this case; it should continue to be given by either of the last two expressions in (4.9),

$$
\begin{aligned}
N\left[I_{p}\left(f_{p}\right)\right]= & \sum_{k=0}^{[p / 2]}(-1)^{k} C_{p, k} \sum_{i_{2 k+1}, \ldots, i_{p}=1}^{N} \\
& \cdot \sum_{i_{1}, \ldots, i_{k}=1}^{N} a_{i_{1}, i_{1}, \ldots, i_{k}, i_{k}, i_{2 k+1}, \ldots, i_{p}} \cdot \xi_{i_{2 k+1}} \cdots \cdot \xi_{i_{p}} \\
= & \sum_{k=0}^{[p / 2]}(-1)^{k} C_{p, k} R\left[\psi_{p-2 k}\left(\operatorname{Tr}^{k} f_{p}\right)\right] .
\end{aligned}
$$

Since each $\xi_{i}$ is $s$-a.s. defined and may even be taken as everywhere defined, the same can be said of $N\left[I_{p}\left(f_{p}\right)\right]$ in the present situation.

In the general case in $\S 7$ where infinite expansions are involved, we will need to work harder to define the natural extension. Briefly, for every $\sigma>0$, we will take limits in the space $L^{2}\left(\Omega_{\sigma}, P_{\sigma}\right)$ of polynomials in first order stochastic integrals.

In Lemma 4.2 a multiple Wiener-Itô integral $I_{p}^{\sigma}\left(f_{p}\right)$ was expressed as a sum of $(p-2 k)$-forms, $k=0,1, \ldots,[p / 2]$. Lemma 4.3 will express a $p$-form, 
restricted to $\Omega_{\sigma}$, as a sum of multiple Wiener-Itô integrals $I_{p-2 k}^{\sigma}\left(\operatorname{Tr}^{k} f_{p}\right), k=$ $0,1, \ldots,[p / 2]$. Lemma 4.2 is the key to Lemma 4.3 which will, in turn, be essential to the developments in later sections. Formla (4.13) below is essentially formula (10) in Hu and Meyer [5], but in [5], it is a remark which is not pursued.

Again $f_{p}$ will be assumed to have the finite expansion (4.3). Hence, the associated $p$-form $\psi_{p}\left(f_{p}\right)$ on $H=L^{2}\left(\mathbb{R}_{+}\right)$(see (4.2)) will be the cylinder function

$$
\psi_{p}\left(f_{p}\right)(h)=\sum_{i_{i}, \ldots, i_{p}=1}^{N} a_{i_{1}, \ldots, i_{p}}\left(\phi_{i_{1}}, h\right) \cdots\left(\phi_{i_{p}}, h\right) .
$$

Hence $\psi_{p}\left(f_{p}\right)$ certainly has a lifting,

$$
\Psi_{p}(x)=R\left[\psi_{p}\left(f_{p}\right)\right](x)=\sum_{i_{1}, \ldots, i_{p}=1}^{N} a_{i_{1}, \ldots, i_{p}} \xi_{i_{1}}(x) \cdots \xi_{i_{p}}(x),
$$

which is a $p$-form on $\mathscr{C}_{0}\left(\mathbb{R}_{+}\right)$which may be regarded as everywhere defined.

Lemma 4.3. Let $f_{p} \in L_{s}^{2}\left(\mathbb{R}_{+}^{p}\right)$ be given by the finite expansion (4.3) and let the associated p-form $\Psi_{p}=R\left[\psi_{p}\left(f_{p}\right)\right]$ be given on $\mathscr{C}_{0}\left(\mathbb{R}_{+}\right)$by (4.12). Then s-a.s.

$$
\Psi_{p}=R\left[\psi_{p}\left(f_{p}\right)\right]=\sum_{k=0}^{[p / 2]} \sigma^{2 k} C_{p, k} I_{p-2 k}^{\sigma}\left(\operatorname{Tr}^{k} f_{p}\right)
$$

Proof. We proceed by induction on $p$. The case $p=1$ is trivial and the case $p=2$ is easily obtained from Lemma 4.2. We now assume that (4.13) holds for all integers less than $p$ and examine the $p$ th case. The $k=0$ term in the third expression in (4.7) is just $\Psi_{p}$. Thus we can write

$$
\begin{aligned}
\Psi_{p}= & I_{p}^{\sigma}\left(f_{p}\right)+\sum_{\nu=1}^{[p / 2]}(-1)^{\nu+1} \sigma^{2 \nu} C_{p, \nu} \\
& \cdot \sum_{i_{2 \nu+1}, \ldots, i_{p}}\left(\sum_{i_{1}, \ldots, i_{\nu}} a_{i_{1}, i_{1}, \ldots, i_{\nu}, i_{\nu}, i_{2 \nu+1}, \ldots, i_{p}}\right) \xi_{i_{2 \nu+1}} \cdots \cdot \xi_{i_{p}} \\
= & I_{p}^{\sigma}\left(f_{p}\right)+\sum_{\nu=1}^{[p / 2]}(-1)^{\nu+1} \sigma^{2 \nu} C_{p, \nu}\left\{\sum_{i_{2 \nu+1}, \ldots, i_{p}} c_{i_{2 \nu+1}, \ldots, i_{p}} \xi_{i_{2 \nu+1}} \cdots \cdot \xi_{i_{p}}\right\}
\end{aligned}
$$

where

$$
c_{i_{2 \nu+1}, \ldots, i_{p}}=\sum_{i_{1}, \ldots, i_{\nu}} a_{i_{1}, i_{1}, \ldots, i_{\nu}, i_{\nu}, i_{2 \nu+1}, \ldots, i_{p}} .
$$

Now we can finish the proof by applying the induction hypothesis to the inner sum in the last expression in (4.14) since $\nu \geq 1$. In the process, one makes use of the equality $1 / k !=\sum(-1)^{\nu+1} / \nu ! r$ ! where the sum is over $\{(\nu, r) \in$ $\{1, \ldots, k\} \times\{0, \ldots, k\}: \nu+r=k\}$. The equality comes from applying the Binomial Theorem to $(-1+1)^{k}$.

\section{LIFTINGS OF $p$-FORMS AND WIENER-ITÔ INTEGRALS}

Let $f_{p} \in L_{s}^{2}\left(\mathbb{R}_{+}^{p}\right)$. We show in the theorem below that the $p$-form $\psi_{p}=$ $\psi_{p}\left(f_{p}\right)$ on $L^{2}\left(\mathbb{R}_{+}\right)$associated with $f_{p}($ see $(4.2))$ has a scaled $\mathscr{L}^{2}$-lifting $R\left[\psi_{p}\right]$ 
if and only if $\overrightarrow{\operatorname{Tr}}^{k} f_{p}$ exists for $k=0,1, \ldots,[p / 2]$. Further, in this case, $R\left[\psi_{p}\right]$ is given by a $p$-form on Wiener space or, alternately, by a finite sum of multiple Wiener-Itô integrals. This result is the key to $\S \S 5-8$. Our proof that the trace conditions are necessary as well as sufficient for the existence of the lifting is tied in with the nature of the limiting $k$-trace.

Not only will the theorem below allow us to give in subsequent sections a solution to the problem which originally motivated our research, but it is potentially a useful result in connection with white noise calculus [13] where $p$-forms play a role analogous to that played by $p$ th homogeneous chaos in Wiener calculus.

Theorem 5.1. Let $f_{p} \in L_{s}^{2}\left(\mathbb{R}_{+}^{p}\right)$ and let $\psi_{p}=\psi_{p}\left(f_{p}\right)$ be the associated $p$-form on $H=L^{2}\left(\mathbb{R}_{+}\right)$.

$\psi_{p}\left(f_{p}\right)$ has a scaled $\mathscr{L}^{2}$-lifting $R\left[\psi_{p}\right]$ if and only if $\overrightarrow{\operatorname{Tr}}^{k} f_{p}$ exists for $k=$ $0,1, \ldots,[p / 2]$. In this case, s-a.s. (that is, for every $\sigma>0, P_{\sigma}$-a.s. on $\Omega_{\sigma}$ ) we have

$$
\begin{aligned}
R \psi_{p} & =\sum_{i_{1}, \ldots, i_{p}=1}^{\infty} a_{i_{1}, \ldots, i_{p}} \xi_{i_{1}} \cdots \xi_{i_{p}} \\
& =\sum_{k=0}^{[p / 2]} \sigma^{2 k} C_{p, k} I_{p-2 k}^{\sigma}\left(\overrightarrow{\operatorname{Tr}}^{k} f_{p}\right)
\end{aligned}
$$

where the second expression in (5.1) is to be interpreted on each $\Omega_{\sigma}$ as the limit in the space $L^{2}\left(\Omega_{\sigma}, P_{\sigma}\right)$ of the sequence

$$
\sum_{i_{1}, \ldots, i_{p}=1}^{N} a_{i_{1}, \ldots, i_{p}} \xi_{i_{1}} \cdots \xi_{i_{p}}
$$

Remark 5.1. The third expression in (5.1) has the advantage that it is coordinate free but the disadvantage that it must be changed with each change in $\sigma$. The lifting $R \psi_{p}$ is, of course, scale-invariant measurable as well as $s$-a.s. defined.

Proof of Theorem 5.1. We begin with considerations which are relevant to both directions of the proof. Let $\left\{\Pi_{N}\right\}$ be a sequence from $\mathscr{P}$, the class of orthogonal projections with finite-dimensional range, such that $\Pi_{N} \uparrow I$ strongly and $\operatorname{dim}\left(\Pi_{N} H\right)=d_{N}$. We can then obtain a CONS $\left(\phi_{i}\right)$ in $H$ such that $\left\{\phi_{i}: i=1, \ldots, d_{N}\right\}$ is a CONS in $\Pi_{N} H, N=1,2, \ldots$. It suffices to consider the case where $d_{N}=N$. Of course, $f_{p}$ has an expansion with respect to the CONS $\left(\phi_{i_{1}} \otimes \cdots \otimes \phi_{i_{p}}\right)$ for $L^{2}\left(\mathbb{R}_{+}^{p}\right)$,

$$
f_{p}=\sum_{i_{1}, \ldots, i_{p}=1}^{\infty} a_{i_{1}, \ldots, i_{p}} \phi_{i_{1}} \otimes \cdots \otimes \phi_{i_{p}} .
$$

Now

$$
\psi_{p}(h)=\left(f_{p}, h^{\otimes p}\right)=\sum_{i_{1}, \ldots, i_{p}=1}^{\infty} a_{i_{1}, \ldots, i_{p}}\left(\phi_{i_{1}}, h\right) \cdots\left(\phi_{i_{p}}, h\right)
$$


and so

$$
\begin{aligned}
\psi_{p} \circ \Pi_{N}(h) & =\psi_{p}\left(\Pi_{N} h\right)=\sum_{i_{1}, \ldots, i_{p}=1}^{\infty} a_{i_{1}, \ldots, i_{p}}\left(\phi_{i_{1}}, \Pi_{N} h\right) \cdots\left(\phi_{i_{p}}, \Pi_{N} h\right) \\
& =\sum_{i_{1}, \ldots, i_{p}=1}^{\infty} a_{i_{1}, \ldots, i_{p}}\left(\Pi_{N} \phi_{i_{1}}, h\right) \cdots\left(\Pi_{N} \phi_{i_{p}}, h\right) \\
& =\sum_{i_{1}, \ldots, i_{p}=1}^{N} a_{i_{1}, \ldots, i_{p}}\left(\phi_{i_{1}}, h\right) \cdots\left(\phi_{i_{p}}, h\right) \\
& =\left(f_{p}^{N}, h^{\otimes p}\right),
\end{aligned}
$$

where $f_{p}^{N}\left(f_{p,\left(\phi_{i}\right)}^{N}\right.$, more fully) satisfies

$$
f_{p}^{N}=\sum_{i_{1}, \ldots, i_{p}=1}^{N} a_{i_{1}, \ldots, i_{p}} \phi_{i_{1}} \otimes \cdots \otimes \phi_{i_{p}}
$$

It is easy to see from (5.4) how to lift the cylinder function $\psi_{p} \circ \pi_{N}$ :

$$
R\left(\psi_{p} \circ \Pi_{N}\right)=\sum_{i_{1}, \ldots, i_{p}=1}^{N} a_{i_{1}, \ldots, i_{p}} \xi_{i_{1}} \cdots \xi_{i_{p}}
$$

where $\xi_{i}(x)=I_{1}\left(\phi_{i}\right)(x)$.

Applying Lemma 4.3 we see from (5.6) that for every $\sigma>0, P_{\sigma}$-a.s. on $\Omega_{\sigma}$,

$$
R\left(\psi_{p} \circ \Pi_{N}\right)=\sum_{k=0}^{[p / 2]} \sigma^{2 k} C_{p, k} I_{p-2 k}^{\sigma}\left(\operatorname{Tr}^{k} f_{p}^{N}\right)
$$

Now suppose that $\overrightarrow{\operatorname{Tr}}^{k} f_{p}$ exists for $k=0,1, \ldots,[p / 2]$. Let $\sigma>0$ be given. It suffices to show that for any sequence $\pi_{N} \uparrow I$ as above,

$$
\left\|R\left(\psi_{p} \circ \Pi_{N}\right)-\sum_{k=0}^{[p / 2]} \sigma^{2 k} C_{p, k} I_{p-2 k}^{\sigma}\left(\overrightarrow{\operatorname{Tr}}^{k} f_{p}\right)\right\|_{L^{2}\left(\Omega_{\sigma}, P_{\sigma}\right)}^{2} \rightarrow 0
$$

as $N \rightarrow \infty$. However, using (5.7) and properties of multiple Wiener-Itô integrals (see $(2.1)$ ), we can write

$$
\begin{aligned}
\left\|R\left(\psi_{p} \circ \Pi_{N}\right)-\sum_{k=0}^{[p / 2]} \sigma^{2 k} C_{p, k} I_{p-2 k}^{\sigma}\left(\overrightarrow{\operatorname{Tr}}^{k} f_{p}\right)\right\|_{L^{2}\left(\Omega_{\sigma}, P_{\sigma}\right)}^{2} \\
=\left\|\sum_{k=0}^{2} \sigma^{2 k} C_{p, k} I_{p-2 k}^{\sigma}\left(\operatorname{Tr}^{k} f_{p}^{N}-\overrightarrow{\operatorname{Tr}}^{k} f_{p}\right)\right\|_{L^{2}\left(\Omega_{\sigma}, P_{\sigma}\right)}^{2} \\
=\sum_{k=0}^{[p / 2]}\left(\sigma^{2 k} C_{p, k}\right)^{2}(p-2 k) !\left(\sigma^{p-2 k}\right)^{2}\left\|\operatorname{Tr}^{k} f_{p}^{N}-\overrightarrow{\operatorname{Tr}}^{k} f_{p}\right\|_{L^{2}\left(\mathbb{R}_{+}^{p-2 k}\right)}^{2}
\end{aligned}
$$

but this last expression converges to 0 as $N \rightarrow \infty$ by definition of $\overrightarrow{\operatorname{Tr}}^{k} f_{p}$, $k=0,1, \ldots,[p / 2]$. 
We now know that $\psi_{p}$ has a scaled $\mathscr{L}^{2}$-lifting $R \psi_{p}$ that is equal to the third expression in (5.1). Checking (5.6), we see that for every $\sigma>0$ all three functions in (5.1) are equal $P_{\sigma}$-a.s. on $\Omega_{\sigma}$.

It remains to show that if $\psi_{p}\left(f_{p}\right)$ has a scaled $\mathscr{L}^{2}$-lifting, then $\overrightarrow{\operatorname{Tr}}^{k} f_{p}$ exists for $k=0,1, \ldots,[p / 2]$. In fact, we will show that if there exists $\sigma>0$ such that $\psi_{p}\left(f_{p}\right)$ has a $\sigma-\mathscr{L}^{2}$-lifting, then $\overrightarrow{\operatorname{Tr}}^{k} f_{p}$ exists for $k=0,1, \ldots,[p / 2]$.

Let $\left(\alpha_{i}\right)$ be an arbitrary CONS for $H=L^{2}\left(\mathbb{R}_{+}\right)$and let $\Pi_{N}$ be the orthogonal projection onto $\mathrm{sp}\left[\alpha_{1}, \ldots, \alpha_{N}\right]$. By assumption, we have

$$
E_{P_{\sigma}}\left[R\left(\psi_{p} \circ \Pi_{N}\right)-R \psi_{p}\right]^{2} \rightarrow 0 \text { as } N \rightarrow \infty .
$$

It follows that

$$
E_{P_{\sigma}}\left[R\left(\psi_{p} \circ \Pi_{N}\right)-R\left(\psi_{p} \circ \Pi_{N^{\prime}}\right)\right]^{2} \rightarrow 0 \text { as } N, N^{\prime} \rightarrow \infty .
$$

But by Lemma 4.3 and (5.5)-(5.7),

$$
R\left(\psi_{p} \circ \Pi_{N}\right)-R\left(\psi_{p} \circ \Pi_{N^{\prime}}\right)=\sum_{k=0}^{[p / 2]} \sigma^{2 k} C_{p, k} I_{p-2 k}^{\sigma}\left(\operatorname{Tr}^{k} f_{p,\left(\alpha_{i}\right)}^{N}-\operatorname{Tr}^{k} f_{p,\left(\alpha_{i}\right)}^{N^{\prime}}\right)
$$

where

$$
f_{p,\left(\alpha_{i}\right)}^{M}=\sum_{i_{1}, \ldots, i_{p}=1}^{M}\left(f_{p}, \alpha_{i_{1}} \otimes \cdots \otimes \alpha_{i_{p}}\right) \alpha_{i_{1}} \otimes \cdots \otimes \alpha_{i_{p}} .
$$

Using properties of multiple Wiener-Itô integrals (see (2.1)), we have

$$
\begin{aligned}
& E_{P_{\sigma}}[\left.R\left(\psi_{p} \circ \Pi_{N}\right)-R\left(\psi_{p} \circ \Pi_{N^{\prime}}\right)\right]^{2} \\
& \quad=\sum_{k=0}^{[p / 2]}\left(\sigma^{2 k} C_{p, k}\right)^{2}(p-2 k) !\left\|\operatorname{Tr}^{k} f_{p,\left(\alpha_{i}\right)}^{N}-\operatorname{Tr}^{k} f_{p,\left(\alpha_{i}\right)}^{N^{\prime}}\right\|_{2}^{2} .
\end{aligned}
$$

From (5.14) and (5.11), we see that

$$
\left\|\operatorname{Tr}^{k} f_{p,\left(\alpha_{i}\right)}^{N}-\operatorname{Tr}^{k} f_{p,\left(\alpha_{i}\right)}^{N^{\prime}}\right\|_{2}^{2} \rightarrow 0 \quad \text { as } N, N^{\prime} \rightarrow \infty
$$

for $k=0,1, \ldots,[p / 2]$. Because of the completeness of $L^{2}\left(\mathbb{R}_{+}^{p-2 k}\right),(5.15)$ shows that, for every CONS $\left(\alpha_{i}\right), \operatorname{Tr}^{k} f_{p,\left(\alpha_{i}\right)}^{N}$ converges to an element of $L^{2}\left(\mathbb{R}_{+}^{p-2 k}\right)$. We still need to show that this limit is independent of the CONS $\left(\alpha_{i}\right)$.

Let $\left(\beta_{i}\right)$ be any other CONS for $H$. It suffices to show that

$$
\left\|\operatorname{Tr}^{k} f_{p,\left(\alpha_{i}\right)}^{N}-\operatorname{Tr}^{k} f_{p,\left(\beta_{i}\right)}^{N}\right\|_{2} \rightarrow 0 \quad \text { as } N \rightarrow \infty .
$$

However,

$$
\begin{aligned}
R\left(\psi_{p} \circ \Pi_{N,\left(\alpha_{i}\right)}\right)-R\left(\psi_{p} \circ \Pi_{N,\left(\beta_{i}\right)}\right) & \\
= & \sum_{k=0}^{[p / 2]} \sigma^{2 k} C_{p, k} I_{p-2 k}^{\sigma}\left(\operatorname{Tr}^{k} f_{p,\left(\alpha_{i}\right)}^{N}-\operatorname{Tr}^{k} f_{p,\left(\beta_{i}\right)}^{N}\right),
\end{aligned}
$$


and so

$$
\begin{aligned}
& \sum_{k=0}^{[p / 2]} \sigma^{2 p}\left(C_{p, k}\right)^{2}(p-2 k) !\left\|\operatorname{Tr}^{k} f_{p,\left(\alpha_{i}\right)}^{N}-\operatorname{Tr}^{k} f_{p,\left(\beta_{i}\right)}^{N}\right\|_{2}^{2} \\
& \quad=E_{P_{\sigma}}\left[\sum_{k=0}^{[p / 2]} \sigma^{2 k} C_{p, k} I_{p-2 k}^{\sigma}\left(\operatorname{Tr}^{k} f_{p,\left(\alpha_{i}\right)}^{N}-\operatorname{Tr}^{k} f_{p,\left(\beta_{i}\right)}^{N}\right)\right]^{2} \\
& =E_{P_{\sigma}}\left[R\left(\psi_{p} \circ \Pi_{N,\left(\alpha_{i}\right)}\right)-R\left(\psi_{p} \circ \Pi_{N,\left(\beta_{i}\right)}\right)\right]^{2} .
\end{aligned}
$$

But the last expression in (5.18) goes to 0 as $N \rightarrow \infty$ since

$$
E_{P_{\sigma}}\left[R\left(\psi_{p} \circ \Pi_{N,\left(\alpha_{i}\right)}\right)-R\left(\psi_{p}\right)\right]^{2} \text { and } E_{P_{\sigma}}\left[R\left(\psi_{p} \circ \Pi_{N,\left(\beta_{i}\right)}\right)-R\left(\psi_{p}\right)\right]^{2}
$$

both go to 0 as $N \rightarrow \infty$. It now follows from (5.18) that (5.16) holds for $k=0,1, \ldots,[p / 2]$ as desired.

Hence, for every $k=0,1, \ldots,[p / 2]$, there exists $g_{p-2 k}$ in $L^{2}\left(\mathbb{R}_{+}^{p-2 k}\right)$ such that for every CONS $\left(\alpha_{i}\right)$ for $H$,

$$
\left\|\operatorname{Tr}^{k} f_{p,\left(\alpha_{i}\right)}^{N}-g_{p-2 k}\right\|_{2} \rightarrow 0 \text { as } N \rightarrow \infty \text {. }
$$

Therefore $\overrightarrow{\operatorname{Tr}}^{k} f_{p}$ exists as we wished to show.

Next we present some simple consequences of Theorem 5.1. (Much of the rest of this paper will give further consequences of this theorem.) The first corollary was already noted and established in the proof above but it seems worth stating formally.

Corollary 5.1. Let $f_{p} \in L_{s}^{2}\left(\mathbb{R}_{+}^{p}\right)$ and let $\psi_{p}=\psi_{p}\left(f_{p}\right)$ be the associated p-form on $H=L^{2}\left(\mathbb{R}_{+}\right)$. If there exists $\sigma>0$ such that $\psi_{p}$ has a $\sigma-\mathscr{L}^{2}$-lifting, then $\overrightarrow{\operatorname{Tr}}^{k} f_{p}$ exists for $k=0,1, \ldots,[p / 2]$. In particular, if $\psi_{p}$ has an $\mathscr{L}^{2}$-lifting with respect to the standard Wiener measure $P=P_{1}$, then $\overrightarrow{\operatorname{Tr}}^{k} f_{p}$ exists for $k=0,1, \ldots,[p / 2]$.

Corollary 5.2. Let $f_{p}$ and $\psi_{p}$ be as in Corollary 5.1. Then $\psi_{p}$ has a scaled $\mathscr{L}^{2}$. lifting if and only if there exists $\sigma>0$ such that $\psi_{p}$ has a $\sigma-\mathscr{L}^{2}$-lifting. In particular, if $\psi_{p}$ has an $\mathscr{L}^{2}$-lifting with respect to the standard Wiener measure $P=P_{1}$, then $\psi_{p}$ has a scaled $L^{2}$-lifting.

Remark 5.2. Comparing the first two expressions in (5.1) with (5.3), one sees that the series

$$
\begin{aligned}
& \sum_{i_{1}, \ldots, i_{p}=1}^{\infty} a_{i_{1}, \ldots, i_{p}} \xi_{i_{1}} \cdots \cdots \xi_{i_{p}} \\
& \quad=\lim _{N \rightarrow \infty} \sum_{i_{1}, \ldots, i_{p}=1}^{N} a_{i_{1}, \ldots, i_{p}} \xi_{i_{1}} \cdots \xi_{i_{p}}
\end{aligned}
$$

lifts $\psi_{p}$ in a natural way.

Corollary 5.3. Let $f_{p}$ and $f_{p}^{\prime}$ be in $L_{s}^{2}\left(\mathbb{R}_{+}^{p}\right)$ with associated p-forms $\psi_{p}$ and $\psi_{p}^{\prime}$, respectively. Suppose further that there exists $\sigma_{0}>0$ such that $R\left(\psi_{p}\right)$ and $R\left(\psi_{p}^{\prime}\right)$ both exist in the $\sigma_{0}-\mathscr{L}^{2}$ sense (and hence in the scaled- $\mathscr{L}^{2}$ sense). 
Then, for every $\sigma>0$,

$$
\begin{aligned}
& \left\|R\left(\psi_{p}\right)-R\left(\psi_{p}^{\prime}\right)\right\|_{L^{2}\left(\Omega_{\sigma}, P_{\sigma}\right)}^{2} \\
& \quad=\sum_{k=0}^{[p / 2]}\left(\sigma^{2 p} C_{p, k}\right)^{2}(p-2 k) !\left\|\overrightarrow{\operatorname{Tr}}^{k} f_{p}-\overrightarrow{\operatorname{Tr}}^{k} f_{p}^{\prime}\right\|_{L^{2}\left(\mathbb{R}_{+}^{P_{p}-2 k}\right)}^{2} .
\end{aligned}
$$

In particular, if $f_{p}$ and $f_{p}^{(n)}, n=1,2, \ldots$, all belong to $L_{s}^{2}\left(\mathbb{R}_{+}^{p}\right)$ and if $\psi_{p}, \psi_{p}^{(n)}, n=1,2, \ldots$ are the associated p-forms all of which possess scaled $\mathscr{L}^{2}$-liftings $R\left(\psi_{p}\right), R\left(\psi_{p}^{(n)}\right), n=1,2, \ldots$, then

$$
\left\|R\left(\psi_{p}\right)-R\left(\psi_{p}^{(n)}\right)\right\|_{L^{2}\left(\Omega_{\sigma}, P_{\sigma}\right)}^{2} \rightarrow 0 \text { as } n \rightarrow \infty
$$

for every $\sigma>0$ if and only if

$$
\left\|\overrightarrow{\operatorname{Tr}}^{k} f_{p}^{(n)}-\overrightarrow{\operatorname{Tr}}^{k} f_{p}\right\|_{L_{s}^{2}\left(\mathbb{R}_{+}^{p-2 k}\right)} \rightarrow 0 \text { as } n \rightarrow \infty
$$

for each $k=0,1, \ldots,[p / 2]$.

Proof. This follows easily from (5.1) and properties of multiple Wiener-Itô integrals.

Remark 5.3. Often, in order to work with functions on white noise space, that is on the canonical Hilbert space, $(H, \mathscr{C}, \mu)$, one must lift them to an associated countably additive representation space. It is sometimes possible to work directly in the Hilbert space. Corollary 5.3 allows us to do that for $p$-forms on $H=L^{2}\left(\mathbb{R}_{+}^{p}\right)$; the $L^{2}\left(C_{0}\left(\mathbb{R}_{+}\right), P_{\sigma}\right)$-distance from $R\left(\psi_{p}\right)$ to $R\left(\psi_{p}^{\prime}\right)$ can be calculated within the Hilbert space.

\section{WIENER-ITÔ INTEGRALS IN TERMS OF LIFTED $P$-FORMS}

The theorem of this section will show how to write the multiple Wiener-Itô integral $I_{p}^{\sigma}\left(f_{p}\right)$ as a finite sum of lifted $(p-2 k)$-forms, $k=0,1, \ldots,[p / 2]$. The $\sigma=1$ case of this result will lead us in the next section to our definition of the natural extension $N\left[I_{p}\left(f_{p}\right)\right]$ of $I_{p}\left(f_{p}\right)$. At this point the reader may wish to review Definition 3.3 and Propositions 3.4 and 3.5.

Theorem 6.1. Let $f_{p} \in L_{s}^{2}\left(\mathbb{R}_{+}^{p}\right)$ and let $\psi_{p}=\psi_{p}\left(f_{p}\right)$ be the associated p-form on $H=L^{2}\left(\mathbb{R}_{+}\right)$. Assume that $f_{p}$ has all its first and second order traces and that they are consistent.

Then for every $\sigma>0, P_{\sigma}$-a.s. on $\Omega_{\sigma}$,

$$
\begin{aligned}
I_{p}^{\sigma}\left(f_{p}\right)= & \sum_{k=0}^{[p / 2]}(-1)^{k} \sigma^{2 k} C_{p, k} R\left[\psi_{p-2 k}\left(\overrightarrow{\operatorname{Tr}}^{k} f_{p}\right)\right] \\
= & \sum_{k=0}^{[p / 2]}(-1)^{k} \sigma^{2 k} C_{p, k} \sum_{i_{2 k+1}, \ldots, i_{p}=1}^{\infty} \\
& \cdot\left(\sum_{j_{1}, \ldots, j_{k}=1}^{\infty} a_{j_{1}, j_{1}, \ldots, j_{k}, j_{k}, i_{2 k+1}, \ldots, i_{p}}\right) \xi_{i_{2 k+1}} \cdots \cdot \xi_{i_{p}}
\end{aligned}
$$


where $R\left[\psi_{p-2 k}\left(\overrightarrow{\operatorname{Tr}}^{k} f_{p}\right)\right]$ is the lifting of the $(p-2 k)$-form $\psi_{p-2 k}$ associated with the function $\overrightarrow{\operatorname{Tr}}^{k} f_{p}$ in $L_{s}^{2}\left(\mathbb{R}_{+}^{p-2 k}\right)$ and where $\left(\phi_{i}\right)$ is a CONS for $L^{2}\left(\mathbb{R}_{+}\right)$, $f_{p}=\sum_{i_{1}, \ldots, i_{p}=1}^{\infty} a_{i_{1}, \ldots, i_{p}} \phi_{i_{1}} \otimes \cdots \otimes \phi_{i_{p}}, \xi_{i}=I_{1}\left(\phi_{i}\right)$, and the sum over the $i$ 's in the third expression in (6.1) is interpreted on each $\Omega_{\sigma}$ as the limit in the space $L^{2}\left(\Omega_{\sigma}, P_{\sigma}\right)$ of the sequence

$$
\sum_{i_{2 k+1}, \ldots, i_{p}=1}^{N}\left(\sum_{j_{1}, \ldots, j_{k}=1}^{\infty} a_{j_{1}, j_{1}, \ldots, j_{k}, j_{k}, i_{2 k+1}, \ldots, i_{p}}\right) \xi_{i_{2 k+1}} \cdots \xi_{i_{p}} .
$$

Proof. We begin by establishing the first equality in (6.1). Our assumptions assure us, by Theorem 5.1, that the second expression in (6.1) makes sense and that formula $(5.1)$ can be applied to each function $R\left[\psi_{p-2 k}\left(\overrightarrow{\operatorname{Tr}}^{k} f_{p}\right)\right]$ in the second expression in (6.1). Our first equality below is obtained by doing this and using the consistency of the first and second order traces. The second equality comes simply from splitting off the $k=0, \nu=0$ term and the third equality from doing the remaining sum in a different order. Finally, the fourth equality comes from the Binomial Theorem;

$$
0=[1+(-1)]^{r}=r ! \sum_{k+\nu=r} \frac{(-1)^{k}}{k ! \nu !}
$$

We now carry out the steps which were just commented on above:

$$
\begin{aligned}
& \sum_{k=0}^{[p / 2]}(-1)^{k} \sigma^{2 k} C_{p, k} R\left[\psi_{p-2 k}\left(\overrightarrow{\operatorname{Tr}}^{k} f_{p}\right)\right] \\
& \quad=\sum_{k=0}^{[p / 2]} \frac{(-1)^{k} \sigma^{2 k} p !}{(p-2 k) ! 2^{k} k !} \sum_{\nu=0}^{\left[\frac{p-2 k}{2}\right]} \frac{(p-2 k) ! \sigma^{2 \nu}}{(p-2 k-2 \nu) ! 2^{\nu} \nu !} I_{p-2 k-2 \nu}^{\sigma}\left(\overrightarrow{\operatorname{Tr}}^{k+\nu} f_{p}\right) \\
& =I_{p}^{\sigma}\left(f_{p}\right)+\sum_{k=0}^{[p / 2]\left[\frac{p-2 k}{2}\right]} \sum_{\nu=0}^{(k, \nu) \neq(0,0)} \frac{(-1)^{k} p ! \sigma^{2(k+\nu)}}{(p-2 k-2 \nu) ! 2^{k+\nu} k ! \nu !} I_{p-2 k-2 \nu}^{\sigma}\left(\overrightarrow{\operatorname{Tr}}^{k+\nu} f_{p}\right) \\
& =I_{p}^{\sigma}\left(f_{p}\right)+\sum_{r=1}^{[p / 2]} \frac{p ! \sigma^{2 r}}{(p-2 r) ! 2^{r}}\left\{\sum_{k+\nu=r} \frac{(-1)^{k}}{k ! \nu !}\right\} I_{p-2 r}^{\sigma}\left(\overrightarrow{\operatorname{Tr}}^{r} f_{p}\right) \\
& =I_{p}^{\sigma}\left(f_{p}\right)
\end{aligned}
$$

as claimed.

It remains to establish the second equality in (6.1). By Proposition 3.3,

$$
\begin{aligned}
\overrightarrow{\operatorname{Tr}}^{k} f_{p}= & \sum_{i_{2 k+1}, \ldots, i_{p}=1}^{\infty} \\
& \cdot\left(\sum_{j_{1}, \ldots, j_{k}=1}^{\infty} a_{j_{1}, j_{1}, \ldots, j_{k}, j_{k}, i_{2 k+1}, \ldots, i_{p}}\right) \phi_{i_{2 k+1}} \otimes \cdots \otimes \phi_{i_{p}}
\end{aligned}
$$


so that

$$
\begin{aligned}
\psi_{p-2 k}\left(\overrightarrow{\operatorname{Tr}}^{k} f_{p}\right)(h)= & \sum_{i_{2 k+1}, \ldots, i_{p}=1}^{\infty} \\
& \cdot\left(\sum_{j_{1}, \ldots, j_{k}=1}^{\infty} a_{j_{1}, j_{1}, \ldots, j_{k}, j_{k}, i_{2 k+1}, \ldots, i_{p}}\right) \\
& \cdot\left(\phi_{i_{2 k+1}}, h\right) \cdots\left(\phi_{i_{p}}, h\right)
\end{aligned}
$$

and so by Theorem 5.1,

$$
\begin{aligned}
R\left[\psi_{p-2 k}\left(\overrightarrow{\operatorname{Tr}}^{k} f_{p}\right)\right]= & \sum_{i_{2 k+1}, \ldots, i_{p}=1}^{\infty} \\
& \cdot\left(\sum_{j_{1}, \ldots, j_{k}=1}^{\infty} a_{j_{1}, j_{1}, \ldots, j_{k}, j_{k}, i_{2 k+1}, \ldots, i_{p}}\right) \xi_{i_{2 k+1}} \cdots \xi_{i_{p}} .
\end{aligned}
$$

7. The NATURAL EXTENSION OF A WiENER-ITô INTEGRAL

The case $\sigma=1$ of Theorem 6.1 and the idea that the natural extension should preserve polynomials in the $\xi_{i}$ 's immediately suggests how to define $N\left[I_{p}\left(f_{p}\right)\right]$.

Definition 7.1. The natural extension of $I_{p}\left(f_{p}\right)$ is defined by

$$
\begin{aligned}
& N\left[I_{p}\left(f_{p}\right)\right]=\sum_{k=0}^{[p / 2]}(-1)^{k} C_{p, k} R\left[\psi_{p-2 k}\left(\overrightarrow{\operatorname{Tr}}^{k} f_{p}\right)\right]=\sum_{k=0}^{[p / 2]}(-1)^{k} C_{p, k} \\
& \cdot \sum_{i_{2 k+1}, \ldots, i_{p}=1}^{\infty}\left(\sum_{j_{1}, \ldots, j_{k}=1}^{\infty} a_{j_{1}, j_{1}, \ldots, j_{k}, j_{k}, i_{2 k+1}, \ldots, i_{p}}\right) \xi_{i_{2 k+1}} \ldots \xi_{i_{p}} .
\end{aligned}
$$

The next theorem gives us conditions under which Definition 7.1 makes sense. Theorem 7.1. Let $f_{p} \in L_{s}^{2}\left(\mathbb{R}_{+}^{p}\right)$ and let $\psi_{p}=\psi_{p}\left(f_{p}\right)$ be the associated p-form on $H=L^{2}\left(\mathbb{R}_{+}\right)$. Assume that $f_{p}$ has all its first and second order traces and that they are consistent.

Then the last two expressions in formula (7.1) are defined and agree s-a.s. and are scale-invariant measurable.

Proof. Apply Theorem 5.1 and Proposition 3.3 to each of the $(p-2 k)$-forms $\psi_{p-2 k}\left(\overrightarrow{\operatorname{Tr}}^{k} f_{p}\right), k=0,1, \ldots,[p / 2]$.

Next we give a theorem which will express the restriction of $N\left[I_{p}\left(f_{p}\right)\right]$ to $\Omega_{\sigma}$ as a sum of multiple Wiener-Itô integrals $I_{p-2 k}^{\sigma}\left(\overrightarrow{\operatorname{Tr}}^{k} f_{p}\right), k=0,1, \ldots,[p / 2]$. This theorem will be the basis of our calculation in the next section of the analytic Feynman integral of $N\left[I_{p}\left(f_{p}\right)\right]$.

Theorem 7.2. Let $f_{p} \in L_{s}^{2}\left(\mathbb{R}_{+}^{p}\right)$ and let $\psi_{p}=\psi_{p}\left(f_{p}\right)$ be the associated p-form on $H=L^{2}\left(\mathbb{R}_{+}\right)$. Assume that $f_{p}$ has all its first and second order traces and that they are consistent. 
Then, for any $\sigma>0$, we have $P_{\sigma}$-a.s. on $\Omega_{\sigma}$,

$$
N\left[I_{p}\left(f_{p}\right)\right]=\sum_{k=0}^{[p / 2]}\left(\sigma^{2}-1\right)^{k} C_{p, k} I_{p-2 k}^{\sigma}\left(\overrightarrow{\operatorname{Tr}}^{k} f_{p}\right) .
$$

Proof. Proposition 3.4 allows us to apply Theorem 6.1 to the right-hand side of (7.2); this yields the first equality below. The third equality comes from reordering the sum and the fourth from applying the Binomial Theorem to $(-1)^{r}=\left[\left(\sigma^{2}-1\right)+\left(-\sigma^{2}\right)\right]^{r}$. Finally the last equality comes from Definition 7.1 and Theorem 7.1 .

$$
\begin{aligned}
\sum_{k=0}^{[p / 2]}( & \left.\sigma^{2}-1\right)^{k} C_{p, k} I_{p-2 k}^{\sigma}\left(\overrightarrow{\operatorname{Tr}}^{k} f_{p}\right) \\
& =\sum_{k=0}^{[p / 2]} \frac{p !\left(\sigma^{2}-1\right)^{k}}{(p-2 k) ! 2^{k} k !}\left\{\sum_{\nu=0}^{\left[\frac{p-2 k}{2}\right]} \frac{(p-2 k) !(-1)^{\nu} \sigma^{2 \nu}}{(p-2 k-2 \nu) ! 2^{\nu} \nu !} R\left[\psi_{p-2 k-2 \nu}\left(\overrightarrow{\operatorname{Tr}}^{k+\nu} f_{p}\right)\right]\right\} \\
& =\sum_{k=0}^{[p / 2]\left[\frac{p-2 k}{2}\right]} \frac{p !\left(\sigma^{2}-1\right)^{k}(-1)^{\nu} \sigma^{2 \nu}}{(p-2 k-2 \nu) ! 2^{k+\nu} k ! \nu !} R\left[\psi_{p-2 k-2 \nu}\left(\overrightarrow{\operatorname{Tr}}^{k+\nu} f_{p}\right)\right] \\
& =\sum_{r=0}^{[p / 2]} \frac{p !}{(p-2 r) ! 2^{r}}\left\{\sum_{k+\nu=r} \frac{\left(\sigma^{2}-1\right)^{k}\left(-\sigma^{2}\right)^{\nu}}{k ! \nu !}\right\} R\left[\psi_{p-2 r}\left(\overrightarrow{\operatorname{Tr}}^{r} f_{p}\right)\right] \\
& =\sum_{r=0}^{[p / 2]}(-1)^{r} C_{p, r} R\left[\psi_{p-2 r}\left(\overrightarrow{\operatorname{Tr}}^{r} f_{p}\right)\right] \\
& =N\left[I_{p}\left(f_{p}\right)\right]
\end{aligned}
$$

as desired.

Remark 7.1. Formula (7.2) is closely related to (8) in [5], a key formula in the paper of $\mathrm{Hu}$ and Meyer. However, in [5], the right-hand side of (7.2) is their "suggested definition" of the natural extension of $I_{p}\left(f_{p}\right)$ to $\Omega_{\sigma}$. Hu and Meyer do not have one formula (like our (7.1)) which extends $I_{p}\left(f_{p}\right)$ to $\Omega_{\sigma}$ for all $\sigma>0$. Their "suggested definition" has become a theorem in our development. Further, our Definition 7.1 reflects in a rather transparent way the desire to extend $I_{p}\left(f_{p}\right)$ so as to preserve polynomials in the $\xi_{i}$ 's.

Next we give a simple example which will illustrate our formulas and help to clarify the idea of the natural extension and the concept of functions equal $s$-a.s. Let $\phi:=\chi_{[0,1]}$ so that $f_{1}:=\phi \otimes \phi$ belongs to $L_{s}^{2}\left(\mathbb{R}_{+}^{2}\right)$. We introduce a function $G: \mathscr{C}_{0}\left(\mathbb{R}_{+}\right) \rightarrow \mathbb{R}$ which is everywhere defined and continuous and with which we will compare two different extensions of $I_{2}\left(f_{2}\right): \Omega_{1} \rightarrow \mathbb{R}$. Let $G(x):=x^{2}(1)-1$.

Using the third expression in (6.1) (or (4.7), the corresponding formula for finite expansions) with $\sigma=1, p=2, k=0,1$, we see that $P_{1}$-a.s. on $\Omega_{1}$,

$$
\begin{aligned}
I_{2}\left(f_{2}\right)(x) & =\xi^{2}(x)-1=\left[\int_{\mathbb{R}_{+}} \phi(x) d x(s)\right]^{2}-1 \\
& =\left[\int_{0}^{1} d x(s)\right]^{2}-1=x^{2}(1)-1
\end{aligned}
$$

where $\xi=I_{1}(\phi)$. Hence $I_{2}\left(f_{2}\right)=G \quad P_{1}$-a.s. on $\Omega_{1}$. 
By (7.1), $N\left[I_{2}\left(f_{2}\right)\right]=\xi^{2}-1$. In particular, for every $\sigma>0, P_{1}$-a.s. on $\Omega_{1}$,

$$
N\left[I_{2}\left(f_{2}\right)\right](\sigma x)=\xi^{2}(\sigma x)-1=\sigma^{2} \xi^{2}(x)-1=\sigma^{2} x^{2}(1)-1 .
$$

We can also obtain the formula just above by using (7.2) of Theorem 7.2: For every $\sigma>0, P_{1}$-a.s. on $\Omega_{1}$,

$$
\begin{aligned}
N\left[I_{2}\left(f_{2}\right)\right](\sigma x) & =C_{2,0} I_{2}^{\sigma}\left(\overrightarrow{\operatorname{Tr}}^{0} f_{2}\right)(\sigma x)+\left(\sigma^{2}-1\right) C_{2,1} I_{0}^{\sigma}\left(\overrightarrow{\operatorname{Tr}}^{1} f_{2}\right)(x) \\
& =I_{2}^{\sigma}\left(f_{2}\right)(\sigma x)+\left(\sigma^{2}-1\right) \overrightarrow{\operatorname{Tr}}^{1} f_{2}=\sigma^{2} I_{2}\left(f_{2}\right)(x)+\left(\sigma^{2}-1\right) \\
& =\sigma^{2}\left[\xi^{2}(x)-1\right]+\sigma^{2}-1=\sigma^{2} \xi^{2}(x)-1=\sigma^{2} x^{2}(1)-1 .
\end{aligned}
$$

Note that $N\left[I_{2}\left(f_{2}\right)\right]=G$ s-a.s.

In contrast, we define the extension $F$ of $I_{2}\left(f_{2}\right)$ to $\bigcup_{\sigma>0} \Omega_{\sigma}$ by $F(\sigma x)=$ $I_{2}^{\sigma}\left(f_{2}\right)(\sigma x)$. Now $P_{1}$-a.s. on $\Omega_{1}, F(\sigma x)=\sigma^{2} I_{2}\left(f_{2}\right)(x)=\sigma^{2}\left[\xi^{2}(x)-1\right]=$ $\sigma^{2} x^{2}(1)-\sigma^{2}$.

Summarizing the above: $N\left[I_{2}\left(f_{2}\right)\right]=G \quad s$-a.s. and $N\left[I_{2}\left(f_{2}\right)\right]=G=F \quad P_{1}$ a.s. However, for every $\sigma \neq 1$, it is not true that $F=G P_{\sigma}$-a.s., and, in fact,

$$
P_{\sigma}\left\{y \in \Omega_{\sigma}: F(y) \neq G(y)\right\}=P_{1}\left\{x \in \Omega_{1}: F(\sigma x) \neq G(\sigma x)\right\}=1
$$

since $G(\sigma x)=\sigma^{2} x^{2}(1)-1$ for every $x \in \Omega_{1}$ whereas $F(\sigma x)=\sigma^{2} x^{2}(1)-\sigma^{2}$ $P_{1}$-a.s. on $\Omega_{1}$.

We finish this section by discussing briefly a generalization of the results above. This generalization is related to a further formula of $\mathrm{Hu}$ and Meyer [5]. Let $f_{p} \in L_{s}^{2}\left(\mathbb{R}_{+}^{p}\right)$. We will define the natural extension $N\left[I_{p}^{\tau}\left(f_{p}\right)\right]$ for any $\tau>0$. Again, Theorem 6.1 suggests how this should be done.

Definition 7.2. The natural extension of $I_{p}^{\tau}\left(f_{p}\right)$ is defined by

$$
\begin{aligned}
N\left[I_{p}^{\tau}\left(f_{p}\right)\right]= & \sum_{k=0}^{[p / 2]}(-1)^{k} \tau^{2 k} C_{p, k} R\left[\psi_{p-2 k}\left(\overrightarrow{\operatorname{Tr}}^{k} f_{p}\right)\right] \\
= & \sum_{k=0}^{[p / 2]}(-1)^{k} \tau^{2 k} C_{p, k} \sum_{i_{2 k+1}, \ldots, i_{p}=1}^{\infty} \\
& \cdot\left(\sum_{j_{1}, \ldots, j_{k}=1}^{\infty} a_{j_{1}, j_{1}, \ldots, j_{k}, j_{k}, i_{2 k+1}, \ldots, i_{p}}\right) \xi_{i_{2 k+1}} \ldots \xi_{i_{p}} .
\end{aligned}
$$

Under the assumption that $f_{p}$ has all its first and second order traces and they are consistent, we can show just as in Theorem 7.1 that Definition 7.2 makes sense. Next we ask for a formula extending (7.2).

Theorem 7.3. Under the assumption of Theorem 7.2, for any $\sigma>0$, we have $P_{\sigma}$-a.s. on $\Omega_{\sigma}$,

$$
N\left[I_{p}^{\tau}\left(f_{p}\right)\right]=\sum_{k=0}^{[p / 2]}\left(\sigma^{2}-\tau^{2}\right)^{k} C_{p, k} I_{p-2 k}^{\sigma}\left(\overrightarrow{\operatorname{Tr}}^{k} f_{p}\right) .
$$

Proof. The argument proceeds just as in the proof of Theorem 7.2 except that here one applies the Binomial Theorem to

$$
\left[-\tau^{2}\right]^{r}=\left[\left(\sigma^{2}-\tau^{2}\right)+\left(-\sigma^{2}\right)\right]^{r}
$$




\section{The Feynman integral of the NatUral eXtension OF A WIENER-ITÔ INTEGRAL}

We are now able to solve the problem which we discussed at the beginning of $\S 1$. The reader may wish to review that material.

Theorem 8.1. Let $f_{p} \in L_{s}^{2}\left(\mathbb{R}_{+}^{p}\right)$ and let $\psi_{p}=\psi_{p}\left(f_{p}\right)$ be the associated p-form on $H=L^{2}\left(\mathbb{R}_{+}\right)$. Assume that $f_{p}$ has all its first and second order traces and that they are consistent.

Then, for every $\sigma>0$, we have the formula

$$
\int_{\mathscr{C}_{0}\left(\mathbb{R}_{+}\right)} \frac{1}{p !} N\left[I_{p}\left(f_{p}\right)\right](\sigma x) d P_{1}(x)=\left\{\begin{array}{l}
\frac{\left(\sigma^{2}-1\right)^{k}}{2^{k} k !} \overrightarrow{\operatorname{Tr}}^{k}\left(f_{2 k}\right) \text { if } p=2 k \text { is even, } \\
0 \text { if } p \text { is odd. }
\end{array}\right.
$$

Further, the analytic Feynman integral of the function $N\left[I_{p}\left(f_{p}\right)\right] / p$ ! is given by the right-hand side of (8.1) with $\sigma^{2}=-i$.

Proof. Formula (7.2) of Theorem 7.2 is the key to the proof. Fix $\sigma>0$. The steps below follow from a change of variables, an application of (7.2) and the fact that, for $p \geq 1$,

$$
\begin{aligned}
\int_{\mathscr{C}_{0}\left(\mathbf{R}_{+}\right)} I_{p}^{\sigma}\left(f_{p}\right)(x) d P_{\sigma}(x) & =0: \int_{\mathscr{C}_{0}\left(\mathbf{R}_{+}\right)} \frac{1}{p !} N\left[I_{p}\left(f_{p}\right)\right](\sigma x) d P_{1}(x) \\
& =\int_{\mathscr{C}_{0}\left(\mathbf{R}_{+}\right)} \frac{1}{p !} N\left[I_{p}\left(f_{p}\right)\right](x) d P_{\sigma}(x) \\
& =\frac{1}{p !} \sum_{\nu=0}^{[p / 2]} \frac{\left(\sigma^{2}-1\right)^{\nu} p !}{(p-2 \nu) ! 2^{\nu} \nu !} \int_{\mathscr{E}_{0}\left(\mathbf{R}_{+}\right)} I_{p-2 \nu}^{\sigma}\left(\overrightarrow{\operatorname{Tr}}^{\nu} f_{p}\right)(x) d P_{\sigma}(x) \\
& =\left\{\begin{array}{l}
\frac{\left(\sigma^{2}-1\right)^{k}}{2^{k} k !} \overrightarrow{\operatorname{Tr}}^{k} f_{2 k} \text { if } p=2 k \text { is even, } \\
0 \text { if } p \text { is odd. }
\end{array}\right.
\end{aligned}
$$

which is the desired formula.

Remark 8.1. It is natural to investigate the class of functions $f$ which possess a Feynman integral in the sense of [5] and, in particular, to ask if various functions of interest in quantum mechanics fall in this class. Such matters are discussed to some extent in [5] and are of interest to the authors but they have not been our concern in this paper.

We finish this section by stating a result like Theorem 8.1 except that it involves the natural extension of $I_{p}^{\tau}\left(f_{p}\right)$ rather than $N\left[I_{p}\left(f_{p}\right)\right]$.

Theorem 8.2. Let the assumptions of Theorem 8.1 be satisfied and let $\tau>0$ be given. Then, for every $\sigma>0$, we have the formula

$$
\int_{\mathscr{C}_{0}\left(\mathbb{R}_{+}\right)} \frac{1}{p !} N\left[I_{p}^{\tau}\left(f_{p}\right)\right](\sigma x) d P_{1}(x)=\left\{\begin{array}{c}
\frac{\left(\sigma^{2}-\tau^{2}\right)^{k}}{2^{k} k !} \overrightarrow{\operatorname{Tr}}^{k}\left(f_{2 k}\right) \\
\quad \text { if } p=2 k \text { is even, } \\
0 \quad \text { if } p \text { is odd. }
\end{array}\right.
$$

Proof. The proof is the same as the proof of Theorem 8.1 except that formula (7.5) is used instead of (7.2). 
Remark 8.2. If we analytically continue the right-hand side of $(8.2)$ to $\sigma^{2}=-i$, we obtain the analytic Feynman integral of $N\left[I_{p}^{\tau}\left(f_{p}\right)\right] / p !$.

\section{FURTHER RESUltS ON $k$-TRACES}

We concentrated our attention in $\S 3$ on the limiting $k$-trace, $\overrightarrow{\operatorname{Tr}}^{k} f_{p}$, since our principal results on the lifting of $p$-forms, the existence of the natural extension of $I_{p}\left(f_{p}\right)$, etc. all heavily involve $\overrightarrow{\operatorname{Tr}}^{k} f_{p}$ where $f_{p} \in L_{s}^{2}\left(\mathbb{R}_{+}^{p}\right)$. A further $k$-trace, $\operatorname{Tr}^{k} f_{p}$, was defined (see Definition 3.1) and played an important but subsidiary role. In this section we will give two more definitions of $k$-trace, the iterated $k$-trace, $\operatorname{Tr}_{i}^{k} f_{p}$ and the tensorial $k$-trace, $\operatorname{Tr}_{t}^{k} f_{p}$. It is the iterated $k$-trace which appears in the paper of $\mathrm{Hu}$ and Meyer [5]. The tensorial $k$-trace also seems to be a natural object of study. In particular, it will help us to understand some of the relationships between the other three $k$-traces. It is $\operatorname{Tr}^{k} f_{p}$ which is associated in the most straightforward way with the familiar concept of a trace class operator. This is discussed in some detail in [8, §2] and in [17] and we will not go into it here. Most of the results of this section, in addition to being of interest in their own right, either lead to or provide sufficient conditions under which the hypotheses of earlier theorems are satisfied. Indeed, the limiting $k$-trace is explicitly involved starting with Theorem 9.2.

Before beginning formally, we mention a few of the results of this section. It is an immediate consequence of the definitions, as we will see below, that the existence of $\operatorname{Tr}^{k} f_{p}$ implies the existence of $\operatorname{Tr}_{t}^{k} f_{p}$ and equality. Among other results of this section, we will show that the existence of $\operatorname{Tr}_{t}^{k} f_{p}$ implies the existence of $\overrightarrow{\operatorname{Tr}}^{k} f_{p}$ and equality. Further, we show that if all of $\operatorname{Tr}_{t}^{1} f_{p}, \ldots, \operatorname{Tr}_{t}^{\nu} f_{p}$ exist, then all of $\operatorname{Tr}_{i}^{1} f_{p}, \ldots, \operatorname{Tr}_{i}^{\nu} f_{p}$ exist and equality holds. Finally, we show that if there exists a CONS $\left(\phi_{i}\right)$ for $L^{2}\left(\mathbb{R}_{+}\right)$such that the coeffcients in the expansion of $f_{p}$ in terms of the CONS $\left(\phi_{i_{1}} \otimes \cdots \otimes \phi_{i_{p}}\right)$ for $L^{2}\left(\mathbb{R}_{+}^{p}\right)$ are in $l_{1}$, then all four $k$-traces exist and are equal.

We begin with the definition of the tensorial $k$-trace.

Definition 9.1. Let $f_{p} \in L_{s}^{2}\left(\mathbb{R}_{+}^{p}\right)$. We take $\operatorname{Tr}_{t}^{0} f_{p}=f_{p}$ and, for $1 \leq k \leq[p / 2]$, we say that $\operatorname{Tr}_{t}^{k} f_{p}$ exists and equals $h \in L_{s}^{2}\left(\mathbb{R}_{+}^{p-2 k}\right)$ if and only if for any $k$ CONS $\left(\phi_{i_{1}}^{(1)}\right), \ldots,\left(\phi_{i_{k}}^{(k)}\right)$ for $L^{2}\left(\mathbb{R}_{+}\right)$and for any enumeration $e_{1}, e_{2}, \ldots$ of the tensorial CONS $\left(\phi_{i_{1}}^{(1)} \otimes \cdots \otimes \phi_{i_{k}}^{(k)}\right)$ for $L^{2}\left(\mathbb{R}_{+}^{k}\right)$,

$$
\begin{gathered}
\sum_{j=1}^{\infty} \int_{\mathbf{R}_{+}^{k} \times \mathbf{R}_{+}^{k}} f_{p}\left(s_{1}, \ldots, s_{k}, s_{k+1}, \ldots, s_{2 k}, \cdots, \cdot\right) e_{j}\left(s_{j}, \ldots, s_{k}\right) \\
\quad e_{j}\left(s_{k+1}, \ldots, s_{2 k}\right) d s_{1} \cdots d s_{k} d s_{k+1} \cdots d s_{2 k}=h(\cdot),
\end{gathered}
$$

where the series on the left-hand side of $(9.1)$ converges to $h$ in the norm of $L^{2}\left(\mathbb{R}_{+}^{p-2 k}\right)$.

The first proposition follows immediately from Definitions 3.1 and 9.1. We state it formally for ease of reference.

Proposition 9.1. The existence of $\operatorname{Tr}^{k} f_{p}$ implies the existence of $\operatorname{Tr}_{t}^{k} f_{p}$ and equality. Also $\operatorname{Tr}^{1} f_{p}$ exists if and only if $\operatorname{Tr}_{t}^{1} f_{p}$ exists.

Our next proposition assures us that when $\operatorname{Tr}_{t}^{k} f_{p}$ exists, it can be expressed either as a multiple series or as an iterated series. This is not especially difficult 
to prove using known results, but, since much of the rest of this section depends on it, we will give some details of the proof.

Proposition 9.2. Let $f_{p} \in L_{s}^{2}\left(\mathbb{R}_{+}^{p}\right)$ and let $1 \leq k \leq[p / 2]$. If $\operatorname{Tr}_{t}^{k} f_{p}$ exists and if $\left(\phi_{i}^{(1)}\right), \ldots,\left(\phi_{i}^{(k)}\right)$ are any $k$ CONS for $L^{2}\left(\mathbb{R}_{+}\right)$, then $\operatorname{Tr}_{t}^{k} f_{p}$ can be expressed as the multiple series,

$$
\begin{array}{r}
\operatorname{Tr}_{t}^{k} f_{p}=\sum_{i_{1}, \ldots, i_{k}=1}^{\infty} \int_{\mathbf{R}_{+}^{k} \times \mathbf{R}_{+}^{k}} f_{p}\left(s_{1}, \ldots, s_{k}, s_{k+1}, \ldots, s_{2 k}, \cdot, \ldots, \cdot\right) \phi_{i_{1}}^{(1)}\left(s_{1}\right) \phi_{i_{1}}^{(1)}\left(s_{2}\right) \\
\cdots \cdots \phi_{i_{k}}^{(k)}\left(s_{2 k-1}\right) \phi_{i_{k}}^{(k)}\left(s_{2 k}\right) d s_{1} \cdots d s_{k} d s_{k+1} \cdots d s_{2 k},
\end{array}
$$

or as an iterated series where the iteration can be done in whatever order is desired; for example

$$
\begin{array}{r}
\operatorname{Tr}_{t}^{k} f_{p}=\sum_{i_{k}=1}^{\infty} \cdots \sum_{i_{1}=1}^{\infty} \int_{\mathbf{R}_{+}^{k} \times \mathbf{R}_{+}^{k}} f_{p}\left(s_{1}, \ldots, s_{k}, s_{k+1}, \ldots, s_{2 k}, \cdot, \ldots, \cdot\right) \phi_{i_{1}}^{(1)}\left(s_{1}\right) \phi_{i_{1}}^{(1)}\left(s_{2}\right) \\
\cdots \cdots \phi_{i_{k}}^{(k)}\left(s_{2 k-1}\right) \phi_{i_{k}}^{(k)}\left(s_{2 k}\right) d s_{1} \cdots d s_{k} d s_{k+1} \cdots d s_{2 k} .
\end{array}
$$

The series in (9.2) and (9.3) converge to $\operatorname{Tr}_{t}^{k} f_{p}$ in the sense of the norm on $L^{2}\left(\mathbb{R}_{+}^{p-2 k}\right)$.

Proof. Take any tensorial CONS as described in Definition 9.1 and enumerate it; $\operatorname{Tr}_{t}^{k} f_{p}$ is given by (9.1). Since any permutation of a CONS is again a CONS, we see that the series (9.1) converges unconditionally. By Proposition 1.C.1, p. 15 in [16], for every $\varepsilon>0$ there exists an integer $N$ so that for every finite set of integers $F$ which satisfies $\min \{j \in F\}>N$ we have

$$
\left\|\sum_{j \in F} X_{j}\right\|_{L^{2}\left(\mathbb{R}_{+}^{p-2 k}\right)}<\varepsilon,
$$

where $X_{j}$ is the $j$ th term of the series (9.1). It is not hard to see that this is equivalent to the Cauchy condition for what is sometimes called "unordered summation": For every $\varepsilon>0$ there exists a finite subset $F_{1}$ of the (in this case countable) index set such that for every finite subset $F$ of the index set which is disjoint from $F_{1}$ we have

$$
\left\|\sum_{j \in F} X_{j}\right\|_{L_{s}^{2}\left(\mathbb{R}_{+}^{p-2 k}\right)}<\varepsilon .
$$

This tells us that any ordering which one may have on the index set is essentially irrelevant and the limit is the limit of a net where the associated directed set $\mathscr{D}$ is the collection of all finite subsets of the index set where $F_{2}$ follows $F_{1}$ if and only if $F_{2} \supseteq F_{1}$. Now the collection of all sets of the form

$$
\left\{1, \ldots, N_{1}\right\} \times\left\{1, \ldots, N_{2}\right\} \times \cdots \times\left\{1, \ldots, N_{k}\right\}
$$

is a cofinal subset $\left[15\right.$, p. 66] of the directed set $\mathscr{D}$ and so $\operatorname{Tr}_{t}^{k} f_{p}$ is the limit as $N_{1} \rightarrow \infty, \ldots, N_{k} \rightarrow \infty$ of the sums

$$
\begin{aligned}
& \sum_{i_{1}, \ldots, i_{k}=1}^{N_{1}, \ldots, N_{k}} \int_{\mathbb{R}_{+}^{k} \times \mathbb{R}_{+}^{k}} f_{p}\left(s_{1}, \ldots, s_{k}, s_{k+1}, \ldots, s_{2 k}, \cdot, \ldots, \cdot\right) \\
& \quad \cdot \phi_{i_{1}}^{(1)}\left(s_{1}\right) \phi_{i_{1}}^{(1)}\left(s_{2}\right) \cdots \phi_{i_{k}}^{(k)}\left(s_{2 k-1}\right) \phi_{i_{k}}^{(k)}\left(s_{2 k}\right) d s_{1} \cdots d s_{k} d s_{k+1} \cdots d s_{2 k}
\end{aligned}
$$

that is, (9.2) holds. 
Now part (a) of $[15,6 . S$, p. 214] tells us that a function which has an unordered sum over a certain index set will also have an unordered sum over any subset of that index set. Using this and (c) of [15, 6.S, p. 214] which deals with iterated sums we see that $\operatorname{Tr}_{t}^{k} f_{p}$ can be written as an iterated sum in any order and, in particular, that (9.3) holds.

Corollary 9.1. If $\operatorname{Tr}^{k} f_{p}$ exists and $\left(\phi_{i}^{(1)}\right), \ldots,\left(\phi_{i}^{(k)}\right)$ are CONSs for $L^{2}\left(\mathbb{R}_{+}\right)$as in Proposition 9.2, then $\operatorname{Tr}^{k} f_{p}$ is given by both of (9.2) and (9.3).

Proof. This follows immediately from Propositions 9.1 and 9.2.

Next we give the definition of the iterated $k$-trace, $\operatorname{Tr}_{i}^{k} f_{p}$.

Definition 9.2. Let $f_{p} \in L_{s}^{2}\left(\mathbb{R}_{+}^{p}\right)$. We take $\operatorname{Tr}_{i}^{0} f_{p}=f_{p}$ and we let $\operatorname{Tr}_{i}^{1} f_{p}:=$ $\operatorname{Tr}^{1} f_{p}$ provided that $\operatorname{Tr}^{1} f_{p}$ exists. When $\operatorname{Tr}^{1} f_{p}$ exists, it belongs to $L_{s}^{2}\left(\mathbb{R}_{+}^{p-2}\right)$ and we may attempt to take its 1-trace, $\operatorname{Tr}^{1}\left(\operatorname{Tr}^{1} f_{p}\right)$. If $\operatorname{Tr}^{1}\left(\operatorname{Tr}^{1} f_{p}\right)$ exists, we let $\operatorname{Tr}_{i}^{2} f_{p}:=\operatorname{Tr}^{1}\left(\operatorname{Tr}^{1} f_{p}\right)$. This procedure is continued inductively and for $1 \leq k \leq[p / 2]$,

$$
\operatorname{Tr}_{i}^{k} f_{p}:=\operatorname{Tr}^{1}\left(\operatorname{Tr}^{1}\left(\cdots\left(\operatorname{Tr}^{1}\left(\operatorname{Tr}^{1} f_{p}\right)\right) \cdots\right)\right)
$$

provided that all $k$ 1-traces involved exist.

The next proposition follows immediately from the iterative nature of $\operatorname{Tr}_{i}^{k} f_{p}$.

Proposition 9.3. Let $f_{p} \in L_{s}^{2}\left(\mathbb{R}_{+}^{p}\right)$ and suppose that $\operatorname{Tr}_{i}^{r} f_{p}$ exists where $1 \leq r \leq$ [p/2]. Then

(i) $\operatorname{Tr}_{i}^{k} f_{p}$ exists for all $1 \leq k \leq r$.

(ii) If $0 \leq k+\nu \leq r$, then $\operatorname{Tr}_{i}^{k} f_{p}, \operatorname{Tr}_{i}^{\nu}\left(\operatorname{Tr}_{i}^{k} f_{p}\right)$ and $\operatorname{Tr}_{i}^{k+\nu} f_{p}$ all exist and

$$
\operatorname{Tr}_{i}^{k+\nu} f_{p}=\operatorname{Tr}_{i}^{\nu}\left(\operatorname{Tr}_{i}^{k} f_{p}\right)
$$

We do not know if the existence of the tensorial $k$-trace implies the existence of the iterated $k$-trace, but our next result assures us that if $\operatorname{Tr}_{t}^{1} f_{p}, \ldots, \operatorname{Tr}_{t}^{\nu} f_{p}$ all exist, then $\operatorname{Tr}_{i}^{1} f_{p}, \ldots, \operatorname{Tr}_{i}^{\nu} f_{p}$ all exist.

Theorem 9.1. Let $f_{p} \in L_{s}^{2}\left(\mathbb{R}_{+}^{p}\right)$ and suppose that $\operatorname{Tr}_{t}^{1} f_{p}, \ldots, \operatorname{Tr}_{t}^{\nu} f_{p}$ all exist where $1 \leq \nu \leq[p / 2]$. Then $\operatorname{Tr}_{i}^{1} f_{p}, \ldots, \operatorname{Tr}_{i}^{\nu} f_{p}$ all exist and

$$
\operatorname{Tr}_{i}^{k} f_{p}=\operatorname{Tr}_{t}^{k} f_{p}, \quad k=1, \ldots, \nu .
$$

Proof. $\operatorname{Tr}_{i}^{1} f_{p}$ is exactly the same object as $\operatorname{Tr}_{t}^{1} f_{p}$ and so there is nothing to prove if $\nu=1$. Suppose that $\operatorname{Tr}_{t}^{1} f_{p}$ and $\operatorname{Tr}_{t}^{2} f_{p}$ exist. Then $\operatorname{Tr}_{i}^{1} f_{p}$ exists and we must show that $\operatorname{Tr}_{i}^{2} f_{p}$ exists and equals $\operatorname{Tr}_{t}^{2} f_{p}$. Let any two CONs $\left(\phi_{i}^{(1)}\right)$ and $\left(\phi_{i}^{(2)}\right)$ for $L^{2}\left(\mathbb{R}_{+}\right)$be given. Also let $\varepsilon>0$ be given. Since $\operatorname{Tr}_{t}^{2} f_{p}$ exists there are, by Proposition 9.2, integers $N_{1}$ and $N_{2}$ such that if $n_{1} \geq N_{1}$ and $n_{2} \geq N_{2}$ then

$$
\begin{aligned}
& \| \sum_{i_{1}, i_{2}=1}^{n_{1}, n_{2}} \int_{\mathbb{R}_{+}^{2} \times \mathbb{R}_{+}^{2}} f_{p}\left(s_{1}, \ldots, s_{4}, \cdot, \ldots, \cdot\right) \\
& \qquad \phi_{i_{1}}^{(1)}\left(s_{1}\right) \phi_{i_{1}}^{(1)}\left(s_{2}\right) \phi_{i_{2}}^{(2)}\left(s_{3}\right) \phi_{i_{2}}^{(2)}\left(s_{4}\right) d s_{1} \cdots d s_{4}-\operatorname{Tr}_{t}^{2} f_{p} \|_{L^{2}\left(\mathbb{R}_{+}^{p-4}\right)}<\varepsilon .
\end{aligned}
$$


Alternately, we can write,

(9.8)

$$
\begin{gathered}
\| \sum_{i_{2}=1}^{n_{2}}\left\{\int_{\mathbb{R}_{+} \times \mathbf{R}_{+}}\left[\sum_{i_{1}=1}^{n_{1}} \int_{\mathbb{R}_{+} \times \mathbb{R}_{+}} f_{p}\left(s_{1}, \ldots, s_{4}, \cdot, \ldots, \cdot\right) \phi_{i_{1}}^{(1)}\left(s_{1}\right) \phi_{i_{1}}^{(1)}\left(s_{2}\right) d s_{1} d s_{2}\right]\right. \\
\left.\cdot \phi_{i_{2}}^{(2)}\left(s_{3}\right) \phi_{i_{2}}^{(2)}\left(s_{4}\right) d s_{3} d s_{4}\right\}-\operatorname{Tr}_{t}^{2} f_{p} \|_{L^{2}\left(\mathbf{R}_{+}^{p-4}\right)}^{<\varepsilon}<
\end{gathered}
$$

Fix $n_{2} \geq N_{2}$. Now since $\operatorname{Tr}_{i}^{1} f_{p}$ exists,

$$
\sum_{i_{1}=1}^{n_{1}} \int_{\mathbb{R}_{+} \times \mathbb{R}_{+}} f_{p}\left(s_{1}, s_{2}, \cdot, \ldots, \cdot\right) \phi_{i_{1}}^{(1)}\left(s_{1}\right) \phi_{i_{1}}^{(1)}\left(s_{2}\right) d s_{1} d s_{2} \rightarrow \operatorname{Tr}_{i}^{1} f_{p}
$$

in $L^{2}\left(\mathbb{R}_{+}^{p-2}\right)$ norm as $n_{1} \rightarrow \infty$. Hence the sequence of integral operators whose kernels are given by the left-hand side of (9.9) converges in HilbertSchmidt norm to the integral operator with kernel $\operatorname{Tr}_{i}^{1} f_{p}$. (We think of these operators as acting on $L^{2}\left(\mathbb{R}_{+}^{2}\right)$ and, in this case, as having range in $L^{2}\left(\mathbb{R}_{+}^{p-4}\right)$.) Thus these operators converge in operator norm and so in the strong operator topology. Therefore

$$
\begin{aligned}
& \sum_{i_{2}=1}^{n_{2}}\left\{\int _ { \mathbf { R } _ { + } \times \mathbf { R } _ { + } } \left[\sum_{i_{1}=1}^{n_{1}} \int_{\mathbf{R}_{+} \times \mathbf{R}_{+}} f_{p}\left(s_{1}, s_{2}, s_{3}, s_{4}, \cdot, \ldots, \cdot\right)\right.\right. \\
& \left.\left.\cdot \phi_{i_{1}}^{(1)}\left(s_{1}\right) \phi_{i_{1}}^{(1)}\left(s_{2}\right) d s_{1} d s_{2}\right] \phi_{i_{2}}^{(2)}\left(s_{3}\right) \phi_{i_{2}}^{(2)}\left(s_{4}\right) d s_{3} d s_{4}\right\} \\
& \rightarrow \sum_{i_{2}=1}^{n_{2}}\left\{\int_{\mathbf{R}_{+} \times \mathbf{R}_{+}}\left(\operatorname{Tr}_{i}^{1} f_{p}\right)\left(s_{3}, s_{4}, \cdot \ldots, \cdot,\right) \phi_{i_{2}}^{(2)}\left(s_{3}\right) \phi_{i_{2}}^{(2)}\left(s_{4}\right) d s_{3} d s_{4}\right\}
\end{aligned}
$$

in $L^{2}\left(\mathbb{R}_{+}^{p-4}\right)$ norm as $n_{1} \rightarrow \infty$. Now (9.10) allows us to take the limit as $n_{1} \rightarrow \infty$ in (9.8) and we obtain

$$
\| \sum_{i_{2}=1}^{n_{2}}\left\{\int_{\mathbb{R}_{+} \times \mathbb{R}_{+}}\left(\operatorname{Tr}_{i}^{1} f_{p}\right)\left(s_{3}, s_{4}, \cdot, \ldots, \cdot\right)\right.
$$

$$
\left.\cdot \phi_{i_{2}}^{(2)}\left(s_{3}\right) \phi_{i_{2}}^{(2)}\left(s_{4}\right) d s_{3} d s_{4}\right\}-\operatorname{Tr}_{t}^{2} f_{p} \|_{L^{2}\left(\mathbb{R}_{+}^{p-4}\right)} \leq \varepsilon .
$$

It follows that $\operatorname{Tr}_{i}^{2} f_{p}$ exists and equals $\operatorname{Tr}_{t}^{2} f_{p}$ as desired. We continue inductively like this to get the result.

Our next result is an immediate corollary of the preceding theorem and Proposition 9.1. Corollary 9.8 below will give additional information under the same assumptions.

Corollary 9.2. Let $f_{p} \in L_{s}^{2}\left(\mathbb{R}_{+}^{p}\right)$ and suppose that $\operatorname{Tr}^{1} f_{p}, \ldots, \operatorname{Tr}^{\nu} f_{p}$ all exist where $1 \leq \nu \leq[p / 2]$. Then $\operatorname{Tr}_{t}^{1} f_{p}, \ldots, \operatorname{Tr}_{t}^{\nu} f_{p}$ and $\operatorname{Tr}_{i}^{1} f_{p}, \ldots, \operatorname{Tr}_{i}^{\nu} f_{p}$ all exist and for $k=1, \ldots, \nu$ we have

$$
\operatorname{Tr}^{k} f_{p}=\operatorname{Tr}_{t}^{k} f_{p}=\operatorname{Tr}_{i}^{k} f_{p}
$$

Next we define "second order tensorial traces." The definition follows the pattern for second order limiting traces (see Definition 3.3). 
Definition 9.3. Let $f_{p} \in L_{s}^{2}\left(\mathbb{R}_{+}^{p}\right)$. If $\operatorname{Tr}_{t}^{k} f_{p}$ exists for $k=0,1, \ldots,[p / 2]$, we say that $f_{p}$ has all of its first order traces. Whenever $\operatorname{Tr}_{t}^{k} f_{p}$ exists, it belongs to $L_{s}^{2}\left(\mathbb{R}_{+}^{p-2 k}\right)$, and, for $\nu=0,1, \ldots,[(p-2 k) / 2]$, it may possess a $\nu$-trace, $\operatorname{Tr}_{t}^{\nu}\left[\operatorname{Tr}_{t}^{k} f_{p}\right]$. If all of these traces exist, $k=0,1, \ldots,[p / 2]$ and $\nu=0,1, \ldots$, $[(p-2 k) / 2]$, we say that $f_{p}$ has all of its second order tensorial traces. The second order traces are said to be consistent with the first order traces provided that $\operatorname{Tr}_{t}^{\nu}\left[\operatorname{Tr}_{t}^{k} f_{p}\right]=\operatorname{Tr}_{t}^{k+\nu} f_{p}$ for $k=0,1, \ldots,[p / 2]$ and $\nu=0,1, \ldots$, $[(p-2 k) / 2]$.

Remarks 9.1. (i) One may consider third and higher order tensorial traces. However, it is easily shown, just as with limiting traces in Proposition 3.4, that if $f_{p}$ has all of its first and second order tensorial traces, then the third order tensorial traces of $f_{p}$ also exist and are consistent.

(ii) Everything said in Definition 9.3 and in part (i) of this remark can be repeated with the tensorial traces $\operatorname{Tr}_{t}^{k} f_{p}$ replaced by $\operatorname{Tr}^{k} f_{p}$.

Our second corollary of Theorem 9.1 tells us that if $f_{p}$ has all of its first and second order tensorial traces, then they will necessarily be consistent.

Corollary 9.3. Let $f_{p} \in L_{s}^{2}\left(\mathbb{R}_{+}^{p}\right)$ and suppose tht $f_{p}$ has all of its first and second order tensorial traces. Then for nonnegative integers $k$ and $\nu$ such that $0 \leq$ $k+\nu \leq[p / 2]$,

$$
\operatorname{Tr}_{t}^{\nu}\left[\operatorname{Tr}_{t}^{k} f_{p}\right]=\operatorname{Tr}_{t}^{k+\nu} f_{p} \text {. }
$$

Proof. By Theorem 9.1, all the first and second order iterated traces exist and equal the corresponding tensorial traces. Applying this fact and formula (9.5), we obtain (9.13) as follows:

$$
\operatorname{Tr}_{t}^{\nu}\left[\operatorname{Tr}_{t}^{k} f_{p}\right]=\operatorname{Tr}_{i}^{\nu}\left[\operatorname{Tr}_{i}^{k} f_{p}\right]=\operatorname{Tr}_{i}^{k+\nu} f_{p}=\operatorname{Tr}_{t}^{k+\nu} f_{p}
$$

Since the existence of $\operatorname{Tr}^{k} f_{p}$ implies the existence of $\operatorname{Tr}_{t}^{k} f_{p}$ and equality, Corollary 9.3 immediately yields the following result.

Corollary 9.4. Let $f_{p} \in L_{s}^{2}\left(\mathbb{R}_{+}^{p}\right)$ and suppose that $f_{p}$ has all of its first and second order traces. Then for nonnegative integers $k$ and $\nu$ such that $0 \leq k+\nu \leq$ $[p / 2]$,

$$
\operatorname{Tr}^{\nu}\left[\operatorname{Tr}^{k} f_{p}\right]=\operatorname{Tr}^{k+\nu} f_{p} .
$$

Proposition 9.4. Let $f_{p} \in L_{s}^{2}\left(\mathbb{R}_{+}^{p}\right)$ and let $1 \leq k \leq[p / 2]$. Given any CONS $\left(\phi_{i}\right)$ for $L_{s}^{2}\left(\mathbb{R}_{+}\right)$, let

$$
f_{p}=\sum_{i_{1}, \ldots, i_{p}=1}^{\infty} a_{i_{1}, \ldots, i_{p}} \phi_{i_{1}} \otimes \cdots \otimes \phi_{i_{p}}
$$

be the usual expansion of $f_{p}$ in terms of the CONS $\left(\phi_{i_{1}} \otimes \cdots \otimes \phi_{i_{p}}\right)$ for $L_{s}^{2}\left(\mathbb{R}_{+}^{p}\right)$. Suppose that $\operatorname{Tr}_{t}^{k} f_{p}$ exists.

Then for every $i_{2 k+1}, \ldots, i_{p}$,

$$
\sum_{j_{1}, \ldots, j_{k}=1}^{\infty} \mid a_{j_{1}, j_{1}, \ldots, j_{k}, j_{k}, i_{2 k+1}, \ldots, i_{p} \mid<\infty,}
$$


and

$$
\sum_{i_{2 k+1}, \ldots, i_{p}=1}^{\infty}\left(\sum_{j_{1}, \ldots, j_{k}=1}^{\infty} a_{j_{1}, j_{1}, \ldots, j_{k}, j_{k}, i_{2 k+1}, \ldots, i_{p}}\right)^{2}<\infty
$$

Further, we have the formula

$$
\begin{aligned}
\operatorname{Tr}_{t}^{k} f_{p}= & \sum_{i_{2 k+1}, \ldots, i_{p}=1}^{\infty} \\
& \cdot\left(\sum_{j_{1}, \ldots, j_{k}=1}^{\infty} a_{j_{1}, j_{1}, \ldots, j_{k}, j_{k}, i_{2 k+1}, \ldots, i_{p}}\right) \phi_{i_{2 k+1}} \otimes \cdots \otimes \phi_{i_{p}} .
\end{aligned}
$$

Proof. Using Proposition 9.2 and then (9.15) we can write

$$
\begin{array}{r}
\operatorname{Tr}_{t}^{k} f_{p}=\sum_{j_{1}, \ldots, j_{k}=1}^{\infty} \int_{\mathbf{R}_{+}^{k} \times \mathbf{R}_{+}^{k}} f_{p}\left(s_{1}, \ldots, s_{2 k}, s_{2 k+1}, \ldots, s_{p}\right) \phi_{j_{1}}\left(s_{1}\right) \phi_{j_{1}}\left(s_{2}\right) \cdots \\
\cdot \phi_{j_{k}}\left(s_{2 k-1}\right) \phi_{j_{k}}\left(s_{2 k}\right) d s_{1} \cdots \cdot d s_{2 k} \\
=\sum_{j_{1}, \ldots, j_{k}=1}^{\infty} \int_{\mathbf{R}_{+}^{k} \times \mathbf{R}_{+}^{k}}\left[\sum_{i_{1}, \ldots, i_{p}=1}^{\infty} a_{i_{1}, \ldots, i_{p}} \phi_{i_{1}} \otimes \cdots \otimes \phi_{i_{p}}\right] \phi_{j_{1}}\left(s_{1}\right) \phi_{j_{1}}\left(s_{2}\right) \cdots \\
\cdot \phi_{j_{k}}\left(s_{2 k-1}\right) \phi_{j_{k}}\left(s_{2 k}\right) d s_{1} \cdots d s_{2 k}
\end{array}
$$

where the sum over the $j$ 's converges unconditionally (i.e., as an unordered sum). Now the inner sum in the third expression in (9.19) converges in $L_{s}^{2}\left(\mathbb{R}_{+}^{p}\right)$ norm and so the integral operators associated with the partial sums converge in Hilbert-Schmidt norm to the integral operator associated with $f_{p}$ and so certainly converge in operator norm and hence in the strong operator topology. Thus we can write

$$
\begin{gathered}
\operatorname{Tr}_{t}^{k} f_{p}=\sum_{j_{1}, \ldots, j_{k}=1}^{\infty}\left\{\sum_{i_{1}, \ldots, i_{p}=1}^{\infty} \int_{\mathbf{R}_{+}^{k} \times \mathbf{R}_{+}^{k}} a_{i_{1}, \ldots, i_{p}} \phi_{i_{1}}\left(s_{1}\right) \phi_{j_{1}}\left(s_{1}\right) \phi_{i_{2}}\left(s_{2}\right) \phi_{j_{1}}\left(s_{2}\right) \cdots\right. \\
\left.\cdot \phi_{i_{2 k-1}}\left(s_{2 k-1}\right) \phi_{j_{k}}\left(s_{2 k-1}\right) \phi_{i_{2 k}}\left(s_{2 k}\right) \phi_{j_{k}}\left(s_{2 k}\right) \phi_{i_{2 k+1}} \otimes \cdots \otimes \phi_{i_{p}} d s_{1} \cdots d s_{2 k}\right\} \\
=\sum_{j_{1}, \ldots, j_{k}=1}^{\infty}\left\{\sum_{i_{1}, \ldots, i_{p}=1}^{\infty} a_{i_{1}, \ldots, i_{p}}\left(\phi_{i_{1}}, \phi_{j_{1}}\right)\left(\phi_{i_{2}}, \phi_{j_{1}}\right) \cdots\right. \\
=\sum_{j_{1}, \ldots, j_{k}=1}^{\infty}\left\{\sum_{\left.\left.i_{2 k-1}, \phi_{j_{k}}\right)\left(\phi_{i_{2 k}}, \phi_{j_{k}}\right) \cdot \phi_{i_{2 k+1}} \otimes \cdots \otimes \phi_{i_{p}}\right\}}^{\infty} a_{j_{1}, j_{1}, \ldots, j_{k}, j_{k}, i_{2 k+1}, \ldots, i_{p}} \phi_{i_{2 k+1}} \otimes \cdots \otimes \phi_{i_{p}}\right\}
\end{gathered}
$$

Since norm convergence implies weak convergence we see from (9.20) that 
for any (temporarily fixed) $i_{2 k+1}^{\prime}, \ldots, i_{p}^{\prime}$,

$$
\begin{aligned}
& \left(\operatorname{Tr}_{t}^{k} f_{p}, \phi_{i_{2 k+1}^{\prime}} \otimes \cdots \otimes \phi_{i_{p}^{\prime}}\right)_{L_{s}^{2}\left(\mathbb{R}_{+}^{p-2 k}\right)} \\
& =\sum_{j_{1}, \ldots, j_{k}=1}^{\infty}\left\{\sum_{i_{2 k+1}, \ldots, i_{p}=1}^{\infty} a_{j_{1}, j_{1}, \ldots, j_{k}, j_{k}, i_{2 k+1}, \ldots, i_{p}}\left(\phi_{i_{2 k+1}}, \phi_{i_{2 k+1}^{\prime}}\right)\right. \\
& \left.\cdots \cdots\left(\phi_{i_{p}}, \phi_{i_{p}^{\prime}}\right)\right\} \\
& \quad=\sum_{j_{1}, \ldots, j_{k}=1}^{\infty} a_{j_{1}, j_{1}, \ldots, j_{k}, j_{k}, i_{2 k+1}^{\prime}, \ldots, i_{p}^{\prime} .}
\end{aligned}
$$

Hence the last series in (9.21) converges; in fact, since the sum over the $j$ 's in (9.21) and therefore in (9.20) converges unconditionally, we see that the series in $(9.21)$ converges unconditionally and so absolutely for every $i_{2 k+1}^{\prime}, \ldots, i_{p}^{\prime}$. Inequality (9.16) now follows. Further (9.21) gives us the coefficients of the expansion of $\operatorname{Tr}_{t}^{k} f_{p}$ in terms of the CONS $\left(\phi_{i_{2 k+1}} \otimes \cdots \otimes \phi_{i_{p}}\right)$ for $L_{s}^{2}\left(\mathbb{R}_{+}^{p-2 k}\right)$. Inequality (9.17) and equality (9.18) now follow immediately.

The expansion (9.20) for $\operatorname{Tr}_{t}^{k} f_{p}$ will be useful to us further on and so we state this result as a corollary of the proof of the preceding proposition.

Corollary 9.5. Let the hypotheses of Proposition 9.4 be satisfied. Then we have

$$
\operatorname{Tr}_{t}^{k} f_{p}=\sum_{j_{1}, \ldots, j_{k}=1}^{\infty}\left\{\sum_{i_{2 k+1}, \ldots, i_{p}=1}^{\infty} a_{j_{1}, j_{1}, \ldots, j_{k}, j_{k}, i_{2 k+1}, \ldots, i_{p}} \phi_{i_{2 k+1}} \otimes \cdots \otimes \phi_{i_{p}}\right\}
$$

where the series in the $j$ 's converges unconditionally in $L_{s}^{2}\left(\mathbb{R}_{+}^{p-2 k}\right)$.

Recalling that the existence of $\operatorname{Tr}^{k} f_{p}$ implies the existence of $\operatorname{Tr}_{t}^{k} f_{p}$, we immediately obtain the following corollary of Proposition 9.4 and Corollary 9.5 .

Corollary 9.6. Let the hypotheses of Proposition 9.4 be satisfied except that we assume the existence of $\operatorname{Tr}^{k} f_{p}$ rather than $\operatorname{Tr}_{t}^{k} f_{p}$. Then the conclusions of Proposition 9.4 and Corollary 9.5 hold where in formulas (9.18) and (9.22), $\operatorname{Tr}_{t}^{k} f_{p}$ is replaced by $\operatorname{Tr}^{k} f_{p}$.

Remark 9.2. Since the iterated 1-trace precisely coincides with the tensorial 1trace, Proposition 9.4 and Corollary 9.5 yield some information about $\operatorname{Tr}_{i}^{1} f_{p}$, $\operatorname{Tr}_{i}^{2} f_{p}, \ldots, \operatorname{Tr}_{i}^{k} f_{p}$ assuming that $\operatorname{Tr}_{i}^{k} f_{p}$ exists. As usual, let $f_{p} \in L_{s}^{2}\left(\mathbb{R}_{+}^{p}\right)$ and let $1 \leq k \leq[p / 2]$. Further, given any $\operatorname{CONS}\left(\phi_{i}\right)$ for $L_{s}^{2}\left(\mathbb{R}_{+}\right)$, let $(9.15)$ be the expansion of $f_{p}$ in terms of the CONS $\left(\phi_{i_{1}} \otimes \cdots \otimes \phi_{i_{p}}\right)$ for $L_{s}^{2}\left(\mathbb{R}_{+}^{p}\right)$. If $\operatorname{Tr}_{i}^{1} f_{p}$ exists, then for every $i_{3}, \ldots, i_{p}$,

$$
\sum_{j_{1}=1}^{\infty}\left|a_{j_{1}, j_{1}, i_{3}, \ldots, i_{p}}\right|<\infty
$$


Also

$$
\sum_{i_{3}, \ldots, i_{p}=1}^{\infty}\left(\sum_{j_{1}=1}^{\infty} a_{j_{1}, j_{1}, i_{3}, \ldots, i_{p}}\right)^{2}<\infty,
$$

and we have the formulas

$$
\begin{aligned}
\operatorname{Tr}_{i}^{1} f_{p} & =\sum_{i_{3}, \ldots, i_{p}=1}^{\infty}\left(\sum_{j_{1}=1}^{\infty} a_{j_{1}, j_{1}, i_{3}, \ldots, i_{p}}\right) \phi_{i_{3}} \otimes \cdots \otimes \phi_{i_{p}} \\
& =\sum_{j_{1}=1}^{\infty}\left(\sum_{i_{3}, \ldots, i_{p}=1}^{\infty} a_{j_{1}, j_{1}, i_{3}, \ldots, i_{p}} \phi_{i_{3}} \otimes \cdots \otimes \phi_{i_{p}}\right)
\end{aligned}
$$

where the second expression in (9.25) is the expansion of $\operatorname{Tr}_{i}^{1} f_{p}$ in terms of the CONS $\left(\phi_{i_{3}} \otimes \cdots \otimes \phi_{i_{p}}\right)$ for $L_{s}^{2}\left(\mathbb{R}_{+}^{p-2}\right)$ and where the third expression in (9.25) is an unconditionally convergent series in $j_{1}$ in the space $L_{s}^{2}\left(\mathbb{R}_{+}^{p-2}\right)$.

Now if $\operatorname{Tr}_{i}^{2} f_{p}$ exists, we begin with the orthogonal expansion,

$$
\operatorname{Tr}_{i}^{1} f_{p}=\sum_{i_{3}, \ldots, i_{p}=1}^{\infty} a_{i_{3}, \ldots, i_{p}}^{(1)} \phi_{i_{3}} \otimes \cdots \otimes \phi_{i_{p}},
$$

where

$$
a_{i_{3}, \ldots, i_{p}}^{(1)}:=\sum_{j_{1}=1}^{\infty} a_{j_{1}, j_{1}, i_{3}, \ldots, i_{p}}
$$

and apply Proposition 9.4 and Corollary 9.5 again. Doing this, we see that

$$
\sum_{j_{2}=1}^{\infty}\left|\sum_{j_{1}=1}^{\infty} a_{j_{1}, j_{1}, j_{2}, j_{2}, i_{5}, \ldots, i_{p}}\right|=\sum_{j_{2}=1}^{\infty}\left|a_{j_{2}, j_{2}, i_{5}, \ldots, i_{p}}^{(1)}\right|<\infty,
$$

for every $i_{5}, \ldots, i_{p}$, and that

$$
\begin{gathered}
\sum_{i_{5}, \ldots, i_{p}=1}\left[\sum_{j_{2}=1}^{\infty}\left(\sum_{j_{1}=1}^{\infty} a_{j_{1}, j_{1}, j_{2}, j_{2}, i_{5}, \ldots, i_{p}}\right)\right]^{2} \\
=\sum_{i_{5}, \ldots, i_{p}=1}^{\infty}\left[\sum_{j_{2}=1}^{\infty} a_{j_{2}, j_{2}, i_{5}, \ldots, i_{p}}^{(1)}\right]^{2}<\infty .
\end{gathered}
$$

Further, we have the expansion

$$
\begin{aligned}
\operatorname{Tr}_{i}^{2} f_{p} & =\sum_{i_{5}, \ldots, i_{p}=1}^{\infty}\left[\sum_{j_{2}=1}^{\infty}\left(\sum_{j_{1}=1}^{\infty} a_{j_{1}, j_{1}, j_{2}, j_{2}, i_{5}, \ldots, i_{p}}\right)\right] \phi_{i_{5}} \otimes \cdots \otimes \phi_{i_{p}} \\
& =\sum_{i_{5}, \ldots, i_{p}=1}^{\infty}\left[\sum_{j_{2}=1}^{\infty} a_{j_{2}, j_{2}, i_{5}, \ldots, i_{p}}^{(1)}\right] \phi_{i_{5}} \otimes \cdots \otimes \phi_{i_{p}}
\end{aligned}
$$


where (9.30) is the orthogonal expansion of $\operatorname{Tr}_{i}^{2} f_{p}$ in terms of the CONS $\left(\phi_{i_{s}} \otimes\right.$ $\left.\cdots \otimes \phi_{i_{p}}\right)$ for $L_{s}^{2}\left(\mathbb{R}_{+}^{p-4}\right)$. As the final part of the second stage, we have

$$
\begin{aligned}
\operatorname{Tr}_{i}^{2} f_{p} & =\sum_{j_{2}=1}^{\infty}\left\{\sum_{i_{5}, \ldots, i_{p}=1}^{\infty}\left(\sum_{j_{1}=1}^{\infty} a_{j_{1}, j_{1}, j_{2}, j_{2}, i_{5}, \ldots, i_{p}}\right) \phi_{i_{5}} \otimes \cdots \otimes \phi_{i_{p}}\right\} \\
& =\sum_{j_{2}=1}^{\infty}\left\{\sum_{i_{5}, \ldots, i_{p}=1}^{\infty} a_{j_{2}, j_{2}, i_{5}, \ldots, i_{p}}^{(1)} \phi_{i_{5}} \otimes \cdots \otimes \phi_{i_{p}}\right\},
\end{aligned}
$$

where the series in $j_{2}$ converges unconditionally in the space $L^{2}\left(\mathbb{R}_{+}^{p-4}\right)$.

The process can be continued inductively as long as the corresponding iterated traces exist.

The limiting $k$-trace was the crucial one earlier in this paper, but we have had little to say about it so far in this section. We now begin to relate $\overrightarrow{\operatorname{Tr}}^{k} f_{p}$ to the other $k$-traces. Our next result is the key.

Theorem 9.2. Let $f_{p} \in L_{s}^{2}\left(\mathbb{R}_{+}^{p}\right)$ and let $1 \leq k \leq[p / 2]$. If $\operatorname{Tr}_{t}^{k} f_{p}$ exists then $\overrightarrow{\mathrm{Tr}}^{k} f_{p}$ exists and

$$
\overrightarrow{\operatorname{Tr}}^{k} f_{p}=\operatorname{Tr}_{t}^{k} f_{p} \text {. }
$$

Proof. Let $\left(\phi_{i}\right)$ be any CONS for $L_{s}^{2}\left(\mathbb{R}_{+}\right)$. Let

$$
f_{p}=\sum_{i_{1}, \ldots, i_{p}=1}^{\infty} a_{i_{1}, \ldots, i_{p}} \phi_{i_{1}} \otimes \cdots \otimes \phi_{i_{p}} .
$$

In dealing with the limiting $k$-trace, we should begin, according to Definition 3.2 , by considering the truncated series

$$
f_{p,\left(\phi_{i}\right)}^{N}=\sum_{i_{i}, \ldots, i_{p}=1}^{N} a_{i_{1}, \ldots, i_{p}} \phi_{i_{1}} \otimes \cdots \otimes \phi_{i_{p}}
$$

By Proposition 3.1, $\operatorname{Tr}^{k} f_{p,\left(\phi_{i}\right)}^{N}$ exists and is given by

$$
\operatorname{Tr}^{k} f_{p,\left(\phi_{i}\right)}^{N}=\sum_{i_{2 k+1}, \ldots, i_{p}=1}^{N}\left(\sum_{j_{1}, \ldots, j_{k}=1}^{N} a_{j_{1}, j_{1}, \ldots, j_{k}, j_{k}, i_{2 k+1}, \ldots, i_{p}}\right) \phi_{i_{2 k+1}} \otimes \cdots \otimes \phi_{i_{p}} .
$$

In order to show that $\overrightarrow{\operatorname{Tr}}^{k} f_{p}$ exists and equals $\operatorname{Tr}_{t}^{k} f_{p}$, it suffices to show that

$$
\left\|\operatorname{Tr}^{k} f_{p,\left(\phi_{i}\right)}^{N}-\operatorname{Tr}_{t}^{k} f_{p}\right\|_{L_{s}^{2}\left(\mathbf{R}_{+}^{p-2 k}\right)}^{2} \rightarrow 0
$$


as $N \rightarrow \infty$. Now by Proposition 9.4 we know that

(9.37) $\operatorname{Tr}_{t}^{k} f_{p}=\sum_{i_{2 k+1}, \ldots, i_{p}=1}^{\infty}\left(\sum_{j_{1}, \ldots, j_{k}=1}^{\infty} a_{j_{1}, j_{1}, \ldots, j_{k}, j_{k}, i_{2 k+1}, \ldots, i_{p}}\right) \phi_{i_{2 k+1}} \otimes \cdots \otimes \phi_{i_{p}}$

where, furthermore, the inequalities $(9.16)$ and (9.17) hold. From the orthogonal expansion (9.37), we see that as $N \rightarrow \infty$,

$$
\begin{array}{r}
\| \sum_{i_{2 k+1}, \ldots, i_{p}=1}^{N}\left(\sum_{j_{1}, \ldots, j_{k}=1}^{\infty} a_{j_{1}, j_{1}, \ldots, j_{k}, j_{k}, i_{2 k+1}, \ldots, i_{p}}\right) \phi_{i_{2 k+1}} \\
\otimes \cdots \otimes \phi_{i_{p}}-\operatorname{Tr}_{t}^{k} f_{p} \|^{2} \rightarrow 0 .
\end{array}
$$

In light of (9.35) and (9.38), to show (9.36) it suffices to show that

$$
\sum_{i_{2 k+1}, \ldots, i_{p}=1}^{N}\left(\sum_{\left(j_{1}, \ldots, j_{k}\right) \in\left(J_{N}^{k}\right)^{c}} a_{j_{1}, j_{1}, \ldots, j_{k}, j_{k}, i_{2 k+1}, \ldots, i_{p}}\right)^{2} \rightarrow 0
$$

as $N \rightarrow \infty$ where $J_{N}:=\{1, \ldots, N\}$. Further, to show (9.39) it clearly suffices to show that as $N \rightarrow \infty$,

$$
\sum_{i_{2 k+1}, \ldots, i_{p}=1}^{\infty}\left(\sum_{\left(j_{1}, \ldots, j_{k}\right) \in\left(J_{N}^{k}\right)^{c}} a_{j_{1}, j_{1}, \ldots, j_{k}, j_{k}, i_{2 k+1}, \ldots, i_{p}}\right)^{2} \rightarrow 0
$$

Now given integers $N$ and $m$ and regarding $N$ as fixed (for now), let

$$
A_{N, m}:=J_{N+m}^{k} \backslash J_{N}^{k}
$$

Note that the sequence of sets $\left\{A_{N, m}: m=1,2, \ldots\right\}$ is increasing and

$$
\left(J_{N}^{k}\right)^{c}=\bigcup_{m=1}^{\infty} A_{N, m}
$$

From these observations, from (9.16) and from Fatou's Lemma, we see that 


$$
\begin{gathered}
\sum_{i_{2 k+1}, \ldots, i_{p}=1}^{\infty}\left(\sum_{\left(j_{1}, \ldots, j_{k}\right) \in\left(J_{N}^{k}\right)^{c}} a_{j_{1}, j_{1}, \ldots, j_{k}, j_{k}, i_{2 k+1}, \ldots, i_{p}}\right)^{2} \\
=\sum_{i_{2 k+1}, \ldots, i_{p}=1}^{\infty} \lim _{m \rightarrow \infty}\left(\sum_{\left(j_{1}, \ldots, j_{k}\right) \in A_{N, m}} a_{j_{1}, j_{1}, \ldots, j_{k}, j_{k}, i_{2 k+1}, \ldots, i_{p}}\right)^{2} \\
\leq \liminf _{m \rightarrow \infty} \sum_{i_{2 k+1}, \ldots, i_{p}=1}^{\infty}\left(\sum_{\left(j_{1}, \ldots, j_{k}\right) \in A_{N, m}} a_{j_{1}, j_{1}, \ldots, j_{k}, j_{k}, i_{2 k+1}, \ldots, i_{p}}\right)^{2} \\
=\liminf _{m \rightarrow \infty}\left\|\sum_{i_{2 k+1}, \ldots, i_{p}=1}^{\infty}\left(\sum_{\left(j_{1}, \ldots, j_{k}\right) \in A_{N, m}} a_{j_{1}, j_{1}, \ldots, j_{k}, j_{k}, i_{2 k+1}, \ldots, i_{p}}\right)^{2}\right\|_{i_{2 k+1}} \otimes \ldots \otimes \phi_{i_{p}} \|^{2} \\
=\liminf _{n \rightarrow \infty} \| \sum_{\left(j_{1}, \ldots, j_{k}\right) \in A_{N, m}}\left(\sum_{i_{2 k+1}, \ldots, i_{p}=1}^{\infty} a_{j_{1}, j_{1}, \ldots, j_{k}, j_{k}, i_{2 k+1}, \ldots, i_{p}}\right. \\
\left.\cdot \phi_{i_{2 k+1}} \otimes \ldots \otimes \phi_{i_{p}}\right) \|^{2} .
\end{gathered}
$$

But the series in the $j$ 's in (9.22) of Corollary 9.5 converges unconditionally in $L_{s}^{2}\left(\mathbb{R}_{+}^{p-2 k}\right)$. From this we see that as $m \rightarrow \infty$

$$
\begin{aligned}
& \sum_{\left(j_{1}, \ldots, j_{k}\right) \in A_{N, m}}\left(\sum_{i_{2 k+1}, \ldots, i_{p}=1}^{\infty} a_{j_{1}, j_{1}, \ldots, j_{k}, j_{k}, i_{2 k+1}, \ldots, i_{p}} \phi_{i_{2 k+1}} \otimes \cdots \otimes \phi_{i_{p}}\right) \\
& \rightarrow \sum_{\left(j_{1}, \ldots, j_{k}\right) \in\left(J_{N}^{k}\right)^{c}}\left(\sum_{i_{2 k+1}, \ldots, i_{p}=1}^{\infty} a_{j_{1}, j_{1}, \ldots, j_{k}, j_{k}, i_{2 k+1}, \ldots, i_{p} \phi_{i_{2 k+1}} \otimes \cdots \otimes \phi_{i_{p}}}\right)
\end{aligned}
$$

in the norm on $L^{2}\left(\mathbb{R}_{+}^{p-2 k}\right)$. Using (9.44) to calculate the limit (which equals the limit infimum) in the last expression in (9.43), we obtain from (9.43) (9.45)

$$
\begin{aligned}
& \sum_{i_{2 k+1}, \ldots, i_{p}=1}^{\infty}\left(\sum_{\left(j_{1}, \ldots, j_{k}\right) \in\left(J_{N}^{k}\right) c} a_{j_{1}, j_{1}, \ldots, j_{k}, j_{k}, i_{2 k+1}, \ldots, i_{p}}\right)^{2} \\
& \leq \| \sum_{\left(j_{1}, \ldots, j_{k}\right) \in\left(J_{N}^{k}\right) c}\left(\sum_{i_{2 k+1}, \ldots, i_{p}=1}^{\infty} a_{\left.j_{1}, j_{1}, \ldots, j_{k}, j_{k}, i_{2 k+1}, \ldots, i_{p} \phi_{i_{2 k+1}} \otimes \cdots \otimes \phi_{i_{p}}\right) \|^{2} \rightarrow 0}\right.
\end{aligned}
$$

where the convergence to 0 in (9.45) can be seen by turning again to the series in (9.22) and noting that it converges unconditionally in the $j$ 's in the norm on $L^{2}\left(\mathbb{R}_{+}^{p-2 k}\right)$. This establishes $(9.40)$ and completes the proof. 

9.1.

Our first corollary follows immediately from Theorem 9.2 and Proposition

Corollary 9.7. Let $f_{p} \in L_{s}^{2}\left(\mathbb{R}_{+}^{p}\right)$ and let $1 \leq k \leq[p / 2]$. If $\operatorname{Tr}^{k} f_{p}$ exists, then $\operatorname{Tr}_{t}^{k} f_{p}$ and $\overrightarrow{\operatorname{Tr}}^{k} f_{p}$ both exist and

$$
\overrightarrow{\operatorname{Tr}}^{k} f_{p}=\operatorname{Tr}_{t}^{k} f_{p}=\operatorname{Tr}^{k} f_{p}
$$

Applying Theorem 9.1 and Corollary 9.2 as well as Theorem 9.2, we obtain our next corollary.

Corollary 9.8. Let $f_{p} \in L_{s}^{2}\left(\mathbb{R}_{+}^{p}\right)$ and suppose that $\operatorname{Tr}_{t}^{1} f_{p}, \ldots, \operatorname{Tr}_{t}^{\nu} f_{p}$ all exist where $1 \leq \nu \leq[p / 2]$. Then all of $\operatorname{Tr}_{i}^{1} f_{p}, \ldots, \operatorname{Tr}_{i}^{\nu} f_{p}$ and $\overrightarrow{\operatorname{Tr}}^{1} f_{p}, \ldots, \overrightarrow{\operatorname{Tr}}^{\nu} f_{p}$ exist and, for every $k$ such that $1 \leq k \leq \nu$,

$$
\overrightarrow{\operatorname{Tr}}^{k} f_{p}=\operatorname{Tr}_{i}^{k} f_{p}=\operatorname{Tr}_{t}^{k} f_{p} .
$$

If we assume instead that $\operatorname{Tr}^{1} f_{p}, \ldots, \operatorname{Tr}^{\nu} f_{p}$ all exist, then all of $\operatorname{Tr}_{t}^{1} f_{p}, \ldots$, $\operatorname{Tr}_{t}^{\nu} f_{p}, \operatorname{Tr}_{i}^{1} f_{p}, \ldots, \operatorname{Tr}_{i}^{\nu} f_{p}$ and $\overrightarrow{\operatorname{Tr}}^{1} f_{p}, \ldots, \overrightarrow{\operatorname{Tr}}^{\nu} f_{p}$ exist and, for every $k$ such that $1 \leq k \leq \nu$,

$$
\overrightarrow{\operatorname{Tr}}^{k} f_{p}=\operatorname{Tr}_{i}^{k} f_{p}=\operatorname{Tr}_{t}^{k} f_{p}=\operatorname{Tr}^{k} f_{p} .
$$

Corollary 9.8 allows us to strengthen Corollary 9.3.

Corollary 9.9. Let $f_{p} \in L_{s}^{2}\left(\mathbb{R}_{+}^{p}\right)$ and suppose that $f_{p}$ has all its first and second order tensorial traces. Then for nonnegative integers $k$ and $\nu$ such that $0 \leq$ $k+\nu \leq[p / 2]$, we have not only (9.13) as before, but, in addition, all of the first and second order limiting traces exist and

$$
\overrightarrow{\operatorname{Tr}}^{\nu}\left[\overrightarrow{\operatorname{Tr}}^{k} f_{p}\right]=\overrightarrow{\operatorname{Tr}}^{k+\nu} f_{p}
$$

Proof. The existence of all the first and second order limiting and iterated traces and their equality follows from Corollary 9.8. Formula (9.49) is now a consequence of (9.13).

Remark 9.3. If $f_{p} \in L_{s}^{2}\left(\mathbb{R}_{+}^{p}\right)$ has all of its first and second order traces, then it has all of its first and second order tensorial traces and so the conclusions of Corollary 9.9 hold.

We give one more corollary of Theorem 9.2.

Corollary 9.10. Let $f_{p} \in L_{s}^{2}\left(\mathbb{R}_{+}^{p}\right)$ and suppose that $\operatorname{Tr}_{t}^{k} f_{p}, \operatorname{Tr}_{t}^{\nu}\left[\operatorname{Tr}_{t}^{k} f_{p}\right]$ and $\operatorname{Tr}_{t}^{k+\nu} f_{p}$ exist and (9.13) holds where $k$ and $\nu$ are fixed nonnegative integers such that $0 \leq k+\nu \leq[p / 2]$. Then $\overrightarrow{\operatorname{Tr}}^{k} f_{p}, \overrightarrow{\operatorname{Tr}}^{\nu}\left[\overrightarrow{\operatorname{Tr}}^{k} f_{p}\right]$ and $\overrightarrow{\operatorname{Tr}}^{k+\nu} f_{p}$ exist and (9.49) holds.

In Proposition 3.1 we saw that $\operatorname{Tr}^{k} f_{p}$ exists for functions $f_{p}$ possessing finite tensorial expansions. The result immediately below goes considerably further.

Theorem 9.3. Let $f_{p} \in L_{s}^{2}\left(\mathbb{R}_{+}^{p}\right)$ and suppose that there exists a CONS $\left(\phi_{i}\right)$ for $L^{2}\left(\mathbb{R}_{+}\right)$such that the coefficients $\left(a_{i_{1}}, \ldots, i_{p}\right)$ in the expansion for $f_{p}, f_{p}=$ $\sum_{i_{1}, \ldots, i_{p}=1}^{\infty} a_{i_{1}, \ldots, i_{p}} \phi_{i_{1}} \otimes \cdots \otimes \phi_{i_{p}}$, belong to $l_{1}$. 
Then for $0 \leq k \leq[p / 2], \operatorname{Tr}^{k} f_{p}$ exists and is given by

(9.50) $\operatorname{Tr}^{k} f_{p}=\sum_{i_{2 k+1}, \ldots, i_{p}=1}^{\infty}\left(\sum_{j_{1}, \ldots, j_{k}=1}^{\infty} a_{j_{1}, j_{1}, \ldots, j_{k}, j_{k}, i_{2 k+1}, \ldots, i_{p}}\right) \phi_{i_{2 k+1}} \otimes \cdots \otimes \phi_{i_{p}}$.

Proof. Let $\left(e_{j}\right)$ be any CONS for $L^{2}\left(\mathbb{R}_{+}^{k}\right)$. It suffices to show that (9.51)

$$
\begin{aligned}
& \sum_{j=1}^{\infty} \int_{\mathbb{R}_{+}^{k} \times \mathbb{R}_{+}^{k}} f_{p}\left(s_{1}, \ldots, s_{k}, s_{k+1}, \ldots, s_{2 k}, s_{2 k+1}, \ldots, s_{p}\right) \\
& \cdot e_{j}\left(s_{1}, \ldots, s_{k}\right) e_{j}\left(s_{k+1}, \ldots, s_{2 k}\right) d s_{1} \cdots d s_{k} d s_{k+1} \cdots d s_{2 k} \\
&=\sum_{i_{2 k+1}, \ldots, i_{p}=1}^{\infty}\left(\sum_{j_{1}, \ldots, j_{k}=1}^{\infty} a_{j_{1}, j_{1}, \ldots, j_{k}, j_{k}, i_{2 k+1}, \ldots, i_{p}}\right) \phi_{i_{2 k+1}} \otimes \cdots \otimes \phi_{i_{p}}
\end{aligned}
$$

where the series in $j$ converges in the $L^{2}\left(\mathbb{R}_{+}^{p-2 k}\right)$ norm. For the moment, we fix $j$ and consider the integral on the left-hand side of (9.51) with $f_{p}$ replaced by its series expansion:

$$
\begin{gathered}
\int_{\mathbb{R}_{+}^{k} \times \mathbb{R}_{+}^{k}}\left[\sum_{i_{1}, \ldots, i_{p}=1}^{\infty} a_{i_{1}, \ldots, i_{p}} \phi_{i_{1}}\left(s_{1}\right) \cdots \cdot \phi_{i_{k}}\left(s_{k}\right) \cdot \phi_{i_{k+1}}\left(s_{k+1}\right) \cdots\right. \\
\left.\cdot \phi_{i_{2 k}}\left(s_{2 k}\right) \phi_{i_{2 k+1}}\left(s_{2 k+1}\right) \cdots \cdot \phi_{i_{p}}\left(s_{p}\right)\right] \\
\cdot e_{j}\left(s_{1}, \ldots, s_{k}\right) e_{j}\left(s_{k+1}, \ldots, s_{2 k}\right) d s_{1} \cdots d s_{k} \cdot d s_{k+1} \cdots \cdot d s_{2 k} .
\end{gathered}
$$

Now the sequence of partial sums

$$
\sum_{i_{1}, \ldots, i_{p}=1}^{N_{1}, \ldots, N_{p}} a_{i_{1}, \ldots, i_{p}} \phi_{i_{1}} \otimes \cdots \otimes \phi_{i_{p}}
$$

is a sequence of $L_{s}^{2}\left(\mathbb{R}_{+}^{p}\right)$ kernels converging to the $L_{s}^{2}\left(\mathbb{R}_{+}^{p}\right)$ kernel $f_{p}$. It follows that the associated integral operators converge in Hilbert-Schmidt norm, hence in operator norm and so certainly in the strong operator topology. Thus the expression in (9.52) equals

$$
\begin{aligned}
& \sum_{i_{1}, \ldots, i_{p}=1}^{\infty} a_{i_{1}, \ldots, i_{p}} \int_{\mathbb{R}_{+}^{k} \times \mathbf{R}_{+}^{k}} \phi_{i_{1}}\left(s_{1}\right) \cdots \cdots \phi_{i_{k}}\left(s_{k}\right) \cdot e_{j}\left(s_{1}, \ldots, s_{k}\right) \phi_{i_{k+1}}\left(s_{k+1}\right) \cdot \ldots \\
& \quad \cdot \phi_{i_{2 k}}\left(s_{2 k}\right) e_{j}\left(s_{k+1}, \ldots, s_{2 k}\right) \phi_{i_{2 k+1}}\left(s_{2 k+1}\right) \cdots \cdots \phi_{i_{p}}\left(s_{p}\right) d s_{1} \cdots d s_{k} d s_{k+1} \cdots d s_{2 k} \\
& \quad=\sum_{i_{1}, \ldots, i_{p}=1}^{\infty} a_{i_{1}, \ldots, i_{p}}\left(\phi_{i_{1}} \otimes \cdots \otimes \phi_{i_{k}}, e_{j}\right)\left(\phi_{i_{k+1}} \otimes \cdots \otimes \phi_{i_{2 k}}, e_{j}\right) \phi_{i_{2 k+1}} \otimes \cdots \otimes \phi_{i_{p}} .
\end{aligned}
$$

Returning to the left-hand side of (9.51) and using our results so far we can 
write

(9.54)

$$
\begin{aligned}
& \sum_{j=1}^{\infty} \int_{\mathbb{R}_{+}^{k} \times \mathbb{R}_{+}^{k}} f_{p}\left(s_{1}, \ldots, s_{p}\right) e_{j}\left(s_{1}, \ldots, s_{k}\right) e_{j}\left(s_{k+1}, \ldots, s_{2 k}\right) d s_{1} \cdots d s_{2 k} \\
& \quad=\sum_{j=1}^{\infty} \sum_{i_{1}, \ldots, i_{p}=1}^{\infty} a_{i_{1}, \ldots, i_{p}}\left(\phi_{i_{1}} \otimes \cdots \otimes \phi_{i_{k}}, e_{j}\right)\left(\phi_{i_{k+1}} \otimes \cdots \otimes \phi_{i_{2 k}}, e_{j}\right) \phi_{i_{2 k+1}} \otimes \cdots \otimes \phi_{i_{p}} \\
& \quad=\sum_{i_{1}, \ldots, i_{p}=1}^{\infty} a_{i_{1}, \ldots, i_{p}}\left\{\sum_{j=1}^{\infty}\left(\phi_{i_{1}} \otimes \cdots \otimes \phi_{i_{k}}, e_{j}\right)\left(\phi_{i_{k+1}} \otimes \cdots \otimes \phi_{i_{2 k}}, e_{j}\right)\right\} \phi_{i_{2 k+1}} \otimes \cdots \otimes \phi_{i_{p}} \\
& \quad=\sum_{i_{1}, \ldots, i_{p}=1}^{\infty} a_{i_{1}, \ldots, i_{p}}\left(\phi_{i_{1}} \otimes \cdots \otimes \phi_{i_{k}}, \phi_{i_{k+1}} \otimes \cdots \otimes \phi_{i_{2 k}}\right) \phi_{i_{2 k+1}} \otimes \cdots \otimes \phi_{i_{p}} \\
& \quad=\sum_{i_{2 k+1}, \ldots, i_{p}=1}^{\infty}\left(\sum_{j_{1}, \ldots, j_{k}=1}^{\infty} a_{j_{1}, j_{1}, \ldots, j_{k}, j_{k}, i_{2 k+1}, \ldots, i_{p}}\right) \phi_{i_{2 k+1}} \otimes \cdots \otimes \phi_{i_{p}},
\end{aligned}
$$

where the last equality follows from the fact that the coefficients $\left(a_{i_{1}}, \ldots, i_{p}\right)$ are symmetric in the indices and in $l_{1}$. We will finish the proof by justifying the second equality in (9.54): The sequences $\left(\phi_{i_{1}} \otimes \cdots \otimes \phi_{i_{k}}, e_{j}\right)$ and $\left(\phi_{i_{k+1}} \otimes \cdots \otimes\right.$ $\left.\phi_{i_{2 k}}, e_{j}\right)$ are in $l_{2}$ as functions of $j$. Therefore

$$
\left|\left(\phi_{i_{1}} \otimes \cdots \otimes \phi_{i_{k}}, e_{j}\right)\right|\left|\left(\phi_{i_{k+1}} \otimes \cdots \otimes \phi_{i_{2 k}}, e_{j}\right)\right|
$$

is in $l_{1}$ and, in fact,

$$
\begin{aligned}
\sum_{j=1}^{\infty} \mid & \left(\phi_{i_{1}} \otimes \cdots \otimes \phi_{i_{k}}, e_{j}\right)||\left(\phi_{i_{k+1}} \otimes \cdots \otimes \phi_{i_{2 k}}, e_{j}\right) \mid \\
& \leq\left[\sum_{j=1}^{\infty}\left(\phi_{i_{1}} \otimes \cdots \otimes \phi_{i_{k}}, e_{j}\right)^{2}\right]^{1 / 2} \cdot\left[\sum_{j=1}^{\infty}\left(\phi_{i_{k+1}} \otimes \cdots \otimes \phi_{i_{2 k}}, e_{j}\right)^{2}\right]^{1 / 2} \\
& =\left\|\phi_{i_{1}} \otimes \cdots \otimes \phi_{i_{k}}\right\| \cdot\left\|\phi_{i_{k+1}} \otimes \cdots \otimes \phi_{i_{2 k}}\right\| \\
& =1 .
\end{aligned}
$$

Using (9.55) and the $l_{1}$-assumption on the coefficients $\left(a_{i_{1}}, \ldots, i_{p}\right)$ and thinking of the sums on both sides of the second equality in (9.54) as Bochner integrals, we can now apply the Fubini Theorem for Bochner integrals [4, Theorem 3.7.13] and obtain the desired equality.

We have already seen that the four $k$-traces are related to one another in various ways. The following corollary to Theorem 9.3 identifies, in terms of orthogonal expansions, a large class of $f_{p}$ 's for which all four $k$-traces exist and agree.

Corollary 9.11. Let $f_{p} \in L_{s}^{2}\left(\mathbb{R}_{+}^{p}\right)$ and suppose that there exists a CONS $\left(\phi_{i}\right)$ for $L^{2}\left(\mathbb{R}_{+}\right)$such that the coefficients $\left(a_{i_{1}}, \ldots, i_{p}\right)$ in the expansion for $f_{p}$,

$$
f_{p}=\sum_{i_{1}, \ldots, i_{p}=1}^{\infty} a_{i_{1}, \ldots, i_{p}} \phi_{i_{1}} \otimes \cdots \otimes \phi_{i_{p}},
$$

belong to $l_{1}$. Then for $0 \leq k \leq[p / 2]$, all four $k$-traces exist and we have

$$
\operatorname{Tr}^{k} f_{p}=\operatorname{Tr}_{t}^{k} f_{p}=\operatorname{Tr}_{i}^{k} f_{p}=\overrightarrow{\operatorname{Tr}}^{k} f_{p}
$$


Proof. By Theorem 9.3, $\operatorname{Tr}^{k} f_{p}$ exists for all $k, 0 \leq k \leq[p / 2]$. It follows from Corollary 9.2 and Theorem 9.2 that all four $k$-traces exist and (9.56) holds.

Under the assumptions of Theorem 9.3 (or Corollary 9.11), not only do all four $k$-traces exist and agree but also all the second order traces exist and agree and are consistent with the first order traces. This is the content of our last corollary.

Corollary 9.12. Suppose that the assumptions of Theorem 9.3 are satisfied. Then all of the first and second order traces of all four types exist, and, for any nonnegative integers $k$ and $\nu$ such that $0 \leq k+\nu \leq[p / 2]$, we have

$$
\begin{aligned}
\operatorname{Tr}^{k+\nu} f_{p} & =\operatorname{Tr}_{t}^{k+\nu} f_{p}=\operatorname{Tr}_{i}^{k+\nu} f_{p}=\overrightarrow{\operatorname{Tr}}^{k+\nu} f_{p}=\operatorname{Tr}^{k}\left[\operatorname{Tr}^{\nu} f_{p}\right] \\
& =\operatorname{Tr}_{t}^{k}\left[\operatorname{Tr}_{t}^{\nu} f_{p}\right]=\operatorname{Tr}_{i}^{k}\left[\operatorname{Tr}_{i}^{\nu} f_{p}\right]=\overrightarrow{\operatorname{Tr}}^{k}\left[\overrightarrow{\operatorname{Tr}}^{\nu} f_{p}\right] .
\end{aligned}
$$

Proof. Corollary 9.11 assures us that all of the first order traces of all four types exist and agree. By (9.18) of Proposition 9.4 and Corollary 9.6 we know that $\operatorname{Tr}^{\nu} f_{p}$ has the orthogonal expansion

$$
\begin{aligned}
\operatorname{Tr}^{\nu} f_{p} & =\sum_{i_{2 \nu+1}, \ldots, i_{p}=1}^{\infty} \\
& \cdot\left(\sum_{j_{1}, \ldots, j_{\nu}=1}^{\infty} a_{j_{1}, j_{1}, \ldots, j_{\nu}, j_{\nu}, i_{2 \nu+1}, \ldots, i_{p}}\right)^{2} \phi_{i_{2 \nu+1}} \otimes \cdots \otimes \phi_{i_{p}} .
\end{aligned}
$$

But the coefficients in the expansion (9.58) are in $l_{1}$ because of our assumption that $\left(a_{i_{1}}, \ldots, i_{p}\right) \in l_{1}$ and the inequalities

$$
\begin{aligned}
& \sum_{i_{2 v+1}, \ldots, i_{p}=1}^{\infty}\left|\sum_{j_{1}, \ldots, j_{\nu}=1}^{\infty} a_{j_{1}, j_{1}, \ldots, j_{\nu}, j_{\nu}, i_{2 \nu+1}, \ldots, i_{p}}\right| \\
& \leq \sum_{i_{2 \nu+1}, \ldots, i_{p}=1}^{\infty} \sum_{j_{1}, \ldots, j_{\nu}=1}^{\infty} \mid a_{j_{1}, j_{1}, \ldots, j_{\nu}, j_{\nu}, i_{2 \nu+1}, \ldots, i_{p}}<\infty .
\end{aligned}
$$

Applying Corollary 9.11 again but this time to $\operatorname{Tr}^{\nu} f_{p}$, we now know that all the $k$-traces of all four types of $\operatorname{Tr}^{\nu} f_{p}, 0 \leq k \leq[(p-2 \nu) / 2]$, exist and agree. The consistency of the first and second order traces of all four types follows from Proposition 9.3 and Corollaries 9.3, 9.4 and 9.9.

\section{Extension to ABSTRACT WienER SPACE}

The principal results of the previous sections remain true for an abstract Wiener space (AWS), $(H, B, P)$. We will briefly indicate some of the main features of this extension.

Let $B$ be a separable Banach space, $H$ a real separable Hilbert space densely imbedded in $B$ such that the injection is continuous. We then have the triplet $B^{*} \subset H^{*}=H \subset B$ where $*$ denotes the dual and $H^{*}$ is identified with $H . P$ is a centered Gaussian measure on $\mathscr{B}(B)$, the $\sigma$-field of Borel sets of $B$ with covariance

$$
E\left(\langle y, x\rangle\left\langle y^{\prime}, x\right\rangle\right)=\left(y, y^{\prime}\right), \quad y, y^{\prime} \in B^{*} \subset H^{*}=H .
$$


Here $\langle$,$\rangle is the \left(B^{*}, B\right)$-canonical form and $($,$) denotes the inner product$ in $H$. See [13] for further details and references.

The main results of the preceding sections can now be generalized for an arbitrary AWS. The generalization is of interest since it applies to a wide class of Gaussian processes including multiparameter Wiener processes and more general Gaussian random fields.

Every $u \in L^{2}$ has the well-known chaos (or Wiener chaos) expansion

$$
u=\sum_{m=0}^{\infty} u_{m}=\sum_{m=0}^{\infty} J_{m}\left(F_{m}\right)
$$

where $F_{m} \in H^{\otimes m}$ and $u_{m}=J_{m}\left(F_{m}\right)$ is the $m$ th order chaos expansion in terms of Fourier-Hermite functionals (see [11]). (10.2) may also be expressed as

$$
L^{2}=\sum_{m=0}^{\infty} \oplus \mathscr{H}_{m}
$$

the RHS being an orthogonal decomposition and $\mathscr{H}_{m}$, the $m$ th order homogeneous chaos. The relation $\mathscr{H}_{m} \rightarrow H^{\otimes m}$ is an isometry given by $u_{m} \rightarrow \widetilde{F}_{m}$ where $\widetilde{F}_{m}=\mathscr{P}_{m} F_{m}$ and $\mathscr{P}_{m}$ is the orthogonal projection from $H^{\otimes m}$ onto $H^{\hat{\otimes} m}$. Furthermore we have

$$
\left\langle J_{m}\left(F_{m}\right), J_{n}\left(F_{n}\right)\right\rangle=m !\left|\widetilde{F}_{m}\right|^{2} \quad \text { if } m=n, \quad \text { and } \quad=0 \text { if } m \neq n .
$$

The definitions of the different types of $k$-traces given in $\S \S 3$ and 9 apply also to any $f_{p} \in H^{\hat{\otimes} p}$, the $p$-fold symmetric tensor product of $H$.

The definitions of first and second order traces is the same as that given in $\S 9$. Corollaries $9.3,9.4,9.9,9.10$, and 9.11 also hold good without any change.

Taking $H=L^{2}\left(\mathbb{R}^{p}\right)$ we note that the proof of Theorem 9.3 carries over, with minor changes, to the case when $f_{p} \in L^{2}\left(\mathbb{R}^{p}\right)$. We are then able to prove the following result whose interest lies in the fact that it connects the existence of $k$-traces with smoothness properties of functions.

Theorem 10.1. Let $f_{p}$ be a symmetric function belonging to $\mathscr{S}\left(\mathbb{R}^{d}\right)$, the space of rapidly decreasing functions. Then all four $k$-traces of $f_{p}$ exists and are equal.

Proof. The operator $A=-d^{2} / d t^{2}+1+t^{2}$ with dense domain in $L^{2}(\mathbb{R})$ has the normalized Hermite functions $\left\{\varphi_{n}\right\}$ as its eigenvalues. It is a well-known fact that

$$
\begin{aligned}
& \mathscr{S}\left(\mathbb{R}^{d}\right)=\left\{f_{p} \in L^{2}\left(\mathbb{R}^{p}\right): \sum_{i_{1}, \ldots, i_{p}}\left(\lambda_{i_{1}} \cdots \lambda_{i_{p}}\right)^{2 r}\left(f_{p}, \varphi_{i_{1}} \otimes \cdots \otimes \varphi_{i_{p}}\right)^{2}<\infty\right. \\
& \forall r=0,1, \ldots\}
\end{aligned}
$$

where we write $\lambda_{j}=2 j+2, j \in \mathbb{N}_{0}$. (See Yuh-Jia Lee, Analytic version of test functionals, Fourier transform and a characterization of measures in white noise calculus, J. Funct. Anal. 100 (1991), 359-380.) 
Hence the terms $\left(\lambda_{i_{1}} \cdots \lambda_{i_{p}}\right)^{2 r}\left(f_{p}, \varphi_{i_{1}} \otimes \cdots \otimes \varphi_{i_{p}}\right)^{2}$ are bounded by a constant $K_{p}^{2}$, say. It follows that

$$
\sum_{i_{1} \cdots i_{p}}\left|\left(f_{p}, \varphi_{i_{1}} \otimes \cdots \otimes \varphi_{i_{p}}\right)\right| \leq \sum_{i_{1} \ldots i_{p}} \frac{K_{p}}{\left(\lambda_{i_{1}} \cdots \lambda_{i_{p}}\right)^{2 r}}=K_{p}\left\{\sum_{n=0}^{\infty} \frac{1}{(2 n+2)^{2 r}}\right\}^{p}<\infty
$$

for $2 r>1$. The result follows from the modified version of Theorem 9.3 and Corollary 9.11 .

Let $\psi_{p}(h):=\left(f_{p}, h^{\otimes p}\right), h \in H, f_{p} \in H^{\hat{\otimes} p}$ be a symmetric $p$-linear form on $H$. It has been shown in [13] that if $(H, B, P)$ is an AWS, the probability space $(B, \mathscr{B}(B), P)$ can be taken (among other choices) as a representation space for $(H, \mathscr{C}, \mu)$ where $\mathscr{C}$ is the family of finite dimensional Borel cylinder sets in $H$ and $\mu$ is the (finitely additive) canonical Gauss measure on $H$. In the terminology of [13], a representation of $\mu$ is given by $(L, P)$ where for $h \in H$,

$$
L(h)(x):=\sum_{j=1}^{\infty}\left(h, e_{j}\right) f_{j}[x], \quad(x \in B)
$$

if the series converges and $=0$ otherwise. Here $\left(e_{j}\right) \in \gamma^{*}\left(B^{*}\right)$ is a fixed CONS in $H$ and $f_{j} \in B^{*}$ is such that $e_{j}=\gamma^{*}\left(f_{j}\right)$. The map $\gamma^{*}$ is the adjoint of the imbedding $\gamma$ of $H$ into $B$.

With this set up, the definition of an $\mathscr{L}^{2}$ lifting of $\psi_{p}$ is defined as in $\S 2$ and is denoted by $R\left[\psi_{p}\right]$. If the latter exists $\psi_{p}$ is called an accessible random variable on $H$.

The proofs of the following basic results are exactly as in Theorems 5.1 and 6.1 (for $\sigma=1$ ).

Theorem 10.2. R $\psi_{p}$ exists if and only if $\overrightarrow{\mathrm{Tr}}^{k} f_{p}$ exists for $0 \leq k \leq[p / 2]$ and then

$$
R \psi_{p}=\sum_{k=0}^{[p / 2]} C_{p, k} J_{p-2 k}\left(\overrightarrow{\operatorname{Tr}}^{k} f_{p}\right)
$$

where $C_{p, k}=p ! /(p-2 k) ! 2^{k} k !$.

Theorem 10.3. Let $f_{p} \in H^{\hat{\otimes} p}$ and let $\psi_{p}=\psi_{p}\left(f_{p}\right)$ be the associated form on $H$. Suppose that $f_{p}$ has all its first and second order traces and that they are consistent. Then

$$
J_{p}\left(f_{p}\right)=\sum_{k=0}^{[p / 2]}(-1)^{k} C_{p, k} R\left[\psi_{p-2 k}\left(\overrightarrow{\operatorname{Tr}}^{k} f_{p}\right)\right] .
$$

In a recent paper [19] Sugita has obtained the RHS of (10.4) as an expression for what he calls the multiple Stratonovich integral and denotes by $\delta^{0 p} f_{p}$. He also obtains formula (10.5) with the lifting on the RHS of (10.5) replaced by $\delta^{0 p-2 k}\left(\right.$ Trace $\left.^{k} f_{p}\right)$. However, Sugita's traces are iterated $k$-traces whereas ours are limiting $k$-traces and the two coincide under the assumption that the $k$ traces of $f_{p}$ exist. Moreover by our Theorem 10.2 the existence of limiting $k$-traces is a necessary and sufficient condition for the formula (10.4) to hold. 


\section{ACKNOWLEDGMENTS}

The first author wishes to thank the Center for Stochastic Processes for support and hospitality during his visits from November, 1987-April, 1988 and in May and June of 1989 and the AFOSR for support through a grant to the center. He also wishes to acknowledge the support of the University of Nebraska-Lincoln during his sabbatical leave in 1987-1988. The second author's research was supported by the Air Force Office of Scientific Research Contract No. F49620 85C 0144.

\section{REFERENCES}

1. S. A. Albeverio and R. J. Hoegh-Krohn, Mathematical theory of Feynman path integrals, Lecture Notes in Math., vol. 523, Springer-Verlag, Berlin, 1976.

2. A. V. Balakrishnan, On the approximation of Itô integrals using band-limited processes, Tech. Rep. UCLA-ENG-7342, UCLA School of Engineering and Applied Science, 1973.

3. R. H. Cameron and D. A. Storvick, Some Banach algebras of analytic Feynman integrable functionals, Analytic Functions, Kozubnik, 1979, Lecture Notes in Math., vol. 798, Springer-Verlag, Berlin, 1980, pp. 18-67.

4. E. Hille and R. S. Phillips, Functional analysis and semigroups, Amer. Math. Soc. Colloq. Publ., vol. 31, Providence, R.I., 1957.

5. Y. Z. Hu and P. A. Meyer, Chaos de Wiener et integrale de Feynman, Séminaire de Probabilitiés XXII, Université de Strasbourg, 1987, Lecture Notes in Math., vol. 1321, SpringerVerlag, Berlin, 1988, pp. 51-71.

6. K. Itô, Multiple Wiener integrals, J. Math. Soc. Japan 3 (1951), 157-169.

7. G. W. Johnson, The equivalence of two approaches to the Feynman integral, J. Math. Phys. 23 (1982), 2090-2096.

8. G. W. Johnson and G. Kallianpur, Some remarks on Hu and Meyer's paper and infinite dimensional calculus on finitely additive canonical Hilbert space, Theory Probab. Appl. 34 (1989), 742-752.

9. G. W. Johnson and D. L. Skoug, Scale invariant measurability in Wiener space, Pacific J. Math. 83 (1979), 157-176.

10. __ Notes on the Feynman integral . III, Pacific J. Math. 105 (1983), 321-358.

11. G. Kallianpur, Stochastic filtering theory, Appl. Math., vol. 13, Springer-Verlag, Berlin, 1980.

12. G. Kallianpur, D. Kannan, and R. L. Karandikar, Analytic and sequential Feynman integrals on abstract Wiener and Hilbert spaces, and a Cameron-Martin formula, Ann. Inst. Henri Poincaré 21 (1985), 323-361.

13. G. Kallianpur and R. L. Karandikar, White noise theory of prediction, filtering and smoothing, Stochastic Monographs no. 3, Gordon and Breach, New York, 1988.

14. G. Kallianpur and A. S. Ustunel, Distributions, Feynman integrals and measures on abstract Wiener spaces, Stochastic Analysis and Related Topics, (H. Körezlioglu and A. S. Ustunel, eds.) Birkhäuser, Boston, Mass., 1992, pp. 237-284.

15. J. Kelley, General topology, Van Nostrand, 1955.

16. J. Lindenstrass and L. Tzafriri, Classical Banach spaces. I, Sequence spaces, Ergebnisse Math. Grenzgeb., vol. 92, Springer-Verlag, New York, 1977.

17. J. Rosinski, On stochastic integration by series of Wiener functionals, Appl. Math. Optim. 19 (1989), 137-155.

18. A. V. Skorokhod, On a generalization of a stochastic integral, Theory Probab. Appl. 20 (1975), 219-233.

19. H. Sugita, Hu-Meyer's multiple Stratonovich integral and essential continuity of multiple Wiener integral, preprint. 


\section{FURTHER REFERENCES (ADDED IN PROOF)}

[A] G. W. Johnson and G. Kallianpur, Multiple Wiener integrals on abstract Wiener spaces and liftings of p-linear forms, White Noise Analysis, (Bielefeld Conference, T. Hida, et al., eds.), World Scientific, Singapore, 1990, pp. 208-219.

[B] _ The analytic Feynman integral of the natural extension of pth homogeneous chaos, Measure Theory, Oberwolfach, (D. Kölzow, et. al., eds.), Rend. Circ. Mat. Palermo (2) Suppl. 28 (1992), 181-199.

[C] _ Remarks on the existence of $k$-traces (submitted).

[D] G. Kallianpur, Traces, natural extensions and Feynman distribution, Gaussian Random Fields, (Nagoya Conference, K. Itô and T. Hida, eds.), World Scientific, Singapore, 1991, pp. 14-27.

[E] G. Kallianpur and R. L. Karandikar, Nonlinear transformations of the canonical Gauss measure on Hilbert space and absolute continuity, Acta Math. Appl. (to appear).

Department of Mathematics and Statistics, University of Nebraska, Lincoln, NeBRASKA $68588-0323$

Department of Statistics, University of North Carolina, Chapel Hill, North CarOLINA 27599-3260 